

\section{Editor-in-chief:}

Prof. Arnold van Huis, Wageningen University, the Netherlands

\section{Associate editors:}

Prof. Eraldo M. Costa-Neto, Universidade Estadual de Feira de Santana, Brazil; Dr Mark Finke, Mark Finke LLC, USA; Dr Arnout Fischer, Wageningen University, the Netherlands; Dr Laura Gasco, University of Turin, Italy; Dr John N. Kinyuru, Jomo Kenyatta University of Agriculture \& Technology, Kenya; Prof. Santos Rojo, University of Alicante, Spain; Dr Nanna Roos, University of Copenhagen, Denmark; Dr Birgit Rumpold, Technische Universität Berlin, Germany; Prof. Jeffery K. Tomberlin, Texas A\&M University, USA; Prof. Leen Van Campenhout, KU Leuven, Belgium

\section{Editorial board:}

Prof. Jérôme Casas, University of Tours, France; Dr Adrian Charlton, FERA, United Kingdom; Prof. Xiaoming Chen, Research Institute of Resources Insects, China; Dr Florence Dunkel, Montana State University, USA; Patrick Durst, Forestry and natural resources consultancy, Thailand; Prof. Jørgen Eilenberg, University of Copenhagen, Denmark; Dr Sunday Ekesi, icipe, Kenya; Prof. Kokoete Ekpo, Federal University Otuoke, Nigeria; Prof. Ying Feng, Research Institute of Resources Insects, China; Prof. Lynn Frewer, Newcastle University, United Kingdom; Prof. Richou Han, Guangdong Academy of Sciences, China; Dr Yupa Hanboonsong, Khon Kaen University, Thailand; Dr Marc Kenis, CABI, Switzerland; Dr Catriona Lakemond, Wageningen University, the Netherlands; Prof. Harinder Makkar, University of Hohenheim, Germany; Dr José Manuel Pino Moreno, Universidad Nacional Autónoma de México, Mexico; Prof. Benno Meyer-Rochow, Oulu University, Finland; Andong University, South Korea; Prof. Kenichi Nonaka, Rikkyo University, Japan; Dr Søren Bøye Olsen, University of Copenhagen, Denmark; Prof. Maurizio G. Paoletti, University of Padova, Italy; Prof. John Schneider, Mississippi State University, USA; Dr Oliver Schlüter, Leibniz Institute for Agricultural Engineering Potsdam-Bornim, Germany; Prof. Joop van Loon, Wageningen University, the Netherlands; Dr Teun Veldkamp, EAAP Commission on Insects / Wageningen Livestock Research, the Netherlands; Prof. Wim Verbeke, Ghent University, Belgium; Dr Jintana Yhoung-Aree, Institute of Nutrition, Mahidol University, Thailand; Prof. Jose Jacobo Zubcoff Vallejo, University of Alicante, Spain

\section{Publication information}

Journal of Insects as Food and Feed

ISSN 2352-4588 (online edition)

Subscription to 'Journal of Insects as Food and Feed' (4 issues a year) is either on institutional (campus) basis or on personal basis. Subscriptions can be online only. Prices are available upon request from the publisher or from the journal's website (www.wageningenacademic.com/jiff). Subscriptions are accepted on a prepaid basis only and are entered on a calendar year basis. Subscriptions will be renewed automatically unless a notification of cancellation has been received before the $1^{\text {st }}$ of December before the start of the new subscription year.

Further information about the journal is available through the website www.wageningenacademic.com/jiff.

\section{Paper submission}

Manuscripts should be submitted via our online manuscript submission site, www.editorialmanager.com/jiff. Full instructions for electronic submission, as well as the guideline for authors are directly available from this site or from www.wageningenacademic.com/jiff.

\section{Internet access}

The online edition is available at www.wageningenacademic.com/jiff with free abstracts and keywords. A RIS alert for new online content is available as well.

\section{Editorial office}

jiff@wageningenacademic.com

\section{Orders and claims}

P.O. Box 220

6700 AE Wageningen

The Netherlands

jiff_cr@wageningenacademic.com

Tel: +31 317476516

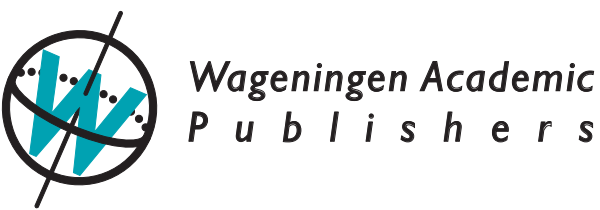




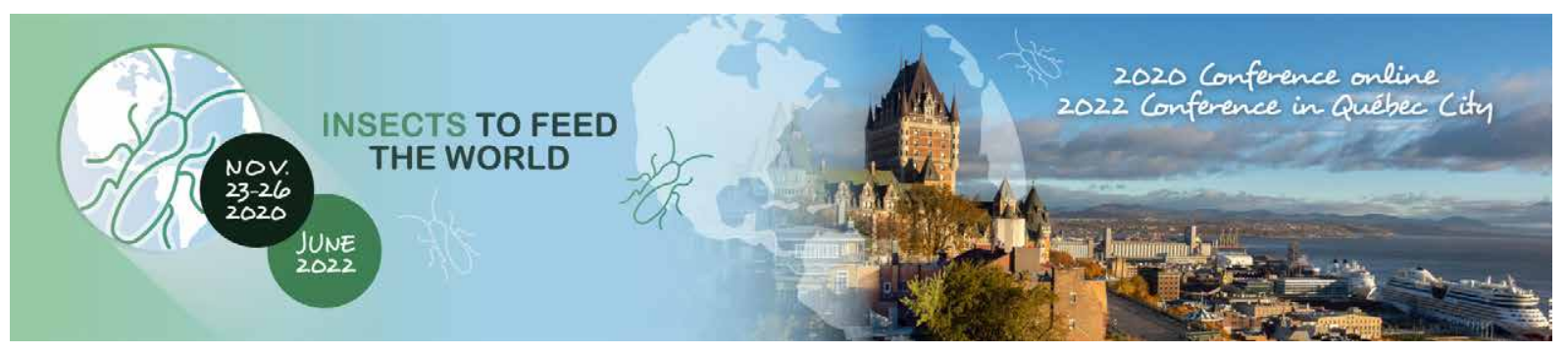

\title{
Insects to Feed the World 2020 Virtual Conference
}

\author{
November 23-26, 2020 \\ This supplement of Journal of Insects as Food and Feed can be found at \\ https://www.WageningenAcademic.com/IFW2020.
}


- Lucie Beaulieu, Université Laval

\section{Local organising committee}

- Marie-Hélène Deschamps, Université Laval

- Alain Doyen, Université Laval

- Louise Hénault-Éthier, David Suzuki Foundation

- Charles Lavigne, Quebec's Agrifood Innovation Centre

- Anand Naraine, Industry showcase Coordinator

- Nicolas Turgeon, Centre de recherche industrielle du Québec

- Grant W. Vandenberg, Université Laval

\section{International advisory committee}

- Arnold van Huis, Wageningen University \& Research, the Netherlands

- Jeffery K. Tomberlin, Texas A\&M University, USA

- Longyu Zheng, Huazhong Agricultural University, China

\section{Scientific committee}

- Lucie Beaulieu, Universite Laval

- Guido Bosch, Wageningen University

- Diana Bogueva, Curtin University

- Annette Bruun Jensen, University of Copenhagen

- Marie-Hélène Deschamps, Université Laval

- David Deruytter, Inagro

- Marcel Dicke, Wageningen University

- Alain Doyen, Université Laval

- Sunday Ekesi, International Centre of Insect Physiology and Ecology (icipe)

- Jørgen Eilenberg, Department of Plant and Environmental Sciences

- Lotte Frooninckx, Thomas More University of Applied Sciences

- Ying Feng, Research Institute of Resource Insects

- Laura Gasco, University of Turin

- Mickey Gjerris, University of Copenhagen

- Moritz Gold, ETH Zurich

- Christina Hartmann, ETH Zürich Dep. of Environmental Systems Science

- Yupa Hanboonsong, Khon Kaen University

- Louise Henault-Éthier, Université Laval/Tricycle

- Segenet Kelemu, International Centre of Insect Physiology and Ecology (icipe)

- John Ndung'u Kinyuru

- Julie Lesnik, Wayne State University

- Marie-Pierre Létourneau-Montminy, FSAA Université Laval

- Alexandre Mathys, ETH Zurich
- Dennis Oonincx, Wageningen University

- Rocio Ponce Reyes, Commonwealth Scientific and Industrial Research Organisation | CSIRO

- Birgit Rumpold, Technische Universität Berlin

- Chris Sandrock, Research Institute of Organic Agriculture, Switzerland

- Linda Saucier, Université Laval

- Eric Schmitt, Protix

- Matan Shelomi, Department of Entomology, Max Planck Institute of Chemical Ecology

- Sergiy Smetana, German Institute of Food Technologies

- Valerie Stull, University of Wisconsin-Madison Global Health Institute

- Gianluca Tettamanti, University Insubria

- Jeffery K. Tomberlin, Texas A\&M University

- Leen Van Campenhout, KU Leuven

- Joop van Loon, Wageningen University

- Arnold van Huis, Wageningen University

- Grant W. Vandenberg, Université Laval

- Teun Veldkamp, Wageningen University

- Andreas Vilcinskas, LOEWE Center for Insect Biotechnology \& Bioresources

- Jibin Zhang, College of Life Science \& Technology, Huazhong Agricultural University

- Longyu Zheng, Huazhong Agricultural University

- Chris Zurbrugg, Eawag: Swiss Federal Institute of Aquatic Science and Technology 


\section{Progress in using insects for food and feed}

\section{A. van Huis \\ Emeritus professor, Laboratory of Entomology, Droevendaalsesteeg 1, 6708 PB Wageningen, the Netherlands; arnold.vanhuis@wur.nl}

During the last five years the number of articles dealing with edible insects increased exponentially. The attention shifted from harvesting insects in the tropics to farming insects all over the world for food and feed applications. The black soldier fly as feed is often targeted as they can play a role in recycling many organic waste streams of low economic value and transforming them in high value proteins and fats. Microbial communities and their relationship with insects deserve full attention as it may help in the conversion of low-quality organic side streams. The nutritional values vary between more than 2,000 species of insects available and can be tailored by using different diets and environmental conditions. Health benefits relate to insects having the largest anti-microbial peptide reservoir of all animals. The exoskeleton of insects, chitin and its deacylated form chitosan, may serve as prebiotic, both in humans and in animals. It also seems to improve plant growth and to activate plant defence. In addition, the antioxidant capacity of insect extracts looks promising. Other bio-active compounds need further to be explored. Other technological applications for fat are bio lubricants, biodiesel, cosmetics and butter replacements in bakery products. Proteins can be employed as a base for bioplastics used for agricultural purposes. The environmental impact of producing insects compares favourably to that of producing meat or the various alternative proteins used as meat substitutes. However, this as well as the profitability depend very much on the availability, quality and price of substrates used for rearing the insects. A new area is that of genetic improvement for which much progress can be expected due to the short life cycle of insects, e.g. can we select strains fit for specific waste streams? A main challenge for insects incorporated in food is to go from the occasional snack to staple foods. Food safety relate to chemical and biological contaminants and when organic side streams are used, caution is required, although some of these contaminants are reduced in the insect gut. Allergenicity is an issue when people are sensitive to closely related species, such as crustacea or house dust mites. Food safety needs full attention as it will determine the easing of legislation, which in turn will increase its potential to become a major industry. Consumers are becoming more and more aware that insects as food are a viable option, but strategies need to be finetuned to overcome the reluctance of eating insects. This means providing information about the sustainability and processing the insects disguised in familiar products, that are safe and appetising. The sector can only progress when the insect industry, the academic world, governmental organisations and public society closely cooperate. 


\title{
From research to economics: potential paths forward in the insects as food and feed industry
}

\author{
J.K. Tomberlin \\ Department of Entomology Texas A\&M University College Station, Texas, USA; jktomberlin@tamu.edu
}

Research drives economic opportunity. While such a statement may be viewed as a disconnect due to results generated from research translating into applications often being delayed. Given the insects as food and feed sector is relatively in its infancy, opportunities exist to set the stage for more efficient and timely translation of such mechanisms into relevant actions. Developing such a concept relies on four factors, which will be discussed: (1) continued efforts on basic research; (2) scale; (3) economics; and (4) development of a global economic model to project futures as related to production. Translating results across scale is not linear in most cases, which can lead to inaccuracies as related to economic-related projections. Therefore, conducting studies across different scales (e.g. experiment design as related to pan size, replicates, location, season) are crucial for understanding intra- and inter-facility (i.e. company) variation. By understanding and regulating the variation associated with production, a potential commodity group could evolve that projects futures on the production of select insects as food and feed- similar to other commodity groups, such as wheat or maize. However, efforts to bolster the industry as a commodity will need to be linked with other disciplines, such as economics, so the calculus for estimating the futures as related to production, quality, and value and be reliably determined. In the end, each of these paths is reliant on continued efforts to understand the basic nature of each insect being mass produced. Therefore, the likelihood of expansion, diversification, and solidified establishment of the insects as food and feed industry is reliant on continued research efforts to understand the 'why' and 'how' research questions.

\section{Growing a better future for our industry by harvesting the painful lessons of our past}

\author{
M. Ashour \\ Aspire Food Group, 6231 E Stassney Ln, Austin, TX 78744, USA; ma@aspirefg.com
}

The prospects have never been more exciting and the future brighter for the insect agriculture industry. New, sophisticated consumer brands are emerging. Large retailers and food companies are investing capital, brand equity, and resources to accelerate and adopt ingredients from insects. A number of insect agriculture companies in North America and Europe, including Aspire Food Group, are undertaking significant projects to build commercial production and processing facilities to deliver on the promise of efficiency at scale. As our industry scales, the magnitude - and impact - of our collective business decisions will be magnified. This means successes will be far more rewarding, and failures far more consequential. As pioneers, we embrace the unchartered waters ahead, but we do so with the many humbling lessons of our past. In this keynote presentation, the author shares candid reflections on nearly a decade of experiences - and failures - operating a vertically-integrated company in this industry. The purpose of the talk is to inspire existing and aspiring insect agriculture entrepreneurs and non-profit executives to avoid potential pitfalls in this exciting growth chapter of our industry. 


\title{
Fermentation technology in the insect industry
}

\section{Van Campenhout}

KU Leuven, Department of Microbial and Molecular Systems (M²S), Lab4Food, Campus Geel, Kleinhoefstraat 4, 2440 Geel, Belgium; leen.vancampenhout@kuleuven.be

In a fermentation process, micro-organisms generally are held in a fermentation substrate under certain environmental conditions to introduce desired changes in the substrate. The objective can be the production of microbial cells, the production of enzymes or metabolites, or the process can have the aim to transform one specific component into another. There are ample and worldwide examples of fermentation processes in the food and in other industries. Also for the insect industry, fermentation technology can provide a number of opportunities. This presentation will focus in the first place on fermentation of rearing substrates for insects and in the second place on fermentation of the harvested insects. Substrates can be fermented, either as a liquid state fermentation or as a solid state fermentation or silage process to improve the shelf life of the substrates, in particular when the supply of substrate to the rearing facility is higher that the feeding rate, and to increase the digestibility and microbial safety of the substrates. While examples of this practice can be found in industry, e.g. the fermentation of food waste or potato peels, the impact of fermentation of the substrate on insect growth is hardly documented in literature. More public data with respect to the impact of e.g. the $\mathrm{pH}$ reduction by fermentation and the altered (micro)structure of the substrate on insect growth and microbiology are needed for the sector. Within our research group Lab4Food, we currently study the fermentation of substrates for rearing of black soldier fly larvae (BSFL) by lactic acid bacteria isolated from the BSFL gut. Possibly, one or more isolates could first serve as starter culture to ferment the substrate for preservation and digestibility improvement, and then later, when the starter has colonised the substrate, it can behave as a probiotic during rearing and enhance larval growth. In addition to being useful for rearing substrates, fermentation potentially brings added value to harvested insects. Similar as to shrimp paste, it is possible to pulverise insects, add a starter culture and ferment the paste. Literature on this practice is extremely scarce, but research at Lab4Food pointed out that blanched mealworms cannot be fermented with commercial meat starter cultures as a whole, but it is possible after fragmentation into paste, as indicated by the rapid $\mathrm{pH}$ decrease. Fermentation of mealworm paste decreased protein content and solubility (as they were consumed by the starters), but it increased shelf life of the paste in contrast to the use of traditional meat preservatives. Experience with BSFL fermentation using the same protocols as for mealworms is currently being built up and will be discussed.

\section{Immunity of farmed insects: basic and applied aspects}

\author{
A. Vilcinskas ${ }^{1,2}$ \\ ${ }^{1}$ Fraunhofer Institute for Insect Biotechnology and Bioresources, Department of Bioresources, Ohlebergsweg 12, 35392 \\ Giessen, Germany; ${ }^{2}$ Institute for Insect Biotechnology, Justus-Liebig University of Giessen, Heinrich-Buff-Ring 26-32, 35392 \\ Giessen, Germany; andreas.vilcinskas@ime.fraunhofer.de
}

The farming of edible insects has emerged as a promising alternative strategy for the production of protein-rich food and feed. The industrial production of insect-derived protein is more cost-effective and energy-efficient than livestock farming or aquaculture, and results in a lower ecological footprint. However, mass rearing of farmed insects and microbial contamination of their diets promote the spread pathogens and parasites. The use of antibiotics in livestock farming has been recognized as a major reason for the increasing prevalence of antibiotic-resistant human pathogens. The presentation aims to expand the advantages of insect farming versus livestock farming beyond economic arguments and a beneficial ecological footprint to include options enabling industrial protein production without the reliance on antibiotics. Insects possess a potent innate immune system which encompasses the synthesis of a broad spectrum of antimicrobial peptides. These immunity-related effector molecules contribute to the control of the insect gut microbiota, the sanitation of the gut prior to pupation and to self-medication. The presentation will briefly summarize our current knowledge about innate immunity in farmed insects or closely related model insects, and focus on features that are promising for the development of strategies to prevent the outbreak of infections without the application of antibiotics thereby achieving beneficial carry-over effects for the consumer. Recent insights into trans-generational immune-priming and nutritional immunology of farmed insects such as the Black soldier fly Hermetia illucens and the mealworm Tenebrio molitor will be highlighted. These findings are important to understand the role of the insect gut microbiota and its manipulation by addition of beneficial microbes to the diet. The potential use of probiotics to strengthen the pathogen resistance of farmed insects will be discussed. 


\title{
Insects as food and feed source: research trends in Africa
}

\author{
M.C. Tanga, X. Cheseto, P.J. Egonyu, S. Kelemu, B. Torto, S. Sevgan and S. Ekesi \\ International Centre of Insect Physiology and Ecology, Research, P.O. Box 30772, 00100 Nairobi, Kenya; ctanga@icipe.org
}

Globally, dietary deficiencies in protein, iron (Fe), Zinc (Zn) and folic acids are widespread, causing serious health problems such as anaemia, poor pregnancy outcomes, increased risk of morbidity and mortality, stunted growth, and impaired physical and cognitive development. Young children and pregnant women are the most risk group, especially in resource poor communities. Also, most of the available and accessible nutrient-rich food sources such as fish and soybean are imported and directed towards animal feed production. Therefore, the need to search for urgent eco-friendly alternatives becomes crucial. Edible insects are promising potential and sustainable alternative nutrient source for human and animal nutrition. Entomophagy is remarkably experiencing steady increase in sub-Saharan African countries where insects are widely accepted. This study provides in-depth information on inventory of edible insects in Africa and their protein, fat, amino acids, flavonoids, minerals (Fe, Zn), vitamins, chitosan, and digestibility values. Our results showed that over 552 insect species were consumed as delicacy in $75 \%$ of the countries in Africa. Over $80 \%$ of the species had crude protein contents that surpasses that of most plant sources but comparable to that of animal sources. The levels of Fe and Zn present in 8 edible insect species was 3-6fold and 2-4-fold higher, respectively, than plant and animal-based food sources. Insect oils were much richer flavonoids, vitamin $\mathrm{E}$ and omega-3/6 fatty acids. Insects had well-based levels of essential amino acids with 2-3 folds increase in lysine and methionine, which are highly limited in cereal-and-legume based food products. Folic acid levels of two cricket species (Scapsipedus icipe and Gryllus bimaculatus) was found to be 4-5-fold higher compared to that of plant and animal-based food sources. Chitosan extracts from crickets significantly inhibited clinical pathogens in vitro. Furthermore, fish, poultry and pig fed insect-based feeds had significantly improved growth performance, egg production, egg quality, carcass quality and profitability than conventional fishmeal-based feeds. The impact of insect frass fertilizer on the growth and yields of vegetable crops and maize was significantly higher compared to existing commercial organic and synthetic fertilizers. These findings have enabled the development of standards that allows the use of insects for food and feed in Kenya and Uganda. We conclude that edible insects present unique opportunities for improving and filling the food and nutritional gap, especially for the rapidly growing undernourished populations in many resource-poor communities in Africa and beyond with a lower negative impact on the environment.

\section{Market size for insects as feed}

\section{T. Thompson}

Enterra E The Cambridge Group, Enterra: 20240 Stewart Crescent, Maple Ridge, BC V2X 0T4, Canada; tthompson@enterra.com

There has been much interest in the market size of insects for animal feeds. This report by Enterra and The Cambridge Group seeks to answer that question by examining five key markets: pet, commercial poultry, commercial aquaculture, backyard chicken and wild bird. To validate the size, point of sale data was purchased from Nielsen, the global leader in market measurement data. Also reviewed were over 100 pieces of publicly available information, along with interviews from experts from leading pet food companies such as MARS Petcare and numerous technical experts across all markets. This report identifies immediate demand of $\sim 150,000$ tonnes in 2020 for premium applications in North America and Europe with high growth rates and premium price points. Further out, additional markets come online with demand for $\sim 425,000$ tonnes. Finally, this report illustrates the 2.6 million tonne gap created by commercial aquaculture and commitments made by industry leaders such as Skretting to address this gap. 


\title{
Is there an opportunity for insects in mainstream commerce? Market size and consumer attitudes towards insects in pet foods
}

\author{
B. Jowett \\ Enterra 20240 Stewart Crescent, Maple Ridge, BC V2X 0T4, Canada; bjowett@enterra.com
}

There has been much interest and consternation in the market size of insects for animal feeds. Enterra collaborated with The Cambridge Group to uncover the answer to the question of 'Just how big is the market?' By examining five key markets: pet, commercial poultry, commercial aquaculture, backyard chicken and wild bird, the discovery was that the market opportunity was extensive and growing. To validate the size, point of sale data was purchased from Nielsen, the global leader in market measurement data. Also reviewed were over 100 pieces of publicly available information, along with interviews from experts from leading pet food companies such as MARS Petcare and numerous technical experts across all markets. This report identifies immediate demand of $\sim 150,000$ tonnes in 2020 for premium applications in North America and Europe with high growth rates and premium price points. Further out, additional markets come online with demand for $\sim 425,000$ tonnes. Finally, this report illustrates the 2.6 million tonne gap created by commercial aquaculture and commitments made by industry leaders such as Skretting to address this gap. Within the \$32.6 billion USD (2017) pet food market there is a premium sector that is growing fastest. Insect-based proteins are just breaking into this market and still not considered a mainstream ingredient but present a solution that consumers are looking for. Even without any education, $23 \%$ of consumers would be willing to try an insect-based protein product. This number increased to $36 \%$ with only minor education on the benefits and advantages of insect-based proteins. Education, transparency and a strong industry are key for success.

\section{Holistic sustainability assessment of global food systems - challenges, needs and tools with perspective on insects}

\author{
A. Mathys \\ ETH Zurich, Institute of Food, Nutrition and Health, Sustainable Food Processing Laboratory, Schmelzbergstrasse 9 , 8092 \\ Zurich, Switzerland; alexander.mathys@hest.ethz.ch
}

Food systems are at the heart of at least 12 of the 17 Sustainable Development Goals (SDGs). The wide scope of the SDGs calls for holistic approaches that integrate 'siloed' food sustainability assessments in order to develop solutions able to change complex food systems. Despite numerous developed solutions the problems associated with the excessive use of natural resources for food production and food waste are not solved. Such problems are considered as one of the most crucial for our global food systems and sustainable development. The alliance of novel production technologies, such as insect production for food and feed, with integrated sustainability assessment in real time and further data integration into national food systems through nutritional, environmental and social indicators could be a basis for the holistic development of more sustainable food systems. For emerging insect production systems, holistic life cycle sustainability assessment, aligned with introduced process innovations, can evaluate the suggested solutions on a multi parameter base, in terms of sustainability of improved insect production. Life cycle sustainability assessment (LCSA) in this perspective could potentially evaluate all environmental, social and economic impacts and benefits in the context of decision-making processes towards the development of sustainable products throughout their life cycle, if holistic data sets would be available. The integration of advanced sustainability assessment with nutritional properties can provide new assessments of the real value of innovative food products enabled by emerging production technologies. This allows for an improved and fair comparison between new food products against other sources, taking into consideration the respective technology readiness levels of new production processes and their economy of scale. Environmental life cycle assessment (LCA) as part of LCSA relies on attributional modelling for the estimation of the most impacting stages of insect production and on consequential modelling for the estimation of potential benefits or risks for the agrifood system. Performed environmental sustainability analyses of existing insect production processes largely correspond with other findings in the literature, indicating fresh insect biomass is almost twice more sustainable than fresh chicken meat. The combination of this data with social and economic sustainability impacts could strongly supports the global implementation of insect-based food and feed systems, but also other emerging and potentially more sustainable production concepts. 


\section{Insights into IPIFF activities and EU policy environment for the insect sector}

\section{H. Katz}

International Platform of Insects for Food and Feed (IPIFF), Avenue Adolphe Lacomblé 59, BTE 8, 1030 Brussels, Belgium; info@ipiff.org

IPIFF is an EU non-profit organisation which represents the interests of the insect production sector towards EU policy makers, European stakeholders, and citizens. Composed of 71 members, IPIFF promotes the use of insects for human consumption and insect-derived products as a top tier source of nutrients for animal feed. The associations, main mission is to promote the wider use of insects as an alternative or new source of protein for human consumption and animal feed through continuous dialogue with the European institutions as well as international organisations. Notably, IPIFF centres its activities around advocating for appropriate EU legislative frameworks to apply to insect production. IPIFF actively supports the responsible development of the insect sector though its various sector specific publications. To accomplish its mission, the association helps its members in the effective implementation of EU food \& feed safety legislations, such as through the development of shared standards and good hygiene practices. Furthermore, IPIFF aims to inform and communicate on the benefits of eating insects and their use as animal feed towards the wider public. As European association representing 71 members based in 23 countries, IPIFF has singled out education as a critical mean to foster the development of the insect sector. Furthermore, IPIFF collaborates with renowned universities and research institutes as well as with several insect producers established outside Europe: these actors have a status of associated members within the association. Registered in the EU transparency register, IPIFF is a member of various EU institutional consultative platforms established by EU public authorities (e.g. the European Commission Advisory Group on the Food Chain and Animal and Plant Health, EFSA Stakeholders' Forum). IPIFF closely collaborates with EU stakeholders associations representing and agri-food chain actors (e.g. European Farmers \& Agri-Cooperatives, Feed manufacturers, Food \& Drink industries, Veterinarians). 


\section{Insect as food \& feed/processing, conservation \& food safety of insect products}

\section{The potential of dominant micro-organisms isolated from the gut of the larvae of Hermetia illucens for microbiological control}

\section{J. De Smet ${ }^{1 *}$, E. Gorrens ${ }^{1}$, L. Van Moll ${ }^{1,2}$, P. $\operatorname{Cos}^{2}$ and L. Van Campenhout ${ }^{1}$}

${ }^{1}$ KU Leuven, Department of Microbial and Molecular Systems (M $\left.{ }^{2} S\right)$, Lab4Food, Campus Geel, Kleinhoefstraat 4, 2440 Geel, Belgium; ${ }^{2}$ Laboratory for Microbiology, Parasitology and Hygiene (LMPH), Faculty of Pharmaceutical, Biomedical and Veterinary Sciences, University of Antwerp, Universiteitsplein 1, 2610 Antwerp, Belgium; jeroen.desmet@kuleuven.be

Hermetia illucens is currently the most frequently used insect for the bioconversion of organic waste on an industrial scale. One of the reasons explaining its success is that evolution has enabled the larvae of $H$. illucens to thrive on a wide range of organic materials, ranging from rotting fruit to carcasses. While these niches can be a rich source of nutrients, they are also known to contain a high microbial load. To defend themselves these larvae express one of the highest number of antimicrobial peptides known to date for insects. Their own defence system might be further strengthened by the larval gut microbiome, because insects that occupy sophisticated ecological niches in some cases depend on their gut microbiota to prevent colonisation by invading pathogens taken up with the diet, according to evolution theory predictions. To explore if such complex interactions occur between the larvae of $H$. illucens and their gut microbes, isolates of abundant bacteria were collected from larvae fed on five nutritionally different diets, containing respectively high levels of lignin, cellulose, hemicellulose, pectin and keratin. A total of 180 isolates were obtained from plates containing the abundant strains present in the larval gut. Using RAPD, 63 unique isolates were retained from this collection and identified using MALDI-TOF. It is worth noting that almost half of the collected isolates consist of merely six unique isolates, which were identified on the different diets. In addition to findings in literature, this is another indication towards the existence of a potential core set of micro-organisms present in the larval gut. In parallel, the antimicrobial activity of all isolates against human pathogens Staphylococcus aureus, Pseudomonas aeruginosa, Salmonella enteritidis, Bacillus cereus and Candida albicans was assessed using spot tests. Several isolates were found to have antimicrobial activity against the tested micro-organisms and could be micro-organisms that help their host to control its gut microbiota, as hypothesised. Future work will focus in on these promising isolates to identify their active components and also to explore the potential use of such strains as a supplement in the rearing process to prevent colonisation by unwanted micro-organisms. 


\title{
Influence of processing methods on the techno-functional properties of flours from three edible insects
}

\author{
F.T. Fombong ${ }^{1,2,3^{*}}$, C.M. Tanga ${ }^{2}$, J. Vanden Broeck ${ }^{1}$ and J. Kinyuru ${ }^{3}$ \\ ${ }^{1}$ KU Leuven, Molecular Developmental Physiology and Signal Transduction, Division of Animal Physiology and Neurobiology, \\ Naamsestraat 59 - box 2465, 3000 Leuven, Belgium; ${ }^{2}$ International Center of Insect Physiology and Ecology (ICIPE), Plant \\ Health Theme, Insects for food and feed programme, Duduville campus, P.O. Box 30772-00100 Nairobi, Kenya; ${ }^{3}$ Jomo \\ Kenyatta University of Agriculture and Technology, Department of Food Science and Technology, P.O. Box 62000, 00200 \\ Nairobi, Kenya; tengweh.fombong@kuleuven.be
}

\begin{abstract}
Although insects are a well-known valuable alternative protein source to fish and beef in many countries, consumer acceptability remains a significant challenge, especially in the western world. However, consumer acceptance can be improved through grinding into flour and used as high-quality food ingredients. There is a lack of information on the impact of processing methods on the techno-functional properties of edible insects. The current work was aimed to investigates the influence of oven and freeze-drying methods on the techno-functionality of processed insect flours from three edible insects: Ruspolia differens, Gryllus bimaculatus and Schistocerca gregaria. Fifty percent of the dried ground insect powder was defatted using hexane, while the other portion was not defatted. Selected techno-functional properties (such as water binding, water-holding, foaming capacity and stability and fat absorption capacity) of both samples was evaluated. The acid value, iodine, saponification, and peroxide values of the extracted oils were assessed. Our results revealed that there was no significant difference in the influence of drying method on the techno-functional properties of the insect flours investigated. There was significant variation of most of the afore-mentioned physico-chemical attributes in the protein concentrate and non-defatted portions among the different insect species. Also, peroxide and acid values were significantly influenced by both drying methods and insect oil type. Irrespective of the drying method, there was a clear distinction on the quality of non-defatted insect flours and their protein concentrates which is a direct reflection of the techno-functional properties of the target insects. Our findings demonstrate that both non-defatted and defatted insect powders could serve as a convenient alternative nutrient-rich source of ingredients for inclusion in diets and other food matrices to improve on their functionality.
\end{abstract}

\section{Insect processing and insect anatomy}

\author{
C.M.M. Lakemond", D. Azzollini, D. van de Minkelis, M. Mishyna and V. Fogliano \\ Wageningen University \& Research, Food Quality and Design, P.O. Box 17, 6700 AA Wageningen, the Netherlands; \\ catriona.lakemond@wur.nl
}

The insect value chain includes processing of reared insects to make them suitable for food or feed purposes as a whole, or to obtain fractions that can be further used as ingredients. Processing is often not evaluated in relation with the insect anatomy. We present examples of how insect anatomy is important for: (1) softening whole insects using chitinase; (2) aqueous extraction of insect protein; and (3) effective biofractionation into lipid and protein. Incubation of whole Alphitobius diaperinus larvae with chitinases led to a lower hardness, crispness and fracturability upon defatting using hexane as pre-treatment. Also, the in vitro gastric protein digestibility was increased with approximately $10 \%$ after incubation with chitinase. These results are explained by the layers within the insect cuticle. The procuticle contains a strong network of chitin and protein and is covered by the epicuticle, that mainly consists of lipids, with on top a layer of unbounded wax and lipids. So, the procuticle is accessible for chitinases after addressing the epicuticle with hexane. Next to cuticular protein, other main protein classes in insects are muscle and haemolymph proteins. The haemolymph is a circulatory fluid, analogous to blood in vertebrates. In extracting protein from Tenebrio molitor larvae using an aqueous procedure at neutral $\mathrm{pH}, \mathrm{LC}-\mathrm{MS} / \mathrm{MS}$ data showed that the most abundant proteins in the water-soluble fraction were haemolymph proteins $(\sim 12 \mathrm{kDa})$ \& putative allergens (e.g. arginine kinase $\sim 30 \mathrm{kDa}$ ). The water insoluble fraction (pellet) indeed contained mainly muscle proteins (e.g. actin 30-50 kDa). Alike the three main protein classes, two different fat body types (FB) can be distinguished in insect larvae. The perivisceral FBs surround the organs in the body cavity, while the peripheral FBs are located directly under the skin. Microscopy showed that the peripheral FBs were tightly attached to muscle fibre for Hermetia illucens larvae. Aiming for a separation of lipid and protein, the exoskeleton of $H$. illucens was mechanically pressed as a first step in fractionation. After blanching $33 \%$ of the fat was found in the residue, compared to $3.3 \%$ for washing only. Lipids in the residue of blanched larvae were surrounded by protein and partly overlapping the chitinous exoskeleton, visible by an areolate surface (CSLM), suggesting that fat extraction is hampered by aggregate formation of cuticular and muscle protein including peripheral FBs. In conclusion, insect anatomy should be taken into account to develop smart and tailored processing of insects. 


\title{
Thermo-mechanical and pulsed electric field processing effects on protein quality of insect flours
}

\author{
S. Mezdour ${ }^{*}$, C. Loupiac ${ }^{2}$ and H. Mhemdi ${ }^{3}$ \\ ${ }^{1}$ Paris-Saclay Food and Bioproduct Engineering research unit (Say Food), Université Paris-Saclay, INRAE, AgroParisTech, \\ 91300 Massy, France; ${ }^{2}$ UMR Procédés Alimentaires et Microbiologiques, Equipe de Physicochimie des Aliments et du \\ Vin, AgroSup Dijon-Université de Bourgogne Franche Comté, 21000 Dijon, France; ${ }^{3}$ Sorbonne Universités, Université de \\ Technologie de Compiègne, Laboratoire Transformations Intégrées de la Matière Renouvelable, UTC/ESCOM, EA 4297 \\ TIMR, Centre de Recherche de Royallieu, CS 60319, 60203 Compiègne Cedex, France; samir.mezdour@agroparistech.fr
}

Without being the only options, it is becoming increasingly clear that insects could offer complementary and credible solutions to the problems faced by conventional protein sources, and also generate products offering a variety of micronutrients. The development of dedicated sectors will necessarily mean that account should be taken of the availability of the resource and also design of the production methods and transformation processes to be implemented. This study investigated a fractionation approach to produce protein-enriched and differently composed fractions of mealworm larvae (Tenebrio molitor), as well as Saturniidae caterpillars, in accordance with the biorefinery concept. One of the objectives was to know how the different steps of a thermo-mechanical process, including freezing, blanching, pressing, grinding, affect the quality of insect flour (colour, chemical composition, amino-acid and fatty acid profiles) as well as the physico-chemical and techno-functional properties of the protein fraction. In order to enhance the pressing kinetics and to increase the extraction yield, pulsed electric fields were applied as pre-treatment. This work allowed for the production of protein-rich insect flours whose protein content ranges from 72 to $75 \%$ (bs), with 10-14\% (bs) of lipids content and 4\% (bh) of water. The amino-acid profile of these flours meets the needs of animal and human nutrition with good protein efficiency. The production yield of $20-25 \%$ is similar to that of fishmeal production. Results showed that the application of a pre-treatment before pressing improves the extraction yield and reduces the extraction time. Pulsed electric fields seem to be a very promising technology. In fact, the application of the electrical treatment induces cell membranes permeabilization, thus facilitating the extraction stage at cold temperature and preserving product quality. These results provide insights into physico-chemical and functional properties of proteins affected by treatments. The potential of fractionation techniques for protein enrichment and delivery of a variety of differently composed fractions was demonstrated and may provide an interesting potential to optimise energy consumption during insect fractionation.

\section{Faecal sludge management using black soldier fly larvae and product safety}

\author{
D.A. Peguero ${ }^{1,2 "}$, E.T. Mutsakatira ${ }^{3}$, P.V. Tikilili ${ }^{3}$, M. Lewis ${ }^{3}$, C.S. Richards ${ }^{4}$, C.A. Buckley ${ }^{2}$ and A. Mathys ${ }^{1}$ \\ ${ }^{1}$ Laboratory of the Sustainable Food Processing, ETH Zürich, Schmelzbergstrasse 9, 8092 Zurich, Switzerland; ${ }^{2}$ Pollution \\ Research Group, University of KwaZulu-Natal, 4041 Durban, South Africa; ${ }^{3}$ The BioCycle, No 7 Road, Malukazi, \\ 4066 Malagazi, South Africa; ${ }^{4}$ AgriProtein Technologies, 1 Rochester Road, Philippi, 7750 Cape Town, South Africa; \\ daniela.peguero@hest.ethz.ch
}

Black soldier fly larvae, Hermetia illucens L. (BSFL) convert organic waste material into insect biomass, which can then be processed into a profitable and sustainable protein and fat ingredient in animal feeds. In areas where sanitation remains a challenge, the potential to valorise faecal waste material, using the BSFL technology would increase stakeholder participation in improving sanitation and encourage entrepreneurial opportunities. However, remaining barriers still exist, such as product safety. Therefore, the main objectives of this study were to evaluate BSFL development on varying ratios and combinations of differing types of human faecal waste and organic waste material, and to assess if the use of a novel non-thermal treatment technology, low energy electron beam (LEEB), could lead to safe BSFL products. The first part of the study was conducted in Durban, South Africa, where a public private partnership exists between the Ethekwini Municipality and The BioCycle, to innovatively manage faecal sludge from urine diversion toilets (UD). However, BSFL develop poorly on UD faecal sludge, due to the low nutritional quality. Therefore, the substrates tested in this study were: UD faecal sludge, ventilated improved pit latrine faecal sludge (VIP), primary sludge (PS), restaurant food waste (FW), and brewers' spent grain (SG). To evaluate this, BSFL development (mass), survivability, bioconversion and waste reduction efficiency were measured. Four-day old BSFL were reared on $250 \mathrm{~g}$ wet mass of the specified substrate at the start of the experiment, based on an assumed feeding rate of $100 \mathrm{mg}$ of substrate/larvae/day. Experiments were conducted under controlled environmental conditions with a temperature of $30 \pm 2{ }^{\circ} \mathrm{C}$ and relative humidity of $60 \pm 5 \%$ for 11 days. Results demonstrated BSFL grown on the substrate combination of $37.5 \% \mathrm{PS}, 37.5 \%$ VIP and $25 \% \mathrm{FW}(\% \mathrm{~m} / \mathrm{m})$ had a high dry bioconversion rate of $18.69 \pm 2.11 \%$ compared to $3.33 \pm 2.78 \%$ on $100 \%$ VIP. To effectively inactivate pathogens that may be present in BSFL reared on human faecal waste, BSFL were inoculated with Escherichia coli (strain K-12 MG1655) and passed through continuous LEEB at a specified dose to achieve a 3 to $5-\log 10$ inactivation. The attractiveness of using this non-thermal treatment technology is the potential in reducing microbial concentrations without product damage, in addition to extending the shelf life. 


\title{
Fat quality and phytochemical composition of reared cricket species in Kenya
}

\author{
D.K. Murugu ${ }^{1 *}$, A.N. Onyango ${ }^{1}$, A.K. Ndiritu ${ }^{1,5}$, C.M. Tanga ${ }^{2}$, I.M. Osuga ${ }^{3}$, K.K.M. Fiaboe ${ }^{2,4}$ and S. Ekesi ${ }^{2}$ \\ ${ }^{1}$ Department of Food Science and Technology, Jomo Kenyatta University of Agriculture and Technology, P.O Box 62,000- \\ 00200, Nairobi, Kenya; ${ }^{2}$ International Centre of Insect Physiology and Ecology (icipe), P.O. Box 30772-00100, Nairobi, \\ Kenya ${ }^{3}$ Department of Animal Science, Jomo Kenyatta University of Agriculture and Technology, P.O. Box 62,000-00200, \\ Nairobi, Kenya; ${ }^{4}$ The International Institute of Tropical Agriculture (IITA), B.P. 2008 (Messa); Nkolbisson; Yaoundé, \\ Cameroon; ${ }^{5}$ Department of Environmental Health, University of Kabianga, P.O. Box 2030-20200, Kericho, Kenya; \\ dorothymugane@gmail.com
}

The use of edible insects as potential alternative sources of food ingredients has been proposed as a sustainable approach towards commercialisation of edible insects' value chain in different parts of the world. In Kenya, edible crickets and grasshoppers are among the popularly promoted insects for commercialisation. This study assessed the fat quality and phytochemical composition of the newly described cricket species (Scapsipendus icipe) and Gryllus bimaculutus. Crude fat analysis and physico-chemical properties of cricket oil were determined according to standard AOAC methods. Fatty acid composition was determined using gas chromatography-mass spectrometry (GC-MS). Phytochemical analysis was done using UV-Vis spectrophotometry. Fat contents in both cricket species were comparable with a range between 31.7 and 33.5\%. G. bimaculutus was found to contain a higher amount of fat $(P=0.0005)$ indicating potential future viability as a source of oil. A total of 43 fatty acids consisting of 18 saturated fatty acids (SFA), 10 monounsaturated fatty acids (MUFA) and 5 polyunsaturated fatty acids (PUFA). The total SFA:UFA (unsaturated fatty acid) ratio recorded was 1.6: 1.4 while the fatty acid ratio (SFA:MUFA:PUFA) of 1:1:0.7 and 1:1:0.5 was noted for S. icipe and G. bimaculutus respectively. This suggests that both species could potentially provide for an adequate fat intake. PUFA proportion was higher in S. icipe (25\%) than in G. bimaculutus (18\%). Proportionately, SFA: MUFA ratio remained constant in both species but PUFA contents were most pronounced in S. icipe. Linoleic acid was the main PUFA with Scapsipedus icipe recording the higher value $(8.29 \mathrm{mg} / 100 \mathrm{~g})$ and G. bimaculutus the lower value (6.54 $\mathrm{mg} / 100$ g). Oxalates, phytates and polyphenols were detected in the two species. The values for polyphenols obtained for the two species ranged from 48 to $53 \mathrm{mg} / 100 \mathrm{~g}$. This could point to potential contribution in industrial applications as a preservative of cricket powder by inhibiting lipid oxidation. Overall, findings of this study confirm industrial viability of the two cricket species as a sustainable alternative ingredient in industrial applications through product formulations.

\section{Development of microbiome tools to improve health and safety of edible insects as components of feeds for livestock}

\author{
C.M. Tanga, A. Anyega, Y. Hamba-Tola and J.C. Paredes" \\ International Centre of Insect Physiology and Ecology (icipe), P.O. Box 30772-00100 Nairobi, Kenya; jparedes@icipe.org
}

The black soldier fly (BFS) initially known for its role as manure recycler it is now consider as a valuable food source for livestock. The larvae of BSF are rich in crude protein, fat and gross energy. They are also a rich source of micronutrients (iron and zinc) and all essential amino (such as lysine, threonine and methionine). Chitin, chitosan, and chito-oligosaccharides from BSF have immunity-enhancing effects, promote beneficial bacterial growth and inhibition of pathogenic microorganisms. Whereas BFS has an incredible potential contribution in feed security, safety concerns have been raised for its consumption due to the substrates in which it develops. Our project aim to characterise microbes associated to BSF reared in different diets with the aim of: (1) characterise and isolate its associated beneficial and detrimental microbes; (2) evaluate the transmission of potential human/livestock microbial pathogens from processed larvae through animal feeding; and thus (3) identify the best BSF larvae processing practices for inclusion in animal feed; and finally, (4) set up the bases for insect farming safety techniques. To this aims, we are currently conducting $16 \mathrm{~S}$ rRNA and ITS2 region sequencing of larval guts raised in brewery, kitchen, poultry and rabbit waste. Additionally, we plan to evaluate the transmission of microbes (bacteria and fungi) across the entire value chain; BSF larvae processing methods and swine feeding. 


\title{
Expired fish feed as a source of $n-3$ fatty acids to modulate the lipid profile of Hermetia illucens
}

\author{
D.P. Rodrigues ${ }^{1 *}$, M. Pinho ${ }^{1}$, M.R. Domingues ${ }^{2}$, J.A. Vázquez ${ }^{3}$, R. Calado ${ }^{1}$ and O.M.C.C. Ameixa ${ }^{1}$ \\ ${ }^{1}$ ECOMARE, Centre for Environmental and Marine Studies (CESAM), Department of Biology, University of Aveiro, Santiago \\ University Campus, 3810-193 Aveiro, Portugal; 2ECOMARE, Centre for Environmental and Marine Studies (CESAM), \\ Department of Chemistry, University of Aveiro, Campus de Santiago, 3810-193 Aveiro, Portugal; ${ }^{3}$ Grupo de Biotecnología \\ y Bioprocesos Marinos, Instituto de Investigaciones Marinas (IIM-CSIC), C/Eduardo Cabello, 6, CP 36208 Vigo, Galicia, \\ Spain;dmprodrigues@ua.pt
}

Palatability is an important parameter in fish feed, as it is paramount for its acceptance by cultured species. Spoilage of fish feeds is often related with lipid rancidification present in fish meal and mostly in fish oil. Under a Circular Economy framework, procedures must be introduced to prevent waste at each stage of the supply chain, to maintain materials and resources in the economy for as long as possible and, consequently, minimise the production of wastes. The capacity of the black soldier fly (BSF) Hermetia illucens to be reared under different types of substrates, including several types of wastes, as well as its ability to modulate its biochemical profile by adjusting to its feeding substrate is already well established. Successful attempts have already been performed to improve the lipid profile of $H$. illucens, by using algae or finfish as substrates, allowing the encapsulation of $\mathrm{n}-3$ polyunsaturated fatty acids (PUFA) and consequently making more feasible the inclusion of BSF meal on the formulation of aquafeeds of carnivorous marine organisms. Here we used an expired salmon aquafeed enriched with fish oil, aiming to achieve the incorporation of n-3 PUFA in H. illucens larvae, which will then be processed as BSF meal. Further, by considering different levels of fish feed in BSF larval culture, we surveyed the levels of n-3 PUFA that can be incorporated into larval tissues $\left(5^{\text {th }}, 3^{\text {rd }}\right.$ and $1^{\text {st }}$ instar) for 2,7 , and 10 days. Different percentages $(0,25,50,75$ and $100 \%)$ of expired salmon feed were blended with a control substrate (chicken feed) and used to feed the larvae of $H$. illucens. The number of days needed to achieve significant differences in H. illucens lipid profile in terms of n-3 PUFA, namely in 20:5n-3 eicosapentaenoic acid (EPA) and 22:6n-3 docosahexaenoic (DHA), was solely of 2 days with 50\% of fish feed inclusion. The maximum amount of EPA and DHA present in the larval tissues was $4.46 \%$ with $100 \%$ substitution for 10 days and $6.18 \%$ with $75 \%$ substitution and over 7 days of trial, respectively. The goal of the present study was therefore achieved, as it was possible to demonstrate that the lipid profile of $H$. illucens can be enhanced in n-3 PUFA by using an expired salmon aquafeed to feed developing larvae of BSF.

\section{Systematic studies on the antioxidant capacity of yellow mealworm larvae (Tenebrio molitor)}

\author{
N. Kröncke ${ }^{1^{*}}$, C. Keil ${ }^{2}$, F. Kulow ${ }^{2,3}$, S. Grebenteuch ${ }^{3}$, C. Kanzler ${ }^{3}$, G. Boeck ${ }^{4}$, R. Benning ${ }^{1}$ and H. Haase $^{2}$ \\ ${ }^{1}$ University of Applied Sciences Bremerhaven, Institute of Food Technology and Bioprocess Engineering, An der Karlstadt 8, \\ 27568 Bremerhaven, Germany; ${ }^{2}$ TU Berlin, Institute of Food Technology and Food Chemistry, Department Food Chemistry \\ and Toxicology, Gustav-Meyer-Allee 25, 13355 Berlin, Germany; ${ }^{3}$ TU Berlin, Institute of Food Technology and Food \\ Chemistry, Department, Food Chemistry and Analytics, Gustav-Meyer-Allee 25, 13355 Berlin, Germany; ${ }^{4}$ GloMic GmbH, \\ Krampnitzer Weg 102, 14089 Berlin, Germany; nkroencke@hs-bremerhaven.de
}

The yellow mealworm (Tenebrio molitor L., Coleoptera: Tenebrionidae) is an edible insect and will be of great importance as food and feed due to their high content of micro and macronutrients as well as their ubiquitous occurrence. Drying is an important preservation step in industrial insect production and processing. Currently, the information about the stability of functional and nutritional components in mealworms during drying is still limited. In the future, it will be necessary to find suitable strategies to restrict the oxidative spoilage of the larvae during drying procedure and post-stream processing. Concerning the problem of oxidative spoilage, the focus of the investigations is on a systematic evaluation of the antioxidative capacity of mealworm larvae depending on their drying technology. To investigate the antioxidant capacity, fresh and differently dried mealworm larvae (freeze-dried, microwave-dried, infrared-dried, oven-dried and high frequency-dried) were extracted with different solvents and evaluated for their total content of hydrophilic and lipophilic antioxidants using three antioxidant tests (TEAC, DPPH, Folin Ciocalteu). The results presented in this study show that: Fresh mealworm larvae do have slow and fast acting lipophilic and hydrophilic antioxidants. The total antioxidant capacity of hydrophilic extracted mealworm components was approximately three times higher than the lipophilic fraction. The drying process was decisive with regard to the extraction efficiency of potentially antioxidant molecules as well as the total antioxidant capacity. The highest antioxidant capacities were found in the microwave or oven-dried larvae, while the extracts obtained from the freeze-dried larvae had the lowest values. The antioxidant capacities of extracts obtained from infrared and radio frequency-dried larvae were between the aforementioned drying methods with the exception of the TEAC assay. The next step is to identify the antioxidants present in T. molitor larvae molecularly, in order to be able to develop process-optimised strategies for the best possible cultivation and processing of the mealworm larvae. 


\title{
Extraction of intracellular compounds from Tenebrio molitor by mechanical pressing and pulsed electric field treatments
}

\author{
H. Mhemdi ${ }^{1}$, C. Dridi ${ }^{1}$ and S. Mezdour ${ }^{2 *}$ \\ ${ }^{1}$ Sorbonne Universités, Université de Technologie de Compiègne, Laboratoire Transformations Intégrées de la Matière \\ Renouvelable (UTC/ESCOM, EA 4297 TIMR), Centre de Recherche de Royallieu, CS 60319, 60203 Compiègne Cedex, France; \\ ${ }^{2}$ Paris-Saclay Food and Bioproduct Engineering research unit (Say Food), Université Paris-Saclay, INRAE, AgroParisTech, \\ 91300 Massy, France; samir.mezdour@agroparistech.fr
}

Satisfying the growing demand for proteins, notably for the purpose of feeding animals, and toning down the impact of animal husbandry on the environment, while at the same responding to public demand for product quality, are major challenges for international organisations, private operators and agri-food researchers. Among several possible additional sources of proteins, the insect solution seems to be relevant and credible as a complementary option alongside other conventional ones (fish and soya). The development of an insect industry requires the development of technically reliable and economically viable transformation processes. The application of emerging technologies like pulsed electric field for the valorisation of insects seems an interesting way to explore. Unfortunately, the literature review marks the scarcity of works on this topic. The University of Technology of Compiègne (France) and AgroParisTech (France) are the first laboratories to work on this subject. Tenebrio molitor was used for investigation. Theses larvae are rich in water, proteins and lipids. The objective of the study was to fractionate the raw material in order to obtain a liquid fraction rich in oil and a solid fraction rich in proteins according to the biorefinery concept. For this purpose, mechanical expression was used for insect dewatering and defatting. In order to enhance the pressing kinetics and to increase the extraction yield, pulsed electric fields was applied as pre-treatment. Results showed that the application of a pre-treatment before pressing improves the extraction yield and reduces the extraction time. Pulsed electric fields seems to be very promising technology. In fact, the application of the electrical treatment induces cells membranes permeabilization facilitating thus the extraction step at cold temperature and preserving the product quality. The results obtained in this work will be very useful to pave the way for insects' valorisation in the biorefinery concept.

\section{Review of edible insects metabolising mycotoxins as a safe and sustainable feed/food source}

\author{
A.M. Meyer ${ }^{1 *}$, H.J. van der Fels-Klerx ${ }^{1}$, E.F. Hoek-van den Hil ${ }^{1}$ and J.J.A. van Loon ${ }^{2}$ \\ ${ }^{1}$ Wageningen Food Safety Research, Akkermaalsbos 2, 6708 WB, Wageningen, the Netherlands; ${ }^{2}$ Laboratory of Entomology, \\ Wageningen University, Droevendaalsesteeg 1, 6708 PB Wageningen, the Netherlands; amelia.meyer@wur.nl
}

Mycotoxins are highly carcinogenic and harmful to animal and human health, as well as the agricultural industry. Mycotoxins commonly occur in grains and other feed and food commodities. The occurrence of harmful concentrations of mycotoxins in agricultural commodities can be limited by agronomical practices. However, given the effects of local weather on the presence of the fungi producing mycotoxins, they cannot be avoided. Hence, in addition to the continuous attention for prevention and control strategies for mycotoxins, it is essential to find innovative and efficient ways to detoxify these harmful compounds. Continuous expansion of the global population and the resulting higher demand for crop and livestock production require an increased arable land area. The design of the insect supply chain is circular, with a low environmental footprint and efficient conversion of substrates. Certain insects can convert organic waste products into protein resourcefully, but more research is needed regarding safety issues. In previous research, the edible insects Tenebrio molitor, Alphitobius diaperinus, and Hermetia illucens were identified as capable of metabolically converting and excreting mycotoxins, such as aflatoxins and deoxynivalenol. This study aimed to review the scientific literature on the metabolization of mycotoxins by insects. The study covers various mycotoxins and several insect species fed with spiked, natural, and artificially contaminated substrates. 


\title{
Identification and characterisation of yellow mealworm's main proteins in relation to their functional properties
}

\author{
A. Gravel ${ }^{1,2^{*}}$, M. Couture ${ }^{2}$ and A. Doyen ${ }^{1}$ \\ ${ }^{1}$ Department of Food Sciences, Institute of Nutrition and Functional Foods (INAF), Université Laval, Québec, QC, G1V \\ OA6, Canada; ${ }^{2}$ Department of Biochemistry, Microbiology, and Bio-informatics, Institute of integrative biology and systems \\ (IBIS), Université Laval, Québec, QC, G1V0A6, Canada; alexia.gravel.1@ulaval.ca
}

While entomophagy is a traditional diet in more than 113 countries, this practice has completely disappeared from the lifestyle in Western countries. However, there is a growing interest in insects as human food in anticipation of the food security challenges related to the worldwide population growth. Nevertheless, food neophobia represents the major challenge which negatively affects the social acceptability for this alternative food resource. It was suggested that the integration of edible insects as food ingredients (protein concentrate or isolate) in different food formulations could enhance the consumer acceptability. In order to do so, an increased knowledge of the nature of the extracted proteins as a function of the food processing used is necessary as it is strongly related to their functional properties. Multiple defatting methods have been explored in the literature since lipid extraction is a key and crucial step during edible insect flours processing as it improves protein recovery and purity. Efficient lipid removal from the solid insect matrix remains conventional Soxhlet extraction by using hexane. However, the use of hexane could have a denaturing effect on edible insects' proteins resulting in a decrease of their functional properties. Consequently, the aim of this work is to characterise the protein profile of yellow mealworm (Tenebrio molitor) high valueadded ingredients according to the defatting process used and to evaluate its impact on protein functional properties. First, high protein content fractions were produced from untreated yellow mealworms with and without an additional hexane defatting step. Their respective protein profiles were compared using 2D-electrophoresis and proteomics. Results showed similar profiles for major edible insect proteins between hexane-defatted and non-hexane-defatted fractions. However, some specific differences were observed in terms of protein species and molecular weights which are under identification by proteomic tools. Our findings can be used to improve insects' acceptability as an alternate protein source amongst consumers by improving the knowledge surrounding the defatting process during insect meal production and its effect on protein profile and functional properties.

\section{Pulsed electric field assisted extraction of valuable compounds from house crickets (Acheta domesticus)}

\author{
M. Psarianos ${ }^{1 *}$, G. Dimopoulos ${ }^{2}$, S. Bußler ${ }^{1}$, A.C. Cavini Moreno ${ }^{1,3}$, P. Taoukis $^{2}$, O. Shikha ${ }^{1}$ and O. Schlüter ${ }^{1}$ \\ ${ }^{1}$ Quality and Safety of Food and Feed, Leibniz Institute for Agricultural Engineering and Bioeconomy (ATB), Max-Eyth- \\ Allee 100, 14469, Potsdam, Germany; ${ }^{2}$ Laboratory of Food Chemistry and Technology, School of Chemical Engineering, \\ National Technical University of Athens (NTUA), Iroon Polytechniou 5, 15780 Athens, Greece; ${ }^{3}$ Faculty of Animal \\ Science and Food Engineering (FZEA), University of São Paulo (USP), Butanta, São Paulo, State of São Paulo, Brazil; \\ mpsarianos@atb-potsdam.de
}

Edible insects are traditionally consumed in several countries of the world due to their nutritional content. Extraction of their nutrients is an effective way to incorporate insects into food products. Application of pulsed electric field (PEF) processing has been suggested to optimise the extraction process of compounds from agricultural matrices. The present study investigates the application of PEF on crickets to optimise the extraction of their valuable components. Insect biomass was processed with PEF at several energy input conditions (4.9-49.10 kJ/kg) aiming to increase the protein and fat yield. Further, a synergistic effect of PEF and freeze drying was studied, with freeze dried and rehydrated material. Proteins were isolated using a solid-liquid extraction process (90 min, DI water, $\mathrm{pH}$ 10). Following centrifugation, the amount of soluble proteins in the supernatant was determined via Bradford method. Individual amino acid composition of the extracts was analysed using HPLC analysis. Fat was isolated via solid-liquid extraction ( $45 \mathrm{~min}$, n-hexane with teri-buolylated hydroxyl toluene). After centrifugation the fat was separated from the supernatant in a rotary evaporator. Individual fatty acid composition was determined with GC-MS following microwave assisted esterification process. Most intense PEF conditions (24.53-49.10 kJ/kg) led to highest yields of both components. Regarding the fresh material, fat and protein yields were increased by up to 53 and $100 \%$, respectively. Treating dried and rehydrated material did not increase the fat yield, while the protein yield was increased by $73 \%$. Further, the effect of PEF processing resulted in specific effects on amino acid and fatty acid composition. The present study explores the application of PEF treatment on insects and crickets in particular and indicates that PEF offers the potential of accelerating the extraction process of insect compounds. 


\title{
Bioaccumulation of cadmium in Tenebrio molitor, Zophobas morio and Hermetia illucens
}

\author{
C. Jucker ${ }^{1}$, F. Defilippo ${ }^{2}$, D. Lupi ${ }^{1}$, P. Gigante ${ }^{2}$, S. Savoldelli ${ }^{1}$, E. Ferretti ${ }^{2}$, M. Curatolo ${ }^{2}$, A. Bassi ${ }^{2}$, P. Bonilauri ${ }^{2}$ \\ and M. Dottori ${ }^{2}$ \\ ${ }^{1}$ Department of Food, Environmental and Nutritional Sciences, University of Milan, via Celoria 2, 20133 Milan, Italy; \\ 2IZSLER 'B. Ubertini,'Via Bianchi 9, 25124 Brescia, Italy; costanza.jucker@unimi.it
}

Insects have been proposed as a high quality, efficient and sustainable alternative protein source via feed or as a direct food source for humans. Mealworms and black soldier fly are already mass-produced as feed. One of the potential risks associated with their consumption is the chemical contamination (i.e. heavy metals). This study focuses on the potential bioaccumulation of cadmium from the feeding media in the mealworm species Yellow mealworm (Tenebrio molitor) and Giant mealworm (Zophobas morio) and in the Black soldier fly (Hermetia illucens). A colony of the three species was set up and maintained in a climate chamber. Larvae were used to define the chemical risk by assessing their storage capacity, when fed on artificially contaminated diet with cadmium at a concentration of $1.109 \mathrm{mg} / \mathrm{kg}$. The control substrate had an initial cadmium concentration of $0.030 \mathrm{mg} / \mathrm{kg}$ for $H$. illucens and 0.114 for the mealworms. For each trial $(\mathrm{n}=3)$, 300 larvae were used for $H$. illucens, while 50 for T. molitor and Z. morio. The bioaccumulation factor (BAF) was estimated for mature larvae in mealworm species. In BSF specimens the accumulation was tested at each larval instar, pupal and adult stage. Each sample was processed by inductively coupled plasma mass spectrometry. For BSF data showed a cadmium concentration, in larvae and in pupae, always greater than $2 \mathrm{mg} / \mathrm{kg}$, with a decrease in the last stages of development. The concentration of cadmium in the residual substrate tends to remain almost constant up to IV larval instar and then decrease during the prepupal and pupal stage. The BAF showed a substantial increase in the prepupal and pupal stage. Development times in contaminated diet were higher than the control group. In mealworm larvae, bioaccumulation was less relevant, with a value of 0.79 and 0.44 $\mathrm{mg} / \mathrm{kg}$ respectively. These values are below the values of the initial diet, both for the control larvae and for those fed on the artificially contaminated diet. No differences were noticed on the survival of the two mealworms between the contaminated and the control diet, while final larval weight of $Z$. morio was impacted from the presence of cadmium. The study reveals a high ability of $H$. illucens to accumulate cadmium, while in mealworms larvae, most likely the longer development allowed their elimination. As the accumulation of heavy metals varies according to the type of metal, the species of insects and the stage of development, for food safety it is important to evaluate the potential risks in a case by case approach.

\section{Safety aspects of using black soldier fly larvae as feed and food: the study of the bioaccumulation of metals}

\author{
T. Persaň", J. Kuta, J. Hofman and J. Vašíčková \\ Masaryk University, Faculty of Science, RECETOX, Kamenice 753/5, 62500 Brno, Czech Republic; tomas.persan@recetox.muni.cz
}

A world growing demand for protein sources originating from sustainable and environmentally friendly technologies (which are in line with ideas of EU/USA Green Deals) leads to exploration of new protein sources. Larvae of black soldier fly (BSF) (Hermetia illucens) seems the ideal candidate for the massive production due to fast growth rate, high content of protein and lipids, and valorisation of a wide variety of organic substrates. This species can be grown on different types of organic wastes such as brewers waste, manure, waste from restaurants, etc. The bioaccumulation of metals in insects is a very well-known phenomenon. However, before entering the market, it is crucial to explore the safety of insect products. This study explored the ability of bioaccumulation of metals and other elements in BSF depending on their diet. The study directly connects the rearing of BSF on different types of wastes and explores how these matrices affect the bioaccumulation of metals in BSF larvae. Moreover, the study discusses the possibilities to minimise contamination. The larvae were fed with eight different organic wastes such as peas, spent grain, oatmeal, rice, soybeans, breadcrumbs, curd, and fruit-vegetable mix. A comparison of the content of heavy metals in BSF reared on these eight different substrates was conducted. The content of 15 elements in fully-grown larvae was measured. Results were compared with legislative limits for undesirable substances in animal feed (Directive 2002/32/EC of the European Parliament) and risk assessment was performed. The developmental duration and bioconversion were monitored too. The results showed that the most accumulated elements were $\mathrm{Cd}$ and $\mathrm{Mn}$. For the future application of larvae as a feed, the accumulation of $\mathrm{Cd}$ in the insect is the most problematic phenomenon. Further research to ensure the safety of BSF products is necessary. This project was supported by the Technology Agency of the Czech Republic (TH04030169). 


\title{
Experimental study of drying characteristics of Cirina forda caterpillar
}

\author{
C. Bukamba Tshanga ${ }^{1,2^{*}}$, P. Malumba Kamba ${ }^{3}$, C. Van Engeland ${ }^{1}$, D. Mayele ${ }^{2}$, B. Kambashi ${ }^{4}$, J. Bindelle ${ }^{5}$ and \\ F. Debaste ${ }^{1}$ \\ ${ }^{1}$ Université Libre de Bruxelles (ULB), Transfers, Interfaces and Processes (TIPs), Avenue F.D. Roosevelt, 50 CP165/67, 1050 \\ Brussels, Belgium; ${ }^{2}$ Université de Kinshasa, Faculté des Sciences Agronomiques, Département de Chimie et Industries \\ Agricoles, Kenya; ${ }^{3}$ University of Liège, Gembloux Agro-Bio Tech, Care FoodIsLife, Avenue de la Faculté 2B, 5030, \\ Gembloux, Belgium; ${ }^{4}$ Université de Kinshasa, Faculté des Sciences Agronomiques, Département de Zootechnie, Kenya; \\ ${ }^{5}$ University of Liège, Gembloux Agro-Bio Tech, Terra AgricultureIsLife, Avenue de la Faculté 2, 5030, Gembloux, Belgium; \\ celestin.bukamba.tshanga@ulb.ac.be
}

The growth in world population is causing an increase of the global demand for food, and in particular of proteins sources. In this regard, edible insects are an attractive response to the increased quantity of food proteins needed in the future. Production of insects could increase food yields at low environmental cost reducing emissions of greenhouse gases and ammonia and contribute to environmental sustainability.. Insects can undergo processing for preservation or consumption such as drying, toasting, frying, roasting or boiling. In Africa, caterpillars like Cirina forda, are sun dried. The drying lasted 3 to 4 days in dry period and can go up to a week in rainy weather. In order to develop more efficient drying methods, it is necessary to master the drying characteristics of the caterpillars. The aim of this work was to investigate the drying characteristic and sorption-desorption isothermes of $C$. forda's caterpillar. To reach this goal alive caterpillar was purchased from various market at Kinshasa, Democratic Republic of Congo and dried. Before drying, caterpillars were characterised morphologically and stored in plastic bag at $-20^{\circ} \mathrm{C}$ until use. The drying was carried out in a tunnel dryer with real time mass measurement (every $10 \mathrm{~min}$.). The samples were dried at temperatures of 40,50 and $60^{\circ} \mathrm{C}$. Complementary, adsorption-desorption isotherms were done by DVS method for $25,30,40$ and $50^{\circ} \mathrm{C}$. Experimental data were fitted with BET model. The experimental kinetic results, expressed in moisture content and drying rates, were compared to a large range if mathematical model describing the kinetics. Ten semi-theoretical thin layer models were compared. The drying curves illustrate drying rate increases with temperature and absence of constant rate period. Newton model was the best model to predict the moisture evolution of C. forda during drying because it is simple and gives a good fit with correlation coefficient between 0.996-0.999 and RMSE between 0.03-0.19. No significant precision was provided by the other models. Sorption isotherms were type III and few hysteresis was observed. BET model give a good fitting with experimental data.

\section{Utilisation of ergot-infected feed by yellow mealworm (Tenebrio molitor) larvae}

\author{
A.D. Beaulieu", C. Ochoa-Sanabria and F. Buchanan \\ University of Saskatchewan, Saskatoon, SK S7H 5A8, Canada; denise.beaulieu@usask.ca
}

Ergot alkaloids (EA) are secondary metabolites produced by the fungus Claviceps purpurea. Consumption of ergot-contaminated feed by livestock reduces feed intake and growth and may cause gangrene, agalactia, gastrointestinal ulcers and death. The Canadian Food and Inspection Agency currently recommends that diets contain a maximum of $4 \mathrm{mg} / \mathrm{kg}$ ergot alkaloids for swine, $6 \mathrm{mg} / \mathrm{kg}$ for poultry and $2 \mathrm{mg} / \mathrm{kg}$ for ruminants. Recently, we showed that yellow mealworm larvae (YML; Tenebrio molitor) could be grown on wheat contaminated with $12 \mathrm{mg} / \mathrm{kg}$ of the mycotoxin, deoxynivalenol (DON) while accumulating only low levels of DON. The objective of the current experiment was to evaluate the possibility of using EA-contaminated wheat as a feed source for YML. Larvae were placed in humidified plastic bins and provided diets containing contaminated wheat screenings to achieve a final content of $<1,4,8$ and $15 \mathrm{mg} \mathrm{EA} / \mathrm{kg}$ for the control, low, medium and high treatments, respectively. There were 150 YML per bin and 5 replicates per diet. Survival, weight of worms and feed was determined weekly for $21 \mathrm{~d}$. Larvae were then transferred to plastic glasses and fasted for $48 \mathrm{~h}$ to allow collection of frass. The content of 6 EA in the larvae, diets and frass were determined at Prairie Diagnostic Services, Saskatoon, SK by HPLC-MS. Preference of the larvae for EA was determined by placing 30 naïve worms into the centre of petri dishes with $2 \mathrm{~g}$ of each diet in one of 4 quadrants. Dishes were incubated for $20 \mathrm{~min}$ in the dark. The number of worms which had migrated to each quadrant was then counted. This process was repeated 5 times with each replicate. Data were analysed as a CRD using Mixed models with diet and bin as fixed and random effects, respectively. Diet means were separated using Tukey-Kramer HSD. Significance was declared at $P<0.05$. The pooled replicates of frass were not analysed statistically. Survival (\%) at d $7(94.1 \pm 1.17$; mean \pm SEM), d 21 (88.5 \pm 1.56$)$, average daily gain $(1.3 \pm 0.16 \mathrm{mg} / \mathrm{d} /$ larvae), or feed conversion ratio (14.4 $\pm 1.9 ; \mathrm{ADG} / \mathrm{feed} \times 100)$ were unaffected by diet $(P>0.10)$. Feed intake ( $\mathrm{g} / \mathrm{d} / \mathrm{bin})$ was higher in worms on the control diet compared to the EA contaminated diets (30.1 vs $23.8 \pm 1.5 ; P<0.05)$. The concentration of EA had no effect on diet preference $(P>0.50)$. Very low concentrations of EA were detected in the larvae $(0.15 \mathrm{mg} / \mathrm{kg}$; high diet). The frass contained $0.02,0.25,0.72$ and $1.3 \mathrm{mg} / \mathrm{kg}$ EA for the control, low medium and high diets, respectively. Ergometrine and ergocryptine were the major EA determined in the diet and faeces, respectively. In conclusion, YML can be grown on, and apparently decontaminate, wheat-based diets contaminated with up to $15 \mathrm{mg} / \mathrm{kg}$ EA. 


\title{
Insect as feed/alternative protein sources \& diet formulations
}

\section{Live insect larvae for laying hens: effects on egg quality, feather conditions and animal behaviour}

\author{
S. Bellezza Oddon ${ }^{1 *}$, I. Biasato ${ }^{1}$, A. Imarisio ${ }^{2}$, M. Pipan $^{3}$, D. Dekleva ${ }^{3}$, S. Dabbou ${ }^{4}$, M. Renna ${ }^{2}$, G. Chemello ${ }^{1}$, \\ M. Gariglio ${ }^{2}$, C. Caimi ${ }^{1}$, E. Fiorilla ${ }^{1}$, L. Gasco ${ }^{1}$ and A. Schiavone ${ }^{2}$ \\ ${ }^{1}$ Dept. of Agricultural, Forest and Food Sciences, University of Turin, Italy; ${ }^{2}$ Dept. of Veterinary Science, University of Turin, \\ Italy; ${ }^{3}$ Entomics Biosystems, Cambridge, United Kingdom; ${ }^{4}$ Center Agriculture Food Environment, University of Trento, \\ Italy; sara.bellezzaoddon@unito.it
}

Feather pecking is widespread in intensive laying hens farming, negatively impacting performance, welfare and product quality. By increasing foraging behaviour, live insect larvae can divert birds' attention, reducing damage from injurious pecking and having positive repercussions on welfare. This study aimed to evaluate the effects of live black soldier fly (Hermetia illucens) larvae on two slow-growing Italian breeds Bionda Piemontese (BP) and Bianca di Saluzzo (BS). Laying hens behaviour, feather conditions, and product quality were evaluated. 169 laying hens and 24 males of BP and BS breeds (308 days of age; sex ratio 1:8) were allotted to 12 pens and fed one of two dietary treatments (3 replicates/treatment, from 12 to 19 birds/replicate) as follows: (1) control diet (C): commercial feed; and (2) HI: C + H. illucens live larvae. Live larvae were distributed as a supplement based on $6 \%$ of the expected daily feed intake. Feathering scores were given to all birds. Hen behaviour was video recorded and divided into 2 categories: duration (walking, ground pecking, standing still, resting) and frequencies (scratching, grooming, allogrooming, trotting, severe feather pecking, stretching, chasing, sand bath, feather shake, wing flapping). Eggs were collected in 3 different periods and were analysed to determine: egg weigh, length, and width; shell weight; albumen weight; yolk weight and colour. Data were analysed by General Linear Mixed Model (IBM SPSS Statistics V20.0.0, P<0.05). No significant differences were observed for both the mean and the total feathering scores between the $\mathrm{C}$ and the $\mathrm{HI}$ hens independently of the breed. On the contrary, the HI BP animals showed improved feathering scores when compared to the $\mathrm{C}$ group $(P<0.05)$. The HI birds spent more time walking than the $\mathrm{C}$ group $(P<0.05)$. Considering the frequencies, the HI hens displayed more grooming, allogrooming and trotting than the $\mathrm{C}$ birds. On the contrary, the $\mathrm{C}$ group performed severe feather pecking than the HI hens. Egg, shell, albumen and yolk weights, as well as egg length and width more, and yolk colour, were similar in HI and control group. The use of live larvae as environmental enrichment positively affects the plumage status in the BP breed. Furthermore, the addition of live larvae to the commercial diet did not negatively affect eggs quality but leads to a decrease in severe feather pecking and an increase in some comfort behaviours. 


\title{
Broilers, black soldier fly larvae and behaviour
}

D.G.A.B. Oonincx ${ }^{1,2^{*}}$, C. Vreugdenhil ${ }^{3}$, T. Veldkamp ${ }^{4}$, V. Fogliano ${ }^{5}$, A. Dijkslag ${ }^{6}$, J.J.A. van Loon ${ }^{2}$ and T.B. Rodenburg $^{3,7}$ ${ }^{1}$ Animal Nutrition Group, Dept. of Animal Sciences, Wageningen University and Research, P.O. Box 383, 6700 AJ Wageningen, the Netherlands; ${ }^{2}$ Laboratory of Entomology, Dept. of Plant Sciences, Wageningen University and Research, P.O. Box 16, 6700 AA Wageningen, the Netherlands; ${ }^{3}$ Behavioural Ecology Group, Dept. of Animal Sciences, Wageningen University and Research, Wageningen, the Netherlands, ${ }^{4}$ Wageningen Livestock Research, Wageningen University and research, P.O. Box 338, 6700 AH Wageningen, the Netherlands; ${ }^{5}$ Food Quality and Design Group, Wageningen University and Research, 6700 AA Wageningen, the Netherlands; ${ }^{6}$ ForFarmers N.V., P.O. Box 91, 7240 AB Lochem, the Netherlands; ${ }^{7}$ Animals in Science and Society, Faculty of Veterinary Medicine, Utrecht University, Utrecht, the Netherlands; dennis.oonincx@wur.nl

Various studies have investigated the use of black soldier fly (BSF) larvae as a protein-rich ingredient in livestock diets. These studies mostly focused on effects on product quality and on production parameters such as feed conversion efficiency, growth and digestibility. More recently, aspects such as gut health and morphological effects have gained interest. The current study included product quality parameters and quantification of growth and feed conversion efficiency, but also assessed behaviour and welfare of broilers provided with live BSF larvae. The experimental setup consisted of one control and three treatment groups in which 5, 10 or 15\% of the DM was provided as live BSF larvae. Pens with groups of 7 male and 7 female one day old broiler chicks were used as experimental units and each treatment was replicated 17 times. In the larval treatments consumption started within 1 second after provision from the second week onwards. Furthermore, the number of pecks per minute increased linearly over time, indicating that the BSF larvae were well accepted. Inclusion of $5 \%$ live BSF larvae decreased feed conversion ratio compared to controls. However, inclusion of BSF larvae decreased total feed intake and consequently resulted in lower final weights in a dose dependent way. Whereas in all treatments activity levels decreased over time, this was more apparent in the control group than in the BSF supplemented treatments. Broilers provided with BSF larvae stood and walked more, and rested less. They also spent less time drinking water independent of the larval inclusion percentage. Larvae fed broilers also had lower incidences of foot pad dermatitis and hock burns. The provision of BSF larvae affected meat colour and pH. Lastly, water holding capacity of the chicken breast was elevated in the $10 \%$ inclusion group. As differences in growth were apparent between treatments, the aforementioned effects might partially be due to weight differences rather than BSF provision per se.

\section{Growth performance, carcass traits and profitability of black soldier fly larvae and Desmodium diets in broiler chicken}

\author{
M.M. Mutisya ${ }^{1,2 "}$, J.N. Kinyuru ${ }^{1}$, K.M. Agbodzavu ${ }^{2}$, C. Tanga ${ }^{2}$, M. Gicheha ${ }^{1}$, S. Ekesi ${ }^{2}$ and S. Niassy ${ }^{2}$ \\ ${ }^{1}$ Jomo Kenyatta University of Agriculture and Technology (JKUAT), Zoology Department, P.O. Box 62000-00200 Nairobi, \\ Kenya; International Centre of Insect Physiology and Ecology (icipe), Plant Health Unit, P.O. Box 30772-00100, Nairobi, \\ Kenya; mariahmutisya@gmail.com
}

The poultry industry is one of the fastest growing sectors more specifically in the African continent. Unavailability of quality, affordable, and sustainable protein rich sources is a major challenge and other than fishmeal and soybean, there is need to explore other alternatives such as insects and legume-based protein sources. In this study, we explored the effect of the black soldier fly larvae (BSFL) and Desmodium based diets on the growth performance, carcass traits and their profitability in broiler chicken. Desmodium and BSFL powders were formulated in three rations as follows: T1 25:75; T2 50:50 and T3 75:25. A commercial feed was used as a control. One hundred and twenty mixed sex day old broiler chicks (120-cobb) were reared in cages under the same conditions for 42 days in a completely randomised design. Growth performance data was collected weekly by weighing the birds and taking measurements of various body parts. The birds were thereafter slaughtered for carcass quality evaluation and recording of the weights of internal organs. Chicken fed with T2 (50:50) formulation had significantly higher growth rate in terms of weight gain. At slaughter, T1 and control chicken had the highest live weights though no significant differences between treatments. All Desmodium-BSFL fed chicken had higher thigh weights compared to the control, though no significant differences were found. Likewise, all treatments had higher breast weights compared to the control. T3 (25:75) presented the highest neck weight with significant differences from the other treatments. Liver and gizzard weights were generally higher in all Desmodium-BSFL fed chicken compared to the conventional feeds (control diet) fed chicken. We found that Desmodium-BSFL based feeds are more profitable than the control feed and the assorted mode of sale generates more revenue compared to when the chicken are sold on whole chicken basis at either doorstep or retail. The study suggests that Desmodium-BSFL mixtures are promising feed alternatives for smallholder farmers and should be promoted for a more vibrant poultry sector. 


\title{
Full fat black soldier fly larvae meal diets enhances egg production and profitability of smallholder farmers in Kenya
}

\author{
C.M. Tanga ${ }^{1 *}$, D.W. Nyingi ${ }^{1,2}$, I.M. Osuga ${ }^{1,6}$, L.K. Munga ${ }^{2}$, S. Subramanian ${ }^{1}$, J.P. Egonyu ${ }^{1}$, D.M. Mwangi ${ }^{3}$, \\ D. Nakimbugwe ${ }^{4}$, J.J.A. van Loon ${ }^{5}$, M. Dicke ${ }^{5}$ and S. Ekesi ${ }^{1}$ \\ ${ }^{1}$ International Centre of Insect Physiology and Ecology, P.O. Box 30772-00100 Nairobi, Kenya; ${ }^{2}$ Department of Animal Science, \\ Kenyatta University, P.O. Box 34844-00100, Nairobi, Kenya; ${ }^{3}$ Kenya Agricultural and Livestock Research Organization, P.O. \\ Box 25-20117, Naivasha, Kenya; ${ }^{4}$ Department of Food Technology and Nutrition, School of Food Technology, Nutrition E \\ Bioengineering, Makerere University, P.O. Box 7062, Kampala, Uganda; ${ }^{5}$ Laboratory of Entomology, Wageningen University, \\ P.O. Box 16, 6700 AA Wageningen, the Netherlands; ${ }^{6}$ Department of Animal Science, Jomo Kenyatta University of Agriculture \\ and Technology, P.O. Box 62000-00200, Nairobi, Kenya; ctanga@icipe.org
}

In recent years, the development of indigenous chicken production has been a sustainable way of helping to meet the livelihood needs of rural populations and raise their living standards. However, regular supply of high-quality feeds is crucial to improve growth and laying performance of these birds. The prohibitive costs of fishmeal-based diet for poultry has hampered the growth of the sector. This study sought to evaluate the effects of substituting the expensive fishmeal (FM) with black soldier fly larvae meal (BSFLM) on the weight change, egg production and egg characteristics of improved indigenous chicken (KALRO-Kienyeji). Fifty-five (55) hens aged 20 weeks-old were randomly assigned to five iso-energetic and iso-nitrogenous diets with different inclusion levels (0, 25, 50, 75 and 100\%) of BSFLM to substitute FM. Each treatment was replicated 11 times. The average daily feed intake, average daily weight change, feed conversion ratio and overall weight gain varied significantly when birds were subjected to the various diets. Egg production was significantly affected by the various dietary treatments. Replacement of FM with 100\% BSFLM had the highest and prolonged laying percentage (84.2\%) compared to the control (100\% FM) diet (54.0\%). The weight of the eggs collected ranged between 57-63 g, with significantly higher values recorded for hens fed 75\% BSFLM inclusion compared to the control diet. Birds fed on diets with 100\% BSFLM inclusion rates had the highest egg yolk colour intensity index, while the control had the lowest index. The gross profit margin, cost-benefit ratio and return of investment were significantly higher for hens provided diets with BSFLM inclusion. Our findings highlight the contribution of insect-based feeds in sustainable poultry production and provide opportunities for smallholder farmers in lowand middle-income countries to increase productivity, improve their livelihood and contribute to food and nutrition security.

\section{Post-processing and marketing of black soldier fly conversion products - the case of East Java}

\author{
J. Egger", E. Antarest, B.M.A. Dortmans and C. Zurbrügg \\ Eawag - Swiss Federal Institute of Aquatic Science and Technology, Department of Sanitation, Water and Solid Waste for \\ Development (Sandec), 8600 Dübendorf, Switzerland; julia.egger@eawag.ch
}

Waste treatment with black soldier fly larvae (BSFL) converts biowaste into valuable insect biomass. The technology is popular given the possible revenues from sales of BSFL derived products such as BSFL based animal feed. Revenues from product sales can provide the incentive to use this approach for biowaste management, especially in low-and middle-income settings with lacking waste infrastructure and services. We present results of the SIBRE project in East-Java, Indonesia that focuses on developing various animal feed products from BSFL and assesses their market potential for the case of a small-scale BSF facility located in Sidoarjo. Given the scale of operation, the market assessment identified the local pet food market (bird, ornamental fish and reptile) as a promising market for sales of BSFL based animal feed products. The market value for such animal feed is estimated at around one million US dollars per month (for the city of Surabaya) with around 40 products that could be substituted by BSFL. Based on these findings different post-processing pathways for BSFL were researched for a variety of BSFL products. Results are presented in a post-processing matrix. Acceptance and competitiveness of these newly developed products were tested by supplying them in the local market to retailers at sales prices based on production cost at the currently operating BSF waste treatment facility. Independent of the product envisaged, post-processing starts with a blanching step to reduce microbial load which also prevents colour changes during subsequent drying. The next processing step involves drying either using a microwave to produce what we call 'pop-larvae' or in a dehydrator $\left(65^{\circ} \mathrm{C}, 20 \mathrm{~h}\right)$. Microwaved larvae (1000 W, $15 \mathrm{~min}$ ) seem to be more appealing to customers and animals given the crunchy, light texture and puffed shape. These sensory benefits allow a higher selling price and thus this compensates for the higher production costs. Mechanical oil extraction from dried larvae allows yield of a defatted protein meal as well as BSFL oil. Given the production costs of BSFL meal and oil, selling prices are higher than the price of fish meal and fish oil available in East Java. The high protein meal could be mixed in formulations for higher priced exotic fish feed pellets. The fatty acid profile of BSFL oil is similar to palm kernel oil or coconut oil, which are commonly used in cosmetic industry. Natural based cosmetics are a trend in East Java with higher selling prices, which could therefore warrant the use of BSFL oil. 


\title{
Assessing the impact of live black soldier fly larvae supplementation in laying hen diets
}

\author{
D. Dekleva ${ }^{1}$, A.R.L. Charbonneau ${ }^{1}$, P.V. Crespo ${ }^{1}$, N. Wan ${ }^{1}$, M. Monticelli ${ }^{1}$, N. Mackie ${ }^{2}$, J. Tarlton ${ }^{2}$ and M. Pipan ${ }^{1 *}$ \\ ${ }^{1}$ Entomics Biosystems Limited, Cambridge, United Kingdom; ${ }^{2}$ Bristol Veterinary School, University of Bristol, Langford, \\ United Kingdom; miha.pipan@entomics.com
}

Black soldier fly (Hermetia illucens, BSF) represents a species of particular commercial interest given its ability to convert a broad range of biomass into quality nutrients and materials. Utilisation of insect meals in monogastric livestock diets, including BSF larvae meal, has been the subject of many research publications over the past few years and shows great promise in terms of delivering quality nutrition. This study explored the impacts of supplementing commercial laying hen diets with live BSF larvae. Commercial point-of-lay brown layers were fed ad libitum with a commercial layer crumb, and split into two groups: control hens (CON) who were fed solely on the commercial diet, and insect-fed hens (IF) whose diets were supplemented with $5 \% \mathrm{w} / \mathrm{w}$ live BSF larvae ration delivered daily in addition to the core feed. Eight rooms of 100 hens each were trialled this way over 15 weeks, while data from: (1) 20 focus hens from each room were inspected weekly, their weights, feather scores, health monitored and logged; (2) laid eggs (nest/floor) were counted, weighted and classed, samples were also taken for nutritional, yolk/white quality, shell strength and shelf life analyses; (3) behaviour and vocalisation were recorded. At the end of the trial, 5 hens per room were sacrificed and their tissues sampled for further analyses (blood, gut and liver histology, microbiome, gene expression). Evidence collected suggests live insect supplementation practices using BSF larvae are an effective way to improve laying hen welfare \& quality of life, showing a beneficial improvement in many of the analysed health markers. Egg size, quality and shelf life were not affected - the sole observed change was an increase in medium chain fatty acids in egg yolks from IF hens. The trial setup resulted in IF laying hens producing more eggs than CON hens, suggesting productivity increases may be achieved with this manner of commercial layer diet supplementation.

\section{Black soldier fly meal inclusion in rainbow trout diets: effects on growth performances from juvenile to portion-size}

\author{
C. Guidou ${ }^{1 *}$, C. Trespeuch ${ }^{1}$, J. Dias ${ }^{2}$, L. Lesur ${ }^{3}$, C. Masset ${ }^{3}$ and Y. Marchand ${ }^{3}$
}

${ }^{1}$ Mutatec, 4 rue Toussaint Flechaire, 84510 Caumont-sur-Durance, France; ${ }^{2}$ SPAROS LDA, Área Empresarial de Marim, 8700-221 Olhão, Portugal; ${ }^{3}$ Le Gouessant, Z.I., 1 rue de La Jeannaie, 22402 Lamballe, France; c.guidou@mutatec.com

For several years, feed manufacturers are searching for new sources of proteins in order to respond to the growth of the sector and the challenges of sustainable development. At the same time, more than $30 \%$ of the world agricultural production is wasted (unsold or expired products, by-products of agri-food industries). Recommended by the Food and Agriculture Organization of the United Nations (FAO), insects make it possible to valorise food waste (bioconversion). The black soldier fly (Hermetia illucens) represents a particularly adapted resource to feed fish because it is natural, safe, sustainably produced and has a good nutritional quality. The objective of the studies carried out between December 2018 and May 2019 is to evaluate the zootechnical performances of trout when a part of fishmeal is replaced by a partially defatted black soldier fly (BSF) meal at different inclusion levels, at diverse trout sizes and under various breeding conditions. A BSF meal, produced by a French company, is incorporated in feeds of different sizes $(1.2,2,5 \mathrm{~mm}$ ) and at different inclusion rates (5 to $25 \%$ ), as replacement material for fishmeal. These feeds have been used to feed rainbow trout (Oncorhynchus mykiss) from 7 grams to 350 grams in four locations in France and in the European Union, in experimental facilities and in fish farms. All results were positive. Trout fed with feeds containing BSF meal showed faster growth and better feed conversion ratios than the control groups fish. It therefore seems possible to replace a significant portion (up to 60\%) of fishmeal by BSF meal. During these studies, complementary analyses have been undertaken: nutritional composition of the fish, digestibility of the feed and effects on the fish health. 


\title{
Apparent digestibility coefficient of cockroach (Periplanata americana) meal by Clarias gariepinus
}

\author{
S.O. Sule ${ }^{1,2^{*}}$, A.O. Sotolu ${ }^{3}$ and O.S. Yakubu ${ }^{4,5}$ \\ ${ }^{1}$ Department of Forestry, Wildlife and Fisheries, Faculty of Agricultural Production and Renewable Resources, College of \\ Agricultural Sciences, Olabisi Onabanjo University, Ayetoro Campus, Ogun State, Nigeria; ${ }^{2}$ Department of Aquaculture and \\ Fisheries, University of Ibadan, Ibadan, Oyo State, Nigeria; ${ }^{3}$ Department of Aquaculture and Fisheries Management, Faculty \\ of Agriculture, Shabu-Lafia Campus, Nasarawa State University, Keffi, Nasarawa State, Nigeria; ${ }^{4}$ Institute of Aquaculture, \\ University of Stirling, Stirling FK9 4LA, Scotland, United Kingdom; ${ }^{5}$ Department of Fisheries, Federal University Gashua, \\ Yobe State, Nigeria; sule.okanlawon@oouagoiwoye.edu.ng
}

Insect consumption has been relished by man and animal. The high cost of fishmeal in aquaculture diet has led to the search of renewable resources as animal protein source in fish nutrition. Cockroaches are ubiquitous insect and its farming is on the increase as source of dietary protein and pharmaceuticals. Cockroach (Periplanata americana) meal digestibility was studied using Clarias gariepinus. Diet of $40 \%$ protein basal and test diet with inert biomarker was used to assess the digestibility of the meal at 70:30 ratios over 42 day period using ten fish/tank/replicate of average weight $29.22 \mathrm{~g}$. Digestibility coefficient in test fish and basal fish revealed the following: protein (88.48 vs $87.01 \%)$; dry matter (87.35 vs $86.82 \%)$, energy (86.88 vs $85.48 \%$ ); fat (39.67 vs $27.75 \%$ ). Growth response to diets revealed test and basal fed fish; weight gain (52.59 vs $36.31 \mathrm{~g}$ ); Sgr ( 0.03 vs $0.02 \mathrm{~g}$ ); feed conversion ratio (1.71 vs 2.13 ) and percentage weight gain (181.44 vs $123.35 \%)$ respectively. The values of test diet were significantly higher $(P<0.05)$ than basal diet indicating the suitability of the meal in dietary inclusion for fish.

\section{Different production pathways of insect meal and the effects in feed formulations}

\author{
V. Böschen \\ Research Institute of Feed Technology (IFF), Frickenmühle 1A, 38110 Brunswick, Germany; v.boeschen@iff-braunschweig.de
}

In order to use insect meal as animal feed, it must be processed and defatted in different ways. Various thermal / mechanical processes are used for this. From the field of soy pretreatment (toasting) it is known that excessive pretreatment can have negative effects on animals, or that it can have economic consequences for feed manufacturers in the form of amino acid addition. This was confirmed in growth experiments of an AiF project. The effects of the substitution of soy protein by insect protein (Tenebrio molitor) on acceptance, feed conversion, growth performance and feed protein quality in broilers were investigated. A first indication was a feeding experiment in which soy extraction meal was replaced by T. molitor meal in the feed ration and there was no amino acid addition. Both in the starter and in the grower phase when looking at the entire test period with regard to the growth parameters no significant differences were found between the feed mixtures. The feed mixture with T. molitor meals, however, showed significantly lower values for all parameters. The final live weight of the animals without amino acid supplement was only half that as with feed mixture with amino acid supplement. Since the composition of the protein carrier (T. molitor meal) can change due to the different processing routes up to the protein flour, the amino acid composition of the protein carrier and the content of soluble protein were investigated using different processing strategies (drying temperatures). For the experiments a belt drying system was used to dry the T. molitor larvae. The only variation parameter during drying was the temperature; other parameters such as the belt load or the air speed were kept constant. Similar to the overheating of soybeans or soy feed, the essential amino acids such as lysine, cysteine and valine are reduced as the temperature rises. 


\title{
The effect of soybean oil replacement by Hermetia illucens fat on young turkey performance and nutrient digestibility
}

\author{
J. Sypniewski ${ }^{1}$, Z. Mikołajczak ${ }^{2}$, B. Kierończyk ${ }^{2 *}$, M. Rawski ${ }^{3}$, W. Czekała ${ }^{4}$ and D. Józefiak $^{2}$ \\ ${ }^{1}$ PIAST GROUP Research and Development Center, Lewkowiec 50A, 63-400 Ostrów Wielkopolski, Poland; ${ }^{2}$ Department \\ of Animal Nutrition, Poznań University of Life Sciences, Wotyńska 33, 60-637 Poznań, Poland; ${ }^{3}$ Division of Inland \\ Fisheries and Aquaculture, Institute of Zoology, University of Life Sciences, Wojska Polskiego 71C, 60-637 Poznań, \\ Poland; Institite of Biosystems Engineering, University of Life Sciences, Wojska Polskiego 50, 60-637 Poznań, Poland; \\ bartosz.kieronczyk@up.poznan.pl
}

The aim of the present study was to investigate the effect of partial or total replacement of soybean oil by black soldier fly larvae (BSFL) fat obtained using super-critical extraction with $\mathrm{CO}_{2}$ on the growth performance and coefficients of apparent nutrient digestibility of young turkey poults. A total of 216 seven-day-old female turkeys (B.U.T. 6) were randomly allocated to 3 dietary treatments contained 6 replications (12 birds each). The birds were kept in floor pens from day 7 till $35 \mathrm{~d}$ of age. The following treatments were applied: SO - 100\% of soybean oil, HI50 - 50\% of BSFL fat and 50\% of SO, and HI100 - 100\% of BSFL fat. The body weight gain (BWG) and feed intake (FI) were measured, and the feed conversion ratio (FCR) was calculated on days 21 and 35. Coefficients of apparent ileal digestibility of crude protein (CP), ether extract (EE), and apparent metabolisable energy corrected to zero nitrogen balance (AMEN) in turkeys were calculated relative to the ratio of $\mathrm{TiO}_{2}$. The selected internal organ weight in relation to body weight (BW) (\% of BW), and lengths in relation to $\mathrm{BW}(\mathrm{cm} / \mathrm{kg} \mathrm{BW}$ ), were measured. The digesta samples from the duodenum were collected for pancreatic enzyme examination, i.e. lipase and trypsin (d35). The obtained results of soybean oil replacement by BSFL fat confirmed previous experiments carried out on broiler chickens. The partial or total replacement by BSFL oil have shown similar growth performance results to the $\mathrm{SO}$ treatment in the 7-35 d period. It stays in agreement with the coefficients of nutrients digestibility which were not affected $(P>0.05)$ by the BSFL. However, trypsin activity was decreased $(P=0.002)$ by BSFL100. Moreover, the addition of BSFL fat to the turkey's diet did not affect $(P>0.05)$ selected internal organ weights, as well as the gastrointestinal tract length. In conclusion, the BSFL fat may be an alternative source of energy used in young turkey nutrition. This study was performed in the frame of the IN-OIL project: An innovative method for bio-conversion of by-products from food processing industry that was financed by the National Centre for Research and Development within the LIDER VII Programme No. 0148/L-7/2015.

\section{Partially defatted black soldier fly meal inclusion in juvenile rainbow trout diets: effects on growth performances}

\section{Guidou ${ }^{1 *}$, C. Trespeuch ${ }^{1}$ and J. Dias ${ }^{2}$}

${ }^{1}$ Mutatec, 4 rue Toussaint Flechaire, 84510 Caumont-sur-Durance, France; ${ }^{2}$ SPAROS LDA, Área Empresarial de Marim, 8700-221 Olhão, Portugal; c.guidou@mutatec.com

For several years, feed manufacturers are searching for new sources of proteins in order to respond to the growth of the sector and the challenges of sustainable development. At the same time, more than $30 \%$ of the world agricultural production is wasted (unsold or expired products, by-products of agri-food industries). Recommended by the Food and Agriculture Organization of the United Nations (FAO), insects make it possible to valorise food waste (bioconversion). The black soldier fly (Hermetia illucens) represents a particularly adapted resource to feed fish because it is natural, safe, sustainably produced and has a good nutritional quality. The objective of the study carried out between December 2018 and May 2019 is to evaluate the zootechnical performance of juveniles trout when a part of fishmeal is replaced by a partially defatted black soldier fly (BSF) meal at different inclusion levels in comparison to a conventional feed (CTRL) and a feed containing another insect, the mealworm (TM). A BSF meal, produced by a French company, is incorporated in feeds of different sizes $(1.2 \mathrm{~mm} ; 2 \mathrm{~mm})$ and at different inclusion rates ( 5 to $25 \%$ ), as replacement material for fishmeal. These feeds have been used to feed rainbow trout (Oncorhynchus mykiss) from 7 grams to 65 grams ( 86 days of trial). All results were positive. Trout fed with feeds containing BSF meal showed faster growth and better feed conversion ratios than the CTRL group fish. It therefore seems possible to replace a significant portion (up to 60\%) of fishmeal by BSF meal. At equivalent fishmeal replacement rate, results with BSF meal are superior ( $+7 \%$ mean body weight) than results with TM meal (non-significant). Other trials in experimental stations and in fish farms showed that the replacement of a part of the fishmeal by a BSF meal improves the growth performance of the rainbow trout during the growing period (120-350 g). 


\title{
Evaluation of Zophobas morio larvae meal as fishmeal replacer for gilthead seabream diet
}

\author{
A. Asimaki ${ }^{1}$, P. Psofakis ${ }^{1}$, G. Ekonomou ${ }^{1}$, E. Mente ${ }^{1}$, C.I. Rumbos ${ }^{2}$, C.G. Athanassiou ${ }^{2}$, E. Fountoulaki ${ }^{3}$, M. Henry ${ }^{3}$ \\ and I.T. Karapanagiotidis ${ }^{*}$ \\ ${ }^{1}$ Aquaculture Laboratory, Dept. of Ichthyology and Aquatic Environment, University of Thessaly, Volos, Greece; ${ }^{2}$ Laboratory \\ of Entomology and Agricultural Zoology, Dept. of Agriculture, Crop Production and Rural Environment, University of \\ Thessaly, Volos, Greece; ${ }^{3}$ Hellenic Centre for Marine Research, Athens, Greece; ikarapan@uth.gr
}

The dietary inclusion levels of fishmeal as a major protein source in aquafeeds are decreasing due to its stagnant availability in the global market, its rising price and the environmental concerns that are linked to its production. Moreover, as aquaculture further develops and intensifies, the need for alternative protein sources in aquafeeds becomes more intense. The use of insect meals for the replacement of fishmeal in aquafeeds has attracted the research interest, especially after their recent approval in the European aquafeed chain. Among the insect species used so far, most studies have been conducted with Tenebrio molitor and Hermetia illucens. However, other species that have not yet been studied extensively could also be proved suitable for fishmeal replacements. The giant mealworm, Zophobas morio, is a large tenebrionid beetle species, with high nutritive value, which is commonly reared as feed for birds and reptiles. In the present study, we examined the suitability of $Z$. morio as fishmeal replacer in gilthead seabream (Sparus aurata) diet. Seabream is amongst the most important marine fish species reared in Europe with an annual world production of around 160,000 mt. Z. morio larvae were raised in a mixture of wheat bran (90\%) and egg layer hens feed (10\%). Late-instar larvae were collected, dried and milled, in order to produce the Z. morio larvae meal (12\% moisture, $41 \%$ crude protein, $39 \%$ crude lipid) used in the feeding trial. A total number of 270 S. aurata juveniles of $3.4 \mathrm{~g}$ initial mean weight were allocated into 9 glass tanks (125l) within a closed recirculation seawater system and divided into 3 dietary groups in triplicates, each feeding on a different diet. Three isonitrogenous (52\%) and isoenergetic (20 MJ/ $\mathrm{kg}$ ) diets were formulated at which the fishmeal protein of the control diet was replaced by the Z. morio meal at 5 and $10 \%$ ). Fish were fed to satiation twice a day, 6 days per week for 100 days in total. High survival rates, reaching $99 \%$, were recorded in all groups. In addition, no significant differences were noted among all fish groups for feed intake, final weights, specific growth rates and FCR. These findings suggest that $Z$. morio is an attractive feedstuff that could successfully replace fishmeal protein in seabream's diet at 10\%. The study was funded by 'Operational Programme Competitiveness, Entrepreneurship and Innovation 2014-2020 (EPAnEK)' of Hellenic Republic.

\section{Effect of soybean oil replacement by Hermetia illucens fat on broiler chicken performance and nutrients digestibility}

\author{
Z. Mikołajczak ${ }^{1}$, B. Kierończyk ${ }^{1 *}$, M. Rawski ${ }^{2}$, W. Czekała and D. Józefiak ${ }^{1}$ \\ ${ }^{1}$ Department of Animal Nutrition, Poznań University of Life Sciences, Wotyńska 33, 60-637 Poznań, Poland; ${ }^{2}$ Division of \\ Inland Fisheries and Aquaculture, Institute of Zoology, University of Life Sciences, Wojska Polskiego 71C, 60-637 Poznań, \\ Poland; ${ }^{3}$ Institite of Biosystems Engineering, University of Life Sciences, Wojska Polskiego 50, 60-637 Poznań, Poland; \\ bartosz.kieronczyk@up.poznan.pl
}

The aim of the study was to investigate the effect of partial or full replacement of soybean oil by Hermetia illucens fat (HI) obtained via super-critical $\mathrm{CO}_{2}$ extraction on the growth performance and nutrients digestibility of broiler chickens. In an experiment, lasted $35 \mathrm{~d}$ 1-day-old male broilers (Ross 308) were used. The birds were assigned to 5 groups per 10 replicates (16 birds per rep). Food and water were supplied ad libitum. The following design of the experiment was used: SO; control treatment with $100 \%$ soybean oil; HI25, 50, 75, 100; experimental treatments with partial, i.e. 25, 50, 75 or full 100 replacement of soybean oil by $\mathrm{HI}$ fat. $\mathrm{TiO}_{2}(0.3 \%)$ as an internal marker was used. The growth performance parameters, i.e. body weight gain (BWG), feed intake (FI) were measured and feed conversion ratio (FCR) was calculated at 14, 21, 28, and $35 \mathrm{~d}$, as well as coefficients of apparent ileal digestibility of nutrients, were calculated at $35 \mathrm{~d}$ of age. In general, there were no statistically significant differences $(P>0.05)$ between control SO and experimental groups in terms of BWG, FI, as well as FCR. Only in the first two weeks (1-14 d) HI oil positively decreased FI $(P<0.001)$, and FCR $(P=0.031)$ values in comparison to SO control group. However, in the case of FCR the most efficient were groups with 75 or $100 \%$ of $H$. illucens fat in the broiler diets. The results of digestibility calculation at $35 \mathrm{~d}$ were not affected by $H$. illucens fat, in terms of crude protein $(P=0.854)$, ether extract $(P=0.203)$, as well as AMEN $(P=0.810)$. Whereas, the weights of selected gastrointestinal tract segments were reduced, i.e. jejunum $(P<0.001)$, ileum $(P<0.001)$ by $H$. illucens fat addition to the broiler diets. Present data suggest that soybean oil may be replaced by $H$. illucens without negative effects on the growth performance during $35 \mathrm{~d}$ rearing. This work was financed by the National Centre for Research and Development within the LIDER VII Programme No. LIDER/5/0148/L-7/15/NCBR/2016, titled 'IN OIL project: An innovative method for bio-conversion of byproducts from food processing industry'. 


\title{
Black soldier fly larvae as a substitute for soybean in the diets of laying hens
}

\author{
M. Heuel ${ }^{1 *}$, C. Sandrock ${ }^{2}$, A. Mathys ${ }^{3}$, M. Gold ${ }^{3,4}$, C. Zurbrügg ${ }^{4}$, M. Kreuzer ${ }^{1}$ and M. Terranova ${ }^{1}$ \\ ${ }^{1}$ ETH Zurich, Universitätstr. 2, 8092 Zurich, Switzerland; ${ }^{2}$ FIBL, Ackerstr. 113, 5070 Frick, Switzerland; ${ }^{3}$ ETH Zürich, \\ Schmelzbergstr. 9, 8092 Zurich, Switzerland; ${ }^{4}$ Eawag/Sandec, Überlandstr. 133, 8600 Dübendorf, Switzerland; \\ maike.heuel@usys.ethz.ch
}

The use of soybean as a feed ingredient for livestock is controversially discussed worldwide, since cultivation and transport may have negative environmental impacts. Thus, interest in integrating insects into livestock nutrition as a potentially more sustainable substitute for soybean is very important, but has been poorly researched so far. This study aimed to examine the feeding value of two origins of black soldier fly (BSF) larvae grown on two different substrates (A and B) compared to soybeanbased diets. For this purpose, 50 Lohmann Brown Classic hens (40 weeks of age) were fed five different diets for 7 weeks. The hens were randomly allocated to the diets and kept individually in enriched cages under controlled climate conditions. To determine whether the protein value of the insect material is comparable to that of soybean, the crude protein content of four of the diets (SS-, AA-, AB-, BB-) was set to a level (<15\% in DM) which was slightly deficient (recommended: $>16 \%)$. These diets were opposed to a control diet (SS) covering requirements. Both SS and SS- contained 15\% soybean cake and 3\% soybean oil, but SS- did not contain sunflower cake. In the other diets the soybean-based feeds from SS- were replaced by defatted BSF larval meals and fats. Insect material A was produced on a mixture of fruits, brewer's grain and pasta production waste, material B was produced on wheat bran, French fries and cereal milling by-products. Diet AA- contained 15\% of larval meal A and 2\% larval oil A, AB- was based on 15\% larval meal A and 2\% larval fat B, and BB-contained 15\% of larval meal B (rich in residual fat). Feed intake, laying performance and egg weight were measured daily. The hens were weighed weekly. Over a period of six days all eggs per hen were collected and analysed for different egg quality traits. The performance of the hens did not differ significantly between the five treatments within the feeding period. Average feed intake was around $118 \mathrm{~g} /$ day, laying percentage 95\%, egg weight $65 \mathrm{~g}$ and feed conversion efficiency $1.9 \mathrm{~g}$ egg/ $\mathrm{kg}$ feed. Also, egg quality was neither affected by the use of insects nor the apparent protein deficiency. Average shell thickness was $0.4 \mathrm{~mm}$, yolk height 17 $\mathrm{mm}$ and shell breaking strength $50 \mathrm{~N}$. Overall, the similar performance of the hens at a very high production level and the comparable egg quality showed that soybean can be completely replaced by BSF meal and fat. The results also indicated that the recommendations for protein supply of layers may be set too high.

\section{A preliminary study on insect full-fat meals preferences in guppy (Poecilia reticulata)}

\author{
M. Rawski ${ }^{1}$, J. Kowalska ${ }^{1}$, N. Homska ${ }^{1}$, Z. Mikołajczak²", J. Mazurkiewicz ${ }^{1,3}$, A. Józefiak ${ }^{4}$, B. Kierończyk ${ }^{2}$, \\ R. Wachowiak ${ }^{2}$ and D. Józefiak ${ }^{2,3}$ \\ ${ }^{1}$ Division of Inland Fisheries and Aquaculture, Institute of Zoology, Poznan University of Life Sciences, Wojska Polskiego \\ 71c, 60-625 Poznań, Poland; ${ }^{2}$ Department of Animal Nutrition, Poznan University of Life Sciences, Wotyńska 33, 60-637 \\ Poznań, Poland; ${ }^{3}$ HiProMine S.A.,Poznańska 8, 62-023 Robakowo, Poland; ${ }^{4}$ Department of Preclinical Sciences and Infectious \\ Diseases, Poznan University of Life Sciences, Wotyńska 35, 60-637 Poznań, Poland; zuzanna.mikolajczak@up.poznan.pl
}

In companion animal nutrition one of the most important factors affecting feed market trends is nutritional preferences of pets, owners observations as well as the sustainability of the product. Due to that pet foods should be based on well accepted by animals and owners feed ingredients; a free choice feeding experiment is reported herein. In a ten-day-long experiment using the free choice model were applied. A group of 60 sexually-mature Poecilia reticulata. The animals were randomly divided into 4 identical experimental tanks with a capacity of 32 litres $(30 \times 30 \times 35 \mathrm{~cm})$ using 15 individuals in each. The fish were subjected to a 5 -day preliminary period, then they were held observation by 5 days of food preferences. As the assessed components, full-fat insect meals were used, with pork gelatine and fish meal as the control treatments. The species of insects used for meals preparation were: Madagascar cockroach (Gromphadorhina portentosa), superworm (Zophobas morio), black soldier fly (Hermetia illucens). The applied components were solidified using pork gelatine in a ratio of 3:2. To avoid the effect of neophobia the results from the first day of experiments were excluded from the final results of the experiment. In the following days the attractivity of feeds was observed during periods of $1,2,3,4$ and 5 minutes after offering them to animals. The experiment showed that the most accepted, among all offered components was superworm. The fish interest in Madagascar cockroach, superworm, black soldier fly meals were comparable to fish meal. Pork gelatine was not assessed as attractive for guppies. P. reticulata have high food selectivity, among of the tested feed components Zophobas morio full fat meal can be recommended as an attractive ingredient to aquarium fish feeds. This work was supported by several sources: Poznań University of Life Sciences; TEAM TECH/2016-2/11-0026, a project entitled: Insects as novel protein sources for fish and poultry, financed by Foundation of Polish Science (POIR 4.4); and the National Centre for Research and Development, no POIR.01.01.01-00-0828/15, entitled: InnSecta: innovative technology of feedstuffs production based on insect biomass. 


\section{How do hydrolyzed insect meals affect sea trout (Salmo trutta trutta) microbiota?}

\section{Z. Mikołajczak ${ }^{1 *}$, M. Rawski ${ }^{2}$, J. Mazurkiewicz ${ }^{1,3}$, A. Józefiak ${ }^{4}$, B. Kierończyk ${ }^{1}$ and D. Józefiak $^{1}$}

${ }^{1}$ Department of Animal Nutrition, Poznan University of Life Sciences, Wotyńska 33, 60-637 Poznań, Poland ${ }^{2}$ Division of Inland Fisheries and Aquaculture, Institute of Zoology, Poznan University of Life Sciences, Wojska Polskiego 71c, 60-625 Poznań, Poland; ${ }^{3}$ HiProMine S.A.,Poznańska 8, 62-023 Robakowo, Poland; ${ }^{4}$ Department of Preclinical Sciences and Infectious Diseases, Poznan University of Life Sciences, Wotyńska 35, 60-637 Poznań, Poland; zuzanna.mikolajczak@up.poznan.pl

Insect meals are a promising source of protein and fat for aquafeeds. It should be underlined that, dietary protein and fat composition have a crucial impact on microbiota composition and the beneficial of harmful relation among the host and microbial populations. Moreover, insects are being widely discussed as a source of antimicrobial peptides (AMP) which exhibit activity against pathogens, they contain chitin also which is considered a prebiotic compound. The 60 day experiment was performed on sea trout (Salmo trutta $\mathrm{m}$. trutta) fingerlings. The animals were fed with three experimental diets: control diet (CON) with fishmeal (25\%) as the only source of animal protein; TMD: 14.5\% of fishmeal and 10\% of hydrolysed Tenebrio molitor meal; and ZMD: 14\% of fishmeal and 10\% of hydrolysed Zophobas morio meal. At the end of the trial, the fish were euthanised, the samples of the gastrointestinal content were collected and immediately stored at $-80^{\circ} \mathrm{C}$ for fluorescent in situ hybridisation. The analysis was performed with the use of oligonucleotide probes selected from the literature labelled with fluorochromes. The numbers of detected bacteria are expressed in colony-forming units/g of digesta (cfu/ml). The use of both insect meals affected gastrointestinal microbiota by decreasing bacterial numbers. The inclusion of $T$. molitor meal lowered the concentration of Carnobacterium spp. and Lactobacillus group. Z. morio inhibited Aeromonas spp., Carnobacterium spp. and Lactobacillus group. The total number of bacteria, as well as Bacillus spp., did not exhibit any significant differences among treatments. The results show the possibility of fish microbiota modification by the use of full-fat insect meals. The lowered numbers of bacterial groups may be the effect of both inhibiting properties of antimicrobial peptides, as well as the prebiotic features of chitin. The result which should be underlined is the decrease of Aeromonas spp. population - commonly known fish pathogen in population in ZMD treatment. The results of the study suggest that insect meals may be used not only as protein and fat sources but as a functional ingredient also. The antimicrobial properties of Z. morio and T. molitor meals suggest that they should be considered for further use in aquaculture. 


\title{
Insect production systems/genetics, nutrition, physiology, health \& ethics of edible insects
}

\section{Gastrointestinal evacuation in black soldier fly larvae}

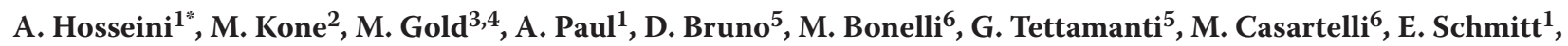
A. Mathys ${ }^{3}$, G. Vandenberg ${ }^{2}$ and M.H. Deschamps ${ }^{2}$

${ }^{1}$ Protix, Industriestraat 3, 5107 NC Dongen, the Netherlands; ${ }^{2}$ Université Laval, Dép. Sciences Animales, Pav. Paul-Comtois, 2425 rue de l'Agriculture, G1V 0A6, Québec (Qc), Canada; ${ }^{3}$ ETH Zurich, Sustainable Food Processing, Department of Health Science \& Technology, Institute of Food, Nutrition \& Health, Zurich, Schmelzbergstrasse 9, 8092 Zurich, Switzerland;

${ }^{4}$ Eawag, Swiss Federal Institute of Aquatic Science and Technology; Department of Sanitation, Water and Solid Waste for Development (Sandec), Überlandstrasse 133, 8600 Dübendorf, Switzerland; ${ }^{5}$ University of Insubria, Department of Biotechnology and Life Sciences, via J.H. Dunant 3, 21100 Varese, Italy; ${ }^{6}$ University of Milan, Department of Biosciences, via Celoria 26, 20133 Milan, Italy; sali@protix.eu
\end{abstract}

In some industrial post-harvest processing systems of black soldier fly (BSF) (Hermetia illucens), the larvae are minced and homogenised for protein and fat extraction. The quality of the resulting products can be affected by the gastrointestinal content of the insect. This content is poor in nutrients and rich in microbes and digestive enzymes. Post-harvest starvation has been suggested as a gastrointestinal evacuation (GE) method for BSF larvae, however, no direct evidence is available for GE and improvement of the quality of larvae after starvation. In the present study, we determined the GE dynamics through dissection of BSF larvae kept for $72 \mathrm{~h}$ under different temperature and substrate conditions after harvest. Different GE patterns were observed, depending on the environmental condition after harvest. At low temperature $\left(10^{\circ} \mathrm{C}\right)$, GE was not completed (>90\%). The most complete GE was observed after $6 \mathrm{~h}$ in water and after $48 \mathrm{~h}$ under fasting. Shortening the pre-slaughter period is important to avoid the decline of nutritional and microbial qualities of larvae. Interestingly, further investigations indicated improvements in the nutritional, colour, and microbial qualities of the larvae after specific GE treatments. These larvae showed higher crude protein and fat, reduced microbial counts, lower wet weight, lighter puree colour, and no change in their dry weight, ash, and fibre contents compared to larvae after conventional fasting. Broadly, results indicate that it may be beneficial to add an optimised degutting method after harvest to improve the quality of larvae for processing. 


\title{
How the absence of microbiota in black soldier fly (Hermetia illucens) larvae affects gene expression
}

\author{
L. Auger ${ }^{1 *}$, M.H. Deschamps ${ }^{2}$, S. Bouslama ${ }^{1}$, G. Vandenberg ${ }^{2}$ and N. Derome ${ }^{1}$ \\ ${ }^{1}$ Université Laval, Department of Biology, Institute for Integrative and Systems Biology (IBIS), Derome Laboratory, \\ Québec, QC, Canada; ${ }^{2}$ Université Laval, Department of Animal Science, Vandenberg Laboratory, Québec, QC, Canada; \\ laurence.auger.1@ulaval.ca
}

Black soldier fly larvae (BSF, Hermetia illucens) has gained much attention in the last few years for its use as biowaste recycler as well as a new source of animal proteins. An increased interest has been shown toward the microbiota associated with BSF for the roles the microorganisms have in host development, behaviour, metabolism and immune system. However, the functions and dynamics of the microbiota, the effect they have on insect health and growth performance remain vastly unknown. This study endeavours to clarify the role of the microbiota on BSF ontogeny by exploring genomic expression during larval development in axenic conditions (i.e. absence of microorganisms). We hypothesised that the absence of the microbiota will be associated with reduced expression of genes linked to digestion, growth and immunity. During transcriptome analysis, we looked at differentially expressed transcripts using DESeq2 to compare conventional and axenic larvae (reared on BHI culture growth media, $28^{\circ} \mathrm{C}, 70 \% \mathrm{RH}$ and $12 \mathrm{~L}: 12 \mathrm{D}$ photoperiod) at day 4,8 and 10 post-hatching (sample $\mathrm{n}=6 /$ replica, each condition $=6$ replica). To produce axenic larvae, eggs were dipped in $2.5 \%$ active chlorine, then in $70 \% \mathrm{EtOH}$ followed by in PBS 1X (3 min/solution) and hatched in sterile ventilated flask. Larval length $( \pm 0.5 \mathrm{~mm})$ revealed that the rearing substrate resulted in poor growth (axenic $=1.5 \pm 0.5 \mathrm{~mm}$; conventional $=2 \pm 0.5 \mathrm{~mm}$ at day 10 ) compared to the usual Gainesville substrate (control $=18 \pm 1.5 \mathrm{~mm})$. No significant difference was observed between the axenic and conventional groups. Gene expression is currently being analysed with RSEM and annotated to characterise variation in time and underline the implicated gene functions and involved pathways in BSF development in presence $v s$ in absence of microbiota. This study explores the effect of microbiota on host expression during development and is paving the way to future researches aiming to modulate BSF microbiota for rearing optimisation, thus facilitating industrial production.

\section{Morphofunctional adaptation of Hermetia illucens larval midgut to rearing substrates with different nutritional quality}

\author{
D. Bruno ${ }^{1}$, M. Bonelli ${ }^{2}$, M. Brilli ${ }^{2}$, N. Gianfranceschi ${ }^{2}$, S. Caccia ${ }^{3}$, M. Casartelli ${ }^{2}$ and G. Tettamanti ${ }^{1 *}$ \\ ${ }^{1}$ University of Insubria, Department of Biotechnology and Life Sciences, via J.H. Dunant 3, 21100 Varese, Italy; ${ }^{2}$ University \\ of Milano, Department of Biosciences, via Celoria 26, 20133 Milano, Italy; ${ }^{3}$ University of Napoli Federico II, Department \\ of Agricultural Sciences, via Università 100, 80055 Portici, Italy; gianluca.tettamanti@uninsubria.it
}

Black soldier fly (Hermetia illucens) larvae (BSFL) are increasingly used for waste management purposes because of their extraordinary ability to feed on a variety of waste organic matter and convert this material into valuable products. Although nutrient composition of the rearing substrate can affect larval performances and the bioconversion process, the astonishing adaptability of this insect to dietary substrates, without any dramatic impact on its development, strongly suggests that BSFL can finely and profitably regulate nutrient intake and post-ingestion processes to match their nutritional requirements. In the present study we investigated if and how the midgut of BSFL, which is involved in the digestion and absorption of nutrients, sets in motion post-ingestion responses to compensate variations in nutrient composition of the rearing substrate. To this purpose, we compared insects grown on a nutritionally balanced diet for dipteran larvae and a nutritionally poor diet that mimics fruit and vegetable waste composition, a substrate produced in large amount worldwide, whose use is allowed in the European Union for the mass rearing of insects that are used for the production of fish feed. Our morphofunctional characterisation of the midgut responses, supported by a transcriptomic analysis, shows a diet-dependent adaptation process of the midgut that allows the larva to fully exploit the unbalanced diet, with minor effects on the growth performance. Besides differences in cell morphology, activity of digestive enzymes, and accumulation of long-term storage molecules, midgut cells of larvae grown on fruit and vegetable waste show differential expression of genes coding for digestive enzymes, transport proteins, and immune factors. Our data demonstrate that the functional plasticity of the midgut has a key role in the ability of BSF larvae to grow and develop on substrates with low nutrient content. 


\title{
Growth, performance and larval composition of Alphitobius diaperinus on agri-food side-streams
}

\author{
N. Gianotten ${ }^{1 *}$, L. Soetemans ${ }^{2}$ and L. Bastiaens ${ }^{2}$ \\ ${ }^{1}$ Protifarm, Harderwijkerweg 141B, 3852 AB Ermelo, the Netherlands, ${ }^{2}$ VITO, Boeretang 200, 2400 Mol, Belgium; \\ n.gianotten@protifarm.com
}

Larvae of the Alphitobius diaperinus or the lesser mealworm (LMW) are regarded as one of the insects which is fit for human consumption. In Ermelo, at Protifarm, this insect species is reared on large scale. Feed for the LMW is, just like other traditional livestock: (1) a large part of the production cost; and (2) has an enormous impact on the sustainability of the rearing system. Side-streams from the agri-food sector are potential candidates as ingredients in insect feed. In addition, when side-streams are used as replacement of cereals, the feed-food competition is also reduced. Within the BBI-InDIRECT project (www.bbiindirect.eu), six agri-food side-streams were employed (wheat middlings, DDGS, rapeseed meal, corn gluten feed, rice bran and brewery grains) in different mixtures. The effect of 29 diets (single side-streams or mixtures) on larval performance was investigated in an industrial research set-up. Yield per tray, individual larval weights and bioconversion efficiencies were used as parameters for larval performance. The influence of the type of side-stream and the presence of a second or third side-stream in the ration was evaluated. In general, mixtures of side-streams resulted in better growth, and the best results were obtained with a wheat middlings - rapeseed meal - brewery grains ration based on larval performance and economic considerations. Next to larval growth, the effect of feeding LMW larvae with these side-streams on the nutritional composition of the larvae was investigated. LMW larvae are intended to be processed into ingredients for human food for which the amount and profile (composition) of both protein and fat is important. A possible higher variability in composition of the insect feed when using side-streams might result in a more variable end product (= larvae) which is the starting product for food applications. Changes in composition can be regarded as positive (more protein, better amino acid profile) or as negative (more variation in starting product for food applications when the diet is not stable year-round). In general, the composition (nutrient profile = type of compounds) of the larvae was found to be relatively stable, although some variations in absolute concentration were observed.

\section{The yellow mealworm genome: a resource for all}

\author{
C.J. Picard ${ }^{1 *}$, T. Erikkson ${ }^{1,2}$, A.A. Andere ${ }^{1}$, H. Kelstrup ${ }^{2}$ and V.J. Emery ${ }^{2}$ \\ ${ }^{1}$ Indiana University Purdue University Indianapolis, Department of Biology, 723 W Michigan Street, Indianapolis, IN \\ 46202 USA; ${ }^{2}$ Beta Hatch Inc., 200 Titchenal Rd, Cashmere, WA 98815, USA; cpicard@iupui.edu
}

The yellow mealworm (Tenebrio molitor) is one of the most produced insects for food and feed globally. Despite being commercially produced, little is known about one of its most basic properties - its genome and how this genome dictates its phenotypes. The phenotype of a particular insect is integral to its use in mass production: one must understand how quickly it can grow, how big it can get, how fecund it can remain, and how resilient it is to perturbations in its environment all in an effort to optimally produce safe and reliable insects for food and feed. We generated a genome of a strain of the yellow mealworm using linked-read technology (10X Genomics). This technology is a combination of short read sequencing technology that is accurate and reliable with long-read capabilities, resulting in a preliminary draft genome that encompasses $\sim 90 \%$ of its gene content according to conserved homologous database queries (with more than 20,000 genes assembled). The genome (312 $\mathrm{Mb}$ ), with more than half its genome present in contigs that are 40,000 bp or longer, allows us to immediately understand the gene structures, but also to delve into regulatory regions in the genome in the future. Changes in these regulatory sequences are what will provide the greatest value to the industry when correlated to phenotypic properties of the insects, leading to a more efficient selection of optimal insects for mass production. Currently, this is often done using selective breeding which has the potential to introduce some troublesome (piggybacked) traits that have the potential to offset its entire production value. This resource will allow all in the industry to compare and contrast their strains without needing to sequence whole genomes, and allow the industry to move forward at the same pace as other agriculturally important products. 


\title{
Evaluation of the suitability of Greek agricultural by-products for the rearing of Tenebrio molitor
}

${\text { C.I. } \text { Rumbos }^{1 *} \text {, D.G.A.B. Oonincx }}^{2}$, M. Vrontaki ${ }^{1}$, A. Asimaki ${ }^{3}$, E. Mente ${ }^{3}$, I.T. Karapanagiotidis ${ }^{3}$ and C.G. Athanassiou $^{1}$

${ }^{1}$ Laboratory of Entomology and Agricultural Zoology, Dept. of Agriculture, Crop Production and Rural Environment, University of Thessaly, Volos, Greece; ${ }^{2}$ Animal Nutrition Group, Animal Sciences Group, Wageningen University, Wageningen, the Netherlands; ${ }^{3}$ Aquaculture Laboratory, Department of Ichthyology and Aquatic Environment, University of Thessaly, Volos, Greece; crumbos@uth.gr

The evaluation of alternative feeding substrates for the mass-rearing of insects is one of the main current research priorities of the insect sector. These substrates should be preferably by-products or waste-streams that can be upcycled by insect bioconversion, with low cost, and should ideally be locally produced. In Greece, various by-products are produced as a result of several agro-industrial processes and are currently either used as low-cost feed for livestock animals or discarded as waste. In this study, we evaluated the suitability of 11 Greek agricultural by-products (sugar beet pulp meal, sunflower meal, cotton cake, cotton seed meal, by-products of the seed cleaning process of barley, oats, pea and vetch) for the rearing of larvae of the yellow mealworm, Tenebrio molitor. In a first series of bioassays, we evaluated the suitability of each by-product alone. Briefly, plastic cylindrical vials were filled with $20 \mathrm{~g}$ of each substrate and then 20 newly emerged T. molitor larvae were introduced into the vials. Larvae were allowed to feed undisturbed ad libitum at $26{ }^{\circ} \mathrm{C}$ and $55 \%$ relative humidity for a 4-week period. After this interval, larval survival and weight were recorded. Afterwards, larvae were returned to their corresponding vials, provided with new substrate $(20 \mathrm{~g})$, and returned to the growth chamber for another 4-week period. This process was repeated for two more 4-week periods completing a total of three 4-week periods of development. Wheat bran served as a 'control diet'. In a second series of bioassays, the suitability of mixtures of each by-product with wheat bran (1:1) was evaluated, following the experimental design described above. Finally, based on the results of the previous two series of bioassays and the nutritional composition (protein, lipid, carbohydrate content, ash and energy) of the by-products, isonitrogenous diets based on the best performing by-products were designed and evaluated. According to our results, the cereal by-products tested, i.e. barley and oats by-products, were highly suitable for the rearing of T. molitor larvae and could be used as the core ingredient of $T$. molitor diets. Our findings contribute to implementing insect farming in the circular economy at a local level. This work was supported by a postdoctoral scholarship program implemented by University of Thessaly and funded by Stavros Niarchos Foundation.

\section{Farming fecund crickets: fruitful female fertility after feeding crickets royal jelly}

\author{
M.J. Muzzatti, H.A. MacMillan and S.M. Bertram \\ Carleton University, 1125 Colonel By Dr, Ottawa, ON K1S 5B6, Canada; mattmuzzatti@cmail.carleton.ca
}

Insects are a sustainable and nutritious alternative protein source, and crickets are an economically important insect in the North American entomophagy industry. A primary goal of agricultural research is to increase yield. Increased body size in cricket farms is desired, but we have little knowledge on how to do it at such a large scale. Diet supplementation using honey bee royal jelly is a potential solution, as there is evidence that royal jelly enhances body size of other insect species including a single orthopteran. The mechanisms behind how royal jelly does this remain unclear. To determine the effect of royal jelly on a farmed cricket species, 96 Gryllodes sigillatus were obtained from a local Canadian cricket farm, individually housed, and split into two treatment groups: half were reared on a commercially available cricket diet, while the other half were reared on the same diet mixed with $15 \% \mathrm{w} / \mathrm{w}$ fresh royal jelly. Body size and mass measurements were taken weekly for six weeks (approximate time to adulthood). We discovered a female-only effect of royal jelly on G. sigillatus: females fed the royal jelly diet grew to be $21 \%$ heavier, and this effect was driven by significantly longer abdomens containing $66 \%$ more eggs each compared to those fed the basal diet. We are now replicating this experiment in a simulated farm environment to determine whether increased female fertility from royal jelly persists in high-density rearing environments like an active commercial farm. 


\title{
Deuterium labelling of fatty acids in Hermetia illucens larvae
}

\author{
B. Hoc ${ }^{1}$, M.L. Fauconnier ${ }^{2}$, G. Purcaro ${ }^{3}$, G. Lognay ${ }^{1}$, F. Francis ${ }^{1}$ and R. Caparros Megido ${ }^{1 *}$ \\ ${ }^{1}$ Gembloux Agro-Bio Tech, University of Liège, Functional and Evolutionary Entomology, TERRA, Passage des Déportés 2, \\ 5030 Gembloux, Belgium; ${ }^{2}$ Gembloux Agro-Bio Tech, University of Liège, Chemistry of Natural Molecules, TERRA, Passage \\ des Déportés 2, 5030 Gembloux, Belgium; ${ }^{3}$ Gembloux Agro-Bio Tech, University of Liège, 3Analytical Chemistry, TERRA, \\ Passage des Déportés 2, 5030 Gembloux, Belgium; r.caparros@uliege.be
}

Black soldier fly larvae (BSFL, Hermetia illucens L. 1758) are increasingly produced as animal feed.. In Europe, as BSFL should be used for fish production, it is interesting to characterise their fatty acid (FA) composition but also to understand the underlaying biochemical pathways.. In the present study, 3 batches of 50 larvae $(n=3)$ were produced on 4 different diets: (1) $100 \%$ chicken feed with $40 \% \mathrm{H}_{2} \mathrm{O}$ (CF); (2) $40 \%$ chicken feed and $60 \%$ of flax cake with $40 \% \mathrm{H}_{2} \mathrm{O}$ (FL); (3) CF with $40 \%$ deuterated water $\mathrm{D}_{2} \mathrm{O}$ (CFD); and (4) FL with $40 \% \mathrm{D}_{2} \mathrm{O}$ (FLD). Growing parameters as developmental time (DT) and individual weight (IW) were collected throughout BSFL development. Before pupation, BSFL were collected and, following a Folch extraction, fatty acid methyl esters of each larval batches were analysed by gas chromatography coupled with mass spectrometry. The DT and LW of BSFL were significantly impacted by the presence of deuterated water in diets (CFD: 20 days and $121.27 \pm 6.46^{\mathrm{C}} \mathrm{mg}$; FLD: 28 days and $\left.116.57 \pm 6.56^{\mathrm{C}} \mathrm{mg} ; \mathrm{F} 12=151.40 ; P<0.001\right)$. Concerning the two other diets, the DT were equal (9 days) while the $\mathrm{LW}$ was greater for CF $\left(251.16 \pm 4.47^{\mathrm{a}} \mathrm{mg}\right)$ than for FL $\left(200.04 \pm 6.17^{\mathrm{b}} \mathrm{mg}\right)$. BSFL fed with FL show higher levels of $\alpha$-linolenic acid (C18:3n3) than BSFL fed with CF (respectively $0.70 \pm 0.03$ and $5.64 \pm 1.38 \%$ ). The analysis of FA from BSFL fed with deuterated diets shows only a deuterated form of $\mathrm{C} 10: 0$ and $\mathrm{C} 12: 0$ and a partial percentage of deuterated C14:0, C16:0, C16:1, C18:1n9 and C18:2n6. The complete deuteration of these 3 FA were expected as they were not present in diets and confirm the reliability of the method. When fatty acids are found in the two forms, it appears that some of these were produced via biosynthesis pathways while another proportion of the FA was accumulated from the diet. Proportions of C12:0 were reduced in BSFL fed with deuterated diets in favour of C18:1n9 (approximately from 20\% in 'normal' diets to 30\% in deuterated diets). This trend has been verified in other tests carried out with flax-based diet in our laboratory, perhaps due to a better assimilation of this FA from flax-based diet by BSFL. Finally, no deuterated C18:3n 3 were found in BSFL fed with FLD confirming that the BSFL are not able to produce this FA but can simply accumulate it. It will be interesting to incorporate only one deuterated fatty acid and to monitor its use by the BSFL.

\section{Effect of black soldier fly density in oviposition cages on egg production}

\author{
T. Kusumawardhani, T. Rahayu, B.M.A. Dortmans" and C. Zurbrügg \\ Eawag - Swiss Federal Institute of Aquatic Science and Technology, Department of Sanitation, Water and Solid Waste for \\ Development (Sandec), 8600 Dübendorf, Switzerland; bram.dortmans@eawag.ch
}

The black soldier fly (BSF) rearing system realised by the FORWARD project in Sidoarjo, Indonesia uses pupation (dark) cages and oviposition (love) cages. Flies emerge from pupae inside a dark cage and are then harvested into love cages by connecting the cages and attracting the flies using a light source. The current setup utilises fly densities of 7,000-8,000 flies in each love cage $(70 \times 70 \times 150 \mathrm{~cm})$. When considering limited space at small decentralised BSF facilities, it is a key objective to optimise egg productivity in relation to fly density in love cages. This trial tested thirteen different fly densities in love cages. These densities ranged from 3,000-15,000 flies with increments of 1000 and a triplet setup for each density setting. Prepupae, with weights varying between $113 \pm 4.7$ and $164 \pm 20.7 \mathrm{mg}$, were added into pupation crates of which 16 were placed inside each dark cage containing a number of prepupae ranging from 120,000-320,000. After the love cages were stocked with the defined density of flies, they were placed in a shaded area, which received abundant indirect natural light. Three batches of 20 flies were randomly extracted from each love cage. For each sample of 20 flies, the sex ratio and the average weight were investigated. Preliminary sampling results show that on average, flies weighed $62.5 \pm 2.6 \mathrm{mg}$ each at point of harvesting. Each love cage included a container with attractant substrate (a mixture of dead flies, treatment residue and fermented fruit water), wooden egg media, a shade box and a water container. Relative humidity and temperature were recorded both in the dark cage room and the surrounding area of the love cages during the complete duration of the trial. After four days, the eggs produced were harvested and weighed and each love cage was dismantled. The tested range could show that with higher density of flies in love cages, a twofold increase of egg production per square meter can be realised compared to the current setup, thus reducing the space footprint and labour workload. With the triplet setup for each tested density it was further possible to obtain information on how the sex ratio impacts on the egg production performance. 


\title{
Effect of temperature on life-history parameters and production performances of the common housefly, Musca domestica
}

\author{
L. Francuski ${ }^{1 *}$, W. Jansen ${ }^{2}$ and L.W. Beukeboom ${ }^{1}$ \\ ${ }^{1}$ Groningen Institute for Evolutionary Life Sciences, University of Groningen, P.O. Box 11103, 9700 CC Groningen, the \\ Netherlands; ${ }^{2}$ Amusca B.V.Bennebroekerdreef 7, 2121 CL Bennebroek, the Netherlands; l.francuski@rug.nl
}

Musca domestica, the common housefly, is increasingly considered as a new, alternative protein source for animal nutrition and biowaste conversion. Easy rearing requirements and the ability of housefly larvae to grow on many different substrates make them useful to turn waste (e.g. manure) into a valuable biomass. Still, industrialisation of housefly faces numerous challenges and both fundamental (understanding of housefly biology) and applied (improvements on housefly production method) research is needed. We investigated life-history traits relevant for mass rearing in three European housefly strains (originated from Spain, Italy and the Netherlands) at two temperatures; 25 and $32{ }^{\circ} \mathrm{C}$. All three strains responded to $32{ }^{\circ} \mathrm{C}$ with shortened duration of preoviposition period, laying phase and overall longevity. Moreover, wing/body size, lifetime egg production, mating activity and sperm quantity and viability declined at higher temperature. In contrast, number of clutches laid per female, hatchability, percentage of pupation, adult emergence rate and wing shape were unaffected by temperature. Although females laid on average $20 \%$ fewer eggs at $32{ }^{\circ} \mathrm{C}$, the overall efficiency of the rearing process was increased. The combination of shorter sexual maturation period and higher daily egg-laying rate resulted in reaching $50 \%$ of total egg production in only six days at $32^{\circ} \mathrm{C}$, compared to $13-14$ days at $25^{\circ} \mathrm{C}$. Our results point at beneficial effects on production performances of $M$. domestica at higher than $25^{\circ} \mathrm{C}$ and indicate that $32^{\circ} \mathrm{C}$ is well suited for efficient housefly rearing.

\section{Genetic variation in qualitative/quantitative lipid content of three strains of housefly larvae at different temperatures}

\author{
F. Boatta ${ }^{1 *}$, W. Jansen ${ }^{2}$, L.W. Beukeboom ${ }^{3}$ and J. Ellers ${ }^{1}$ \\ ${ }^{1}$ Department of Ecological Science, Vrije Universiteit Amsterdam, De Boelelaan 1085, WEN Building, 1081 HV, Amsterdam, \\ the Netherlands; ${ }^{2}$ Amusca B.V., Bennebroekerdreef 7, 2121 CL Bennebroek, the Netherlands; ${ }^{3}$ Groningen Institute for \\ Evolutionary Life Sciences, University of Groningen, P.O. Box 11103, 9700 CC Groningen, the Netherlands; f.boatta@vu.nl
}

There is a growing interest in the use of insect larvae as an alternative source of proteins for the livestock sector. A species which has so far been underappreciated for its application as feed is the common housefly, Musca domestica. The balanced amino acid profile of this species, its fast life cycle, straightforward production line, and its ability to convert many biological materials into valuable products make $M$. domestica a good candidate for industrial applications. A potential drawback of the species when applied as animal feed is the high relative lipid content of the larvae. Despite the lipids stored during the larval phase represent an essential energy source, a high lipid content also limits the use of insect larvae as feed given its negative health effect on livestock. Lipid content and composition of insects reflects a combination of genetic and environmental factors such as diet. Differences in lipid accumulation may occur between strains derived from different regions, due to the cosmopolitan distribution and phenotypic plasticity of the common housefly. Moreover, strains may differ in the lipid composition depending on rearing temperature as a result of climate adaptation. In this study we characterised the qualitative and quantitative lipid profile of the late developmental stages (third instar) of the housefly larvae originated from three European locations (Italy, Spain, and the Netherlands) to identify a strain with favourable lipid accumulation and lipid composition characteristics. Additionally, we also characterised the lipid profile of the larvae at two different temperatures to evaluate if the three selected strains responded differently to a temperature close to the upper tolerance limit of the species. The results of this study provide insight into the variation in lipid metabolism in the common housefly and its adaptation to high temperatures; it also discusses the importance of selecting a suitable strain with desired characteristics applicable into industrial settings. 


\title{
Fecundity and larval growth of Tenebrio molitor reared on differently composed diets of similar nutritional composition
}

\author{
S. Naser el Deen ${ }^{1,2}$, V. Verrastro ${ }^{2}$, F. Lamaj ${ }^{2}$, L. al Bitar ${ }^{1,2}$ and F. Baldacchino ${ }^{3}$ \\ ${ }^{1}$ Parthenope university of Naples, Environment, Resources and Sustainable Development Department, 80133 Napoli (NA), \\ Italy; ${ }^{2}$ CIHEAM-IAMB Mediterranean Agronomic Institute of Bari, 70010 Valenzano (BA), Italy; Italian National Agency \\ for New Technologies, Energy and Sustainable Economic Development (ENEA), Trisaia Research Center, Department \\ SSPT-BIOAG-PROBIO, 75026 Rotondella (MT), Italy; somaya.nasereldeen@uniparthenope.it
}

The artificial diet of Tenebrio molitor $(\mathrm{L})$ has been a focal point of many studies that aimed to design the best diet combination. Recent studies have been focusing on the use of by-products and their efficiency as diets for T. molitor rearing. The rearing was successful on a wide range of by-products-based diets. Feed has a profound impact on the lifespan, growth performance, fecundity and nutritional composition of insects like T. molitor. Fecundity is one of the traits that are highly influenced by the diet's composition, especially by the macronutrients. This work aims to study the effect of 8 different diets on the weight and fecundity of females and on their progeny weight: larval weight. Wheat bran, brewer's yeast, spent grain and bread remains were used to make up the diets that were composed of a different inclusion percentage of each by-product. All the diets had narrow range of differences in nutritional composition, mainly macronutrients, although different by-product types. The diets were designed in a way to avoid the effect of different nutritional compositions and study the effect of the by-products type only. Results showed that the difference in female's weight during the egg-laying period under different rearing diets, was not significant. Moreover, the female's fecundity which is measured by the number of larvae, showed no significant difference between the different diets. However, the average larval weight was significantly higher for larvae reared on 2 diets; one composed of wheat bran and brewer's yeast and the second composed of wheat bran, brewer's yeast and spent grain. The mentioned diets although composed of different by-products, have the same amount of protein and carbohydrates. These results prove that the type of by-products included in the experimental diets affects less the performance of T. molitor compared to the nutritional composition.

\section{Yeasts as a supplement in diets diets fed to mealworms (Tenebrio molitor)}

\author{
L. Hénault-Ethier ${ }^{1,2 *}$, A. Fortin ${ }^{2,3}$, D. Marquis ${ }^{2,4}$, É. Normandin ${ }^{2,5}$, M. Tourancheau ${ }^{6}$, M.H. Deschamps ${ }^{1}$ and \\ G.W. Vandenberg ${ }^{1}$ \\ ${ }^{1}$ Faculté des Sciences de l'Agriculture et de l'Alimentation, Université Laval, Québec (QC) Canada, ${ }^{2}$ TriCycle Inc, Montreal \\ (QC) Canada; ${ }^{3}$ École de Technologies Supérieures, Montréal (QC) Canada; ${ }^{4}$ Center for Interdisciplinary Studies in Society \\ and Culture, Concordia University, Montréal (QC) Canada; ${ }^{5}$ Centre sur la Biodiversité, Université de Montréal, Montréal \\ (QC)Canada; ${ }^{6}$ Lallemand, Montreal(Qc)Canada; louise@tricycle.eco
}

Yeasts are eukaryotic, single-celled fungal microorganisms, encompassing some 1,500 species. Traditionally used in wine, beer and bread production, they are also used in raising sterile fruit flies (Drosophila sp.) to control spoiling, and increasingly used as nutritional supplements in the food industry and in livestock feed. Brewer's yeast alone was found to be insufficient to sustain growth in mealworm (Tenebrio molitor, but an addition of up to 5\% brewer's yeast powder is commonly used to supplement diets. As a probiotic, yeasts supplement in diets is an interesting way to boost the immune system and growth performance of insects. However, compared to traditional insects farming techniques which use nutritionally balanced cereal meals (commonly poultry feed with added vitamins and minerals), the use of agri-food by-products is often constrained by availability of waste. Our studies aims to optimise mealworms by-products diets obtained from circular economy loops in Montreal Canada (spent brewer's grain, off-spec cocoa beans, spent mycelium, fruit and vegetable pulp, grain milling residuals) in conducting small scale feed screening trials. Twenty-one treatments containing $5 \mathrm{~g}$ of various feed (5 replicates) and 10 young larvae $(5 \mathrm{mg}$ ) were monitored for growth over 89 days. Our experiments have shown that feeding mealworms with local by-products results in lower insect growth and final yields compared to commercial poultry feeds. Supplementation with yellow pea, lactoserum or hemp seeds to formulate iso-proteinic diets equivalent to commercial feeds resulted in stunted growth. The addition of B-vitamin complex by supplementing wheat bran or by-products diets with yeast flakes (5\% of feed mass based on dry weight) also increased growth performance in mealworms. Using the above-mentioned experimental design (i.e. 5\% dry weight yeasts supplementation), we are now testing if yeast species (Torula sp. vs Saccharomyces cerevisiae) and status (active, inactive, extracts) as well as ten types of commercially available yeasts (Lallemand, Québec, Canada) and two residual brewer's yeasts can increase growth performance of mealworms. Our work should help to balance insect diets when fed on local by-products and thus, enabling urban circular economy initiative. 


\title{
Nutritional composition and quality of dried earthworm (Lumbricidae) powder
}

\author{
I. Gedrovica \\ Latvia University of Life Sciences and Technologies, 2 Liela Street, 3001 Jelgava, Latvia; ilga.gedrovica@llu.lv
}

The traditional protein sources replacement with new sources of protein that do not have such a negative impact on the environment and resource consumption is a recent years' topicality. As an alternative to traditional protein sources, edible insects are offered. Earthworms (Lumbricidae) could also be used for this purpose. Earthworms have been known in China for centuries as a medicine and nutrient source, but they are used in Europe only to produce vermicompost. Earthworms grown under controlled conditions to increase product safety, then cleaned from vermicompost ( $\tau=36 \mathrm{~h}$ ), blanched several times, dried in a sublimation dryer, and then ground into fine powder was used in the study. The aim of this study was to investigate nutritional composition and quality of dried earthworm powder made from the species Eisenia fetida. The findings of the research showed that earthworm powder is rich in protein and contains all essential amino acids. The protein content of earthworm powder is $74.1 \mathrm{~g} / 100 \mathrm{~g}$ and this is a considerable quantity, because it is almost three times more than the protein content in meat. Moreover, the powder is high in fibres (7.8\%), low in carbohydrate $(<0.2 \mathrm{~g} / 100 \mathrm{~g})$ and sugars, with fat content $9.1 \mathrm{~g} / 100$ g, of which only 2.3 grams are saturated fatty acids, but among unsaturated acids there are omega- 6 , omega-3 and omega- 9 fatty acids. Energy value of earthworm powder is $394 \mathrm{kcal}$ and 1,659 kJ/100 g. The samples tested had moisture content $6 \%$ and $\mathrm{pH}$ 6.8. Microbiological parameters do not exceed the acceptable level for dried dehydrated products. Earthworm powder is similar in appearance to whole grain flour and is easy to use in the preparation of various products. Based on its nutrient content, earthworm powder can be used as nutrient-rich food and could serve for human food also as an ingredient for food product production in the future. The current research has been supported by the European Regional Development Fund under the activity 'Post-doctoral Research Aid', project No 1.1.1.2/VIAA/1/16/190 'New sources of protein for food in Latvia'.

\section{Black soldier fly (Hermetia illucens): potential and challenges of genetic improvement of insects as food and feed}

\author{
S. Dimitrova \\ SD Nasekomo EAD, Mladost 4, Business Park Sofia bldg. 2; fl. 5, 1715 Sofia, Bulgaria; simon.dimitrova@nasekomo.life
}

Humanity is facing problems such as unsustainable utilisation of resources and unstable food security. Timely change of our ways is required. The rise of the insect food and feed industry is coming at the right time offering less environmental impacts in exchange of good quality protein. Insects have the potential to help reach the zero-hunger target, to improve our well-being, to help us be responsible consumers and producers, act in benefit of the climate and the life on land and in water. These are 7 of the 17 Sustainable Development Goals of the United Nations set up to achieve by 2030, underlining the vast potential of the industry. Hermetia illucens known as black soldier fly is one of the main species yielding substantial success. It is globally approved for feed in poultry, aquaculture and pet food. The interest in $H$. illucens comes from its high adaptability to different conditions - it originates from the tropical and warm regions, but it is robust enough to be reared under controlled conditions in other parts of the world. Our team focused on the black soldier fly itself and its genetic capacity. There are multiple unknowns considering the fly physiology and genetics, nevertheless information is being progressively collected starting with studies on suitable rearing conditions and in the last years going to sequencing and transcriptomics data. The black soldier fly genome has been sequenced, unravelling genomic size larger compared to other Dipteran species $(1,102 \mathrm{Mb})$ and significant allele variability giving potential for development of marker-assisted selection. Moreover, there is data on the successful genetic improvement of other insect species such as Bombyx mori L. and Apis mellifera that yielded high producing of the species via hybrid development. Our team believes that lessons from the plant and animal breeding fields are vastly important for the development of a novel optimised $H$. illucens strain. Quantitative genetics tools such as Best linear unbiased predictors and breeding concept such as recurrent selection found in cross-pollinating plants could be adapted and used in black soldier fly breeding to yield commercially important traits- e.g. high fecundity and high protein content. Having in mind the industry background and all scientific data available, our team is focused to evaluate the genetic improvement potential of $\mathrm{H}$. illucens and face challenges such as the adaptation of already established breeding practices and the lack of information and communication between all shareholders. 


\title{
Preliminary in vitro model of the black soldier fly (Hermetia illucens) larvae midgut
}

\author{
M. Gold ${ }^{1,2^{*}}$, J. Egger ${ }^{1,2}$, A. Scheidegger ${ }^{3}$, C. Zurbrügg ${ }^{2}$, D. Bruno ${ }^{4}$, M. Bonelli ${ }^{5}$, G. Tettamanti ${ }^{4}$, M. Casartelli $^{5}$, \\ E. Schmitt ${ }^{6}$ and A. Mathys ${ }^{1}$ \\ ${ }^{1}$ ETH Zurich, Sustainable Food Processing, Schmelzbergstrasse 9, 8092 Zurich, Switzerland; ${ }^{2}$ Eawag - Dept. of Sanitation, \\ Water and Solid Waste for Development (Sandec), Überlandstrasse 133, 8600 Dübendorf, Switzerland; ${ }^{3}$ Eawag - Dept. Systems \\ Analysis, Integrated Assessment and Modelling, Überlandstrasse 133, 8600 Dübendorf, Switzerland; ${ }^{4}$ University of Insubria, \\ Dept. of Biotechnology and Life Sciences, via J.H. Dunant 3, 21100, Varese, Italy; ${ }^{5}$ University of Milan, Dept. of Biosciences, \\ via Celoria 26, 20133, Milan, Italy; ${ }^{2}$ Protix, Industriestraat 3, 5107 NC Dongen, the Netherlands; moritz.gold@hest.ethz.ch
}

In vitro digestion models have been widely applied for farmed animals to predict feed quality and digestibility. Simulation of digestion could have similar applications for the emerging insect farming industry. Static in vitro digestion models are useful because of their simplicity, reproducibility, and low cost. They consider the number of digestive tract regions, pHs, enzymes, temperatures, and residence times. We propose a static in vitro model simulating the digestive tract of black soldier fly larvae (BSFL). As the digestive tract of fly larvae presents similarities to that of humans, the starting point for the model was the INFOGEST international standard human digestion model. Based on the existing knowledge on BSFL biology, the most important organ for digestion is the midgut, composed of three regions varying in pHs, enzyme activities, and feed residence times: anterior, middle, and posterior midgut. Previous research has already identified pH values and the activity of the enzymes that should be used for the in vitro model. As a missing input parameter for the in vitro model, we determined the residence times for three artificial feeds varying in protein $(7,12 \% \mathrm{DM})$ and carbohydrates $(8,47 \% \mathrm{DM})$ in the three midgut regions (anterior: $15 \mathrm{~min}$, middle: $45 \mathrm{~min}$, posterior: $90 \mathrm{~min}$ ). Then, we digested four artificial diets and five biowastes in the in vitro model and measured feed digestibilities (i.e. pellet DM following centrifugation) and nutrient parameters (e.g. amino acids, glucose) in the supernatant. To partially validate the simulated midgut digestion, the diets were ranked based on the supernatant nutrient results and compared to results of in vivo feeding experiments. This approach was broadly able to distinguish worst (cow manure) and best performing rearing substrates (vegetable canteen waste). But, for several diets, the performance estimated based on in vitro results did not match with the results in vivo. Future studies should try to establish a stronger correlation between in vitro and in vivo results by considering the nutrient requirements of fly larvae, hemicellulose digestion, and gut microbiota.

\section{Insects as sustainable feed for a circular economy: interdisciplinary approach to value chain development}

\author{
Y. Aartsma" and M. Dicke on behalf of the INSECTFEED consortium \\ Wageningen University and Research, Laboratory of Entomology, Droevendaalsesteeg 1, 6708 PB Wageningen, the Netherlands; \\ yavanna.aartsma@wur.nl
}

The world faces the challenge of producing food for a rapidly growing human population with an increasing demand for animal protein. Currently, livestock consumes large amounts of feed that could be used as food for humans, such as soy, using large areas of farmland and contributing to habitat and biodiversity loss worldwide. Therefore, there is an urgent need to find more efficient ways to produce feed using resources that are not suitable as food. Insects provide excellent opportunities for sustainable and circular feed production, because organic waste streams that are less suitable for livestock feed can instead be used for rearing various species of insects that can then be used for feed production. However, there are still many questions on the development of the insect sector that require investigation. These questions relate to the health and welfare of insects and insect-fed livestock, economic robustness and sustainability of the value chain and finally consumer acceptance of products derived from insect-fed animals. These issues require an integrated interdisciplinary approach. Using black soldier fly and house fly production systems, an interdisciplinary team of academic, public, private and societal partners investigates the opportunities for these species to be reared as feed for poultry. Issues that will be addressed involve how to define and measure insect welfare. They also involve the use of different substrates and their effects on insect health and welfare. Furthermore, the effects of inclusion of housefly and black soldier fly larvae in the diet of poultry will be assessed. Finally, the economic aspects and sustainability will be evaluated. With the results we aim to inform policy makers, regulators and insect producers to develop insect production systems that adhere to standards of animal welfare and health, safety and also contribute to circular economy. 


\title{
Alternative diets and their effect on the growth of Zophobas morio larvae
}

\author{
S.F.S. Cardoso ${ }^{1 *}$, T.L. Mateus ${ }^{1,2,3}$ and J.C. Lopes ${ }^{1,2}$
}

${ }^{1}$ Escola Superior Agrária, Instituto Politécnico de Viana do Castelo, Rua Escola Industrial e Comercial Nun'Álvares, 4900347 Viana do Castelo, Portugal; ${ }^{2}$ CISAS - Center for Research and Development in Agrifood Systems and Sustainability, Instituto Politécnico de Viana do Castelo, Rua Escola Industrial e Comercial Nun'Álvares, 4900-347 Viana do Castelo, Portugal; ${ }^{3}$ EpiUnit, Unidade de Investigação em Epidemiologia, Instituto de Saúde Pública da Universidade do Porto, Porto, Portugal; sarafilipacardoso@hotmail.com

Zophobas morio is a species popularly known as giant tenebrian and is commonly produced in Europe and U.S. The fact that this species is targeted for industrial production is based on its extremely high fertility, short life cycle and high feed conversion. There are still many key elements that remain little or almost unknown, such as the nutrition of the insects themselves. Thus, the goal of this study was to evaluate the influence of diet on the growth (mass $(\mathrm{mg})$ and length $(\mathrm{mm})$ ) of $Z$. morio larvae and to outline the growth curves (ADG $(\mathrm{mg} /$ day) and $\mathrm{ADC}(\mathrm{mm} /$ day)) of the respective diets. The tests were carried out at the ESA-IPVC insectarium (Portugal) under controlled temperature $\left({ }^{\circ} \mathrm{C}\right)$ and relative humidity (\%) conditions. Four diets made from conventional cereals were tested. The larvae were weighed on a precision scale $( \pm 0.0001 \mathrm{~g})$ and measured weekly, using Image $J^{\oplus}$ software, until the end of the tests, which occurred when after three successive weighing's the larvae weight remained equal. The composition and price of the diets used in this study was as follows: A - 80\% Wheat + 20\% Soy ( $€ 0.67 / \mathrm{kg}) ; \mathrm{B}-40 \%$ Barley $+40 \%$ Maize $+20 \%$ Soy $(€ 0.46 / \mathrm{kg}) ; \mathrm{C}-40 \%$ Oats $+40 \%$ Wheat $+20 \%$ Soy $(€ 0.70 / \mathrm{kg})$ and D $-40 \%$ Wheat $+40 \%$ Maize $+20 \%$ Soy $(€ 0.49 / \mathrm{kg})$. For the characterisation of the growth curve (mass and length) of the Z. morio larvae, the Gompertz model $y=A . e-e B . t-C$ ) was used, as it has the highest $\mathrm{R}^{2}$ and the best adjusts to the obtained values. Larvae fed with diet $\mathrm{B}$ had an earlier larval development time (86.3 \pm 4.0 days), whereas larvae fed with diet A had a longer larval development time (100.3 \pm 22.5 days). The larvae fed with diet A $(787.367 \pm 47.851 \mathrm{mg})$ were significantly $(P<0.01)$ less heavy than those of the other diets. The larvae fed with diet B $(56.196 \pm 6.251 \mathrm{~mm})$ obtained significantly $(P<0.05)$ shorter lengths than the larvae fed with $\operatorname{diet} C(58.000 \pm 5.320 \mathrm{~mm})$. In relation to ADG, there were significant differences $(P<0.001)$ between larvae fed with diet A $(7.943 \pm 1.203 \mathrm{mg} /$ day $)$ and those from diet B $(9.700 \pm 0.603 \mathrm{mg} /$ day $)$. Regarding the ADC and the mortality rate, there were no significant differences $(P>0.05)$ between diets.

\section{The edible caterpillar Mikombidila from the west Democratic Republic of Congo: description, life cycle and breeding}

\author{
G. Numbi Muya ${ }^{1,2}$, R. Caparros Megido ${ }^{1 *}$, F. Francis ${ }^{1}$ and B. Kambashi Mutiaka ${ }^{2}$ \\ ${ }^{1}$ Université de Liège/Gembloux Agro-Bio Tech, Passage des déportés 2, 5030 Gembloux, Belgium; ${ }^{2}$ Université de Kinshasa, \\ route de Kimwenza no. 1, Commune de Lemba, Democratic Republic of Congo; r.caparros@uliege.be
}

In the Democratic Republic of Congo (DRC), caterpillars provide up to $40 \%$ of all animal protein consumed and contributes to addressing the problems of food insecurity and poverty in the country. A great proportion of western part population of the DRC consumes caterpillars on a daily basis in their feeding habits. In this region of the country, the edible caterpillar called Mikombidila is also consumed by the inhabitants and are highly appreciated for its excellent taste. Nevertheless, this species is only available in small quantities. This species naturally feeds on a semi-recumbent annual grassy plant with ascending or erect stems up to $60 \mathrm{~cm}$ high, called Boerhavia diffusa L., belonging to the Nyctaginaceae family. Research on this caterpillar species is almost non-existent. Very few authors have described its life cycle. After rearing them under laboratory conditions, an identification of the adults has been carried out. This identification indicates that individuals belong to the Noctuidae family and Agaristinae subfamily and represents potentially the Aegocera rectilinea Boisduval 1836 species. The preliminary data on the biology of this species obtained at the end of the observation phase are as follows: it is a multivoltine species with a developmental cycle of $\sim 30$ days (egg incubation $\sim 3$ days, larval development $\sim 15$ days, pupation $\sim 12$ days) and an adult life span of $\sim 6$ days. Therefore, furthers studies are currently under process to characterise precisely its life cycle parameters and feeding preferences in order to set up a efficient and inexpensive breeding system for a rural and sustainable exploitation of the Mikombidila caterpillar in DRC. 


\title{
Alternative diets and their effect on the growth of Tenebrio molitor larvae
}

\author{
S.F.S. Cardoso ${ }^{1 *}$, T.L. Mateus ${ }^{1,2,3}$ and J.C. Lopes ${ }^{1,2}$
}

${ }^{1}$ Escola Superior Agrária, Instituto Politécnico de Viana do Castelo, Rua Escola Industrial e Comercial Nun'Álvares, 4900347 Viana do Castelo, Portugal; ${ }^{2}$ CISAS - Center for Research and Development in Agrifood Systems and Sustainability, Instituto Politécnico de Viana do Castelo, Rua Escola Industrial e Comercial Nun'Álvares, 4900-347 Viana do Castelo, Portugal; ${ }^{3}$ EpiUnit, Unidade de Investigação em Epidemiologia, Instituto de Saúde Pública da Universidade do Porto, Porto, Portugal; sarafilipacardoso@hotmail.com

Over the past five years, insects as a food source are receiving a much interest. The growing number of scientific publications and industries that have invested in their production evidences this. Despite the great advances made in the last few years on the production and use of insects, there are still many key factors that remain little or almost unknown, such as the nutrition of the insects themselves. Thus, the goal of this study was to evaluate the influence of diet on the growth (mass (mg) and length $(\mathrm{mm})$ ) of Tenebrio molitor larvae and outline the growth curves (ADG (mg/day) and ADC (mm/day)) of the respective diets. The tests were carried out at the ESA-IPVC insectarium (Portugal) under controlled temperature $\left({ }^{\circ} \mathrm{C}\right)$ and relative humidity (\%) conditions. Four diets made from conventional cereals were tested. The larvae were weighed on a precision scale $( \pm 0.0001$ g) and measured weekly, using Image ${ }^{\odot}$ software, until the end of the tests, which occurred with the appearance of the first pupa in the boxes. The composition and price of the diets used in this study was as follows: A $-80 \%$ Wheat $+20 \%$ Soy (€0.67/ $\mathrm{kg}) ; \mathrm{B}-40 \%$ Barley $+40 \%$ Maize $+20 \%$ Soy $(€ 0.46 / \mathrm{kg}) ; \mathrm{C}-40 \%$ Oats $+40 \%$ Wheat $+20 \%$ Soy $(€ 0.70 / \mathrm{kg})$ and D $-40 \% \mathrm{Wheat}$ $+40 \%$ Maize $+20 \%$ Soy $(€ 0.49 / \mathrm{kg})$. For the characterisation of the growth curve of the T. molitor larvae, the exponential equation mass or length $=e(B 0+B 1 . t)$ was used, as it is the one with the highest $\mathrm{R}^{2}$ and which best fits the values obtained. $T$. molitor larvae, fed with diet $\mathrm{B}$, obtained significantly the highest final mass $(143.444 \pm 10.349 \mathrm{mg})(P<0.01)$ and longest length $(25.207 \pm 2.232 \mathrm{~mm})(P<0.001)$. Diet B was also the one in which the larvae reached the pupal phase earlier $(23.3 \pm 4.04$ days $)$, while the larvae fed with diet A were the latest ( $40 \pm 8.08$ days). There were no significant differences in the mortality rate between diets. In relation to ADG, it was found that there were significant $(P<0.05)$ differences between larvae fed with diet A $(2.340 \pm 0.858 \mathrm{mg} /$ day $)$ and larvae fed with diet B $(4.452 \pm 0.959 \mathrm{mg} /$ day $)$. With regard to the ADC, the larvae fed with diet B $(0.708 \pm 0.081 \mathrm{~mm} /$ day $)(P<0.01)$ obtained average daily growths higher than those of the other diets. Diet B showed lower production cost and less time for larval involvement when compared to the traditional wheat bran diet. 


\title{
Insect production systems/processing organic side streams \& waste management
}

\section{Impact of different gut bacteria on soybean curd residue co-conversion by black soldier fly larvae}

\author{
A.A. Soomro ${ }^{1}$, M. Cai ${ }^{1}$, K. ur Rehman ${ }^{1,2}$, L. Zheng ${ }^{1}$, X. Xiao ${ }^{1}$, A. Mathys ${ }^{3}$, M. Gold ${ }^{3,2}$, J.K. Tomberlin ${ }^{5}$, Z. Yu ${ }^{1}$ and \\ J. Zhang ${ }^{*}$ \\ ${ }^{1}$ State Key Laboratory of Agricultural Microbiology, National Engineering Research Center of Microbial Pesticides, College \\ of Life Science and Technology, Huazhong Agricultural University, Wuhan, 430070, China, P.R.; ${ }^{2}$ Livestock and Dairy \\ Development Dept., Poultry Research Institute, Government of Punjab, Pakistan; ${ }^{3}$ Laboratory of Sustainable Food Processing, \\ Institute of Food, Nutrition and Health, ETH Zurich, Zürich, Switzerland; ${ }^{4}$ Dept. of Sanitation, Water and Solid Waste \\ for Development (Sandec), Swiss Federal Institute of Aquatic Science and Technology (Eawag), Dübendorf, Switzerland; \\ ${ }^{5}$ Dept. of Entomology, Texas AEM University, USA; zhangjb@mail.hzau.edu.cn
}

The processing of organic waste with black soldier fly larvae is an emerging waste treatment technology. To enhance the conversion efficiency of soybean curd residues (SCR) by black soldier fly larvae (BSFL) assisted by different gut bacteria and subsequently obtain high-quality insect protein and lipid for human and livestock consumption, we pretreated SCR with different gut bacteria $\left(10^{8} \mathrm{cfu} / \mathrm{ml}\right)$ and used BSFL for conversion. All gut bacterial strains affected BSFL conversion efficacy, nutrition, and safety. FCR and protein and lipid content in BSFL are the most important parameters for evaluating BSFL conversion efficacy. Among the bacterial strains, BSFL with Bacillus subtilis BSFL-CL (Group R1) showed the highest co-conversion efficiency and best performance and showed significant enhancement in nutrient content and decrease in FCR. BSFL assisted by microbes enhanced the co-conversion process of BSFL and reduced the development time of BSFL from 19 days to 15 days. Furthermore, BSFL assisted with B. subtilis BSF-CL (Group R1) had significantly higher dry mass reduction (58.0\%) and bioconversion (6.4\%) vs control. The nutritional profile in BSFL protein (50.5\%) and lipid (28.2\%) were enhanced in Group R1, and the FCR (8.9) of BSFL fed with SCR and B. subtilis BSF-CL (Group R1) was significantly lower than that of BSFL fed with SCR different other gut bacteria. Therefore, for further nutritional investigations we chose group R1. In addition, amino acids and fatty acid spectra developed on group R1 showed a satisfactory and higher level of all the essential amino acids needed for human consumption when compared with WHO/FAO standards. All heavy metals and antinutritional factors were also found within the safe intake level of human animal food production. In conclusion, when B. subtilis BSF-CL was assisted with BSFL developed on SCR improved all its performance parameters while development time and FCR significantly decreased. Furthermore, in nutritional values such as, protein and lipids contents in BSFL also increased as compared to control groups. 


\title{
Physical characterisation of black soldier fly rearing diets
}

\author{
L. Frooninckx", S. Goossens, A. Wuyts and S. Van Miert \\ Thomas More University College of Applied Sciences, RADIUS, Kleinhoefstraat 4, 2440 Geel, Belgium; \\ lotte.frooninckx@thomasmore.be
}

Larvae of the black soldier fly (BSF), Hermetia illucens, are voracious feeders that can grow on a wide variety of organic materials. When grown on organic side streams, they are able to convert such low value materials into high value biomass composed of fats, proteins and chitin. These components can be used as a sustainable resource for food, feed and technical applications. The potential of a side stream as an ingredient of a rearing diet for BSF depends among other things on its chemical and physical properties. The influence of chemical parameters such as $\mathrm{pH}$, macronutrient concentration and ratio have already been reported in literature, but the influence of the physical characteristics have been neglected so far. In this study we investigate the influence of dynamic and kinematic viscosity, syneresis and water holding capacity of semi-artificial BSF rearing diets on growth, biomass conversion and material reduction of BSF larvae. The output of these experiments will give us more insight on the potential of side streams with specific chemical and physical properties as an ingredient of a BSF rearing diet.

\section{Does juice make them fat - processing grass juice with black soldier fly larvae (Hermetia illucens)}

\author{
C.L. Coudron", D. Deruytter, J. Claeys and S. Teerlinck \\ Inagro, Ieperseweg 87, 8800 Rumbeke-Beitem, Belgium; carl.coudron@inagro.be
}

Grass clippings from maintenance of our roadside verges pose a problem throughout Europe. The high volumes of biomass involved, result in high (waste) processing costs. Both industry and local authorities, however, are interested in the possibility of using roadside grass clippings as a novel biobased resource. One possible application is using the grass fibres for the production of building panels and bio-composites. This requires separating the solid (fibres) and the liquid fractions. The liquid fraction (grass juice) contains the grass proteins. The feasibility of bio-converting these proteins to insect protein was assessed by using the juice as a feedstock for black soldier fly larvae. The larvae of the black soldier fly prefer a moist (but not wet) rearing substrate (containing 30\% dry matter). Grass juice is a liquid (7\% DM), meaning it cannot be fed in its pure, unprocessed form. An attempt was made to increase the dry matter content of the juice by evaporating a part of the moisture (going from 7 to $30 \%$ ). However the grass syrup afterwards was an extremely unfavourable environment for the larvae resulting in high mortality and slow growth, forcing us to abandon this idea. In the second trial, the raw grass juice was fed in combination with a dry feedstock. Three different dry feeds were assessed with varying nutritional compositions: (1) spelt husks as a low nutritious feedstock; (2) wheat bran as an intermediate feedstock; and (3) chicken feed as a nutrient-rich feedstock. These three diets were compared with three diets where the grass juice was replaced with water (all in triplicate). Growth was assessed by comparing total fresh and dry larval yield. Feed efficiency (as dry feed/dry harvest) and nitrogen retention efficiency (as a percentage of $\mathrm{N}$ retained/ $\mathrm{N}$ fed) were determined for all diets. Results showed black soldier fly larvae are an efficient way to dry and process grass juice if it is properly combined with a dry feedstock. Especially if the dry feedstock is of low nutritional quality (spelt husks), grass juice will improve growth compared to water (a nitrogen retention of $38.5 \pm 3.4 \%$ (SD) compared to $28.9 \pm 0.6 \%$ (SD) respectively). For nutritious feedstocks such as chicken feed, feed efficiency and larval yield did not differ significantly for grass juice and water (a nitrogen retention of $61.6 \pm 0.7 \%$ (SD) compared to $58.0 \pm 1.9 \%$ (SD) respectively), but more important, no adverse effects could be observed despite the fact that $10 \%$ less dry feedstock was used in the diet. To place these numbers in perspective, broiler chickens have a similar nitrogen retention as black soldier fly on chicken feed. 


\title{
Maturity and fertilising potential of black soldier fly manure (frass) fed residual organic matter from grocery stores
}

\author{
L. Hénault-Ethier ${ }^{1,2,3^{*}}$, M. Kone ${ }^{1}$, M.H. Deschamps ${ }^{1}$ and G.W. Vandenberg ${ }^{1}$ \\ ${ }^{1}$ Faculté des Sciences de l'Agriculture et de l'Alimentation, Université Laval, Québec (QC) Canada, ${ }^{2}$ TriCycle Inc, Montreal \\ (QC) Canada; ${ }^{3}$ David Suzuki Foundation, Montréal (QC) Canada; louise@tricycle.eco
}

The mass production of black soldier fly larvae (BSF) is an opportunity to bioconvert many types of residual organic matter (ROM) in sustainable alternative livestock feed ingredients rich in proteins and lipids. By products of this upcycling - called frass - include faeces, exuviae rich in chitin and residual organic matter and may offer interesting fertilising potential. This study examined how BSF larvae convert pre-consumer organic matter collected from grocery stores in the Quebec City area. ROM was composed of $52 \%$ vegetables, $38 \%$ fruits and $10 \%$ bread and cereals products. Six different iso-lipidic (3.6\% dw) and iso-energetic (18.3 MJ $/ \mathrm{kg}$ ) containing $85 \%$ moisture (plus a $70 \% \mathrm{RH}$ reference) were formulated with varying ratios of ROM $(0$, $30,50,70$ and $100 \%$ ) incorporated to the Gainesville control (50\% wheat bran, $30 \%$ alfalfa and $20 \%$ corn, mass in dry weight). A total of $144 \mathrm{~g} \mathrm{dw}$ were given to 600 larvae per bin, and the experiment was conducted in triplicate. Frass was collected following 10-13 days growth experiments conducted at $27^{\circ} \mathrm{C}$ and $80 \%$ relative humidity. Higher moisture content prevented microbial decomposition activity based on temperature remaining at ambient levels and low pH $(<5)$. Despite a stable $\mathrm{C} / \mathrm{N}$ ratio, enhanced mineralisation of the Gainesville diets increased from day 10 to 13 while nitrogen availability $\left(\mathrm{N}^{-N_{4}} / \mathrm{N}_{\text {tot }}\right)$ dropped in higher moisture treatments. Because the $\mathrm{C} / \mathrm{N}$ ratio and moisture content of the frass for all treatments outside the optimal range for composting (25-30/1 and $>65 \%$, respectively) addition of a dry carbon rich bulking agent is recommended for further biological stabilisation through composting. The fertilising value (N-P-K) of $13 \mathrm{~d}$ old frass harvested from the Gainesville 70\% RH treatment is 6-9-8, but only 3-3-3 in the control at 85\% RH and 3-1-4 in the 100\% ROM treatment. All Gainesville controls contained higher concentrations of trace elements (Ca, Mg, Mn, $\mathrm{Zn}, \mathrm{Fe}$ ) and OM compared to treatments with increasing concentrations of ROM, except for $\mathrm{Na}$ which increased with the addition of ROM $(\leq 0.05 \%)$, while remaining at least 100 times below maximal agronomic guidelines (5\%). In conclusion, BSF frass appears to have some interesting soil conditioning $(10-22 \% \mathrm{OM})$ and fertilising properties, but based on laboratory scale results obtained, it may require additional stabilisation through composting before it can safely be used as a soil amendment.

\section{Maximising mycotoxin bioremediation of agriculture wastes: mealworms and dried distillers grains with solubles}

\author{
H.C. Kelstrup ${ }^{1 *}$, D. Tweedy ${ }^{1,2}$, J. Morales-Ramos ${ }^{2}$, G. Rojas ${ }^{2}$ and V.J. Emery ${ }^{1}$ \\ ${ }^{1}$ Beta Hatch Inc., Cashmere, Washington, 200 Titchenal Road, Cashmere, WA 98815, USA; ${ }^{2}$ USDA-ARS National Biological \\ Control Laboratory, 59 Lee Road, Stoneville, MS 38774, USA; hans@betahatch.com
}

Insects show great potential as a natural tool for the bioremediation of mycotoxin-contaminated feed wastes. Our research along with those of others have shown that the yellow mealworm (Tenebrio molitor) can dramatically reduce the level of mycotoxins (e.g. aflatoxin, deoxynivalenol and zearalenone) in feed, and the concentration of the toxin does not appear to impact larval growth or mortality. The detoxifying prowess of the mealworm gut may open new waste streams that insect growers can uniquely exploit. For example, ethanol production from corn fermentation and distillation creates a by-product called dried distillers grains with solubles (DDGS). Corn is one of the most susceptible crops to mycotoxin contamination, and mycotoxins are concentrated $3 \times$ in the DDGS, making it problematic to sell as conventional animal feed. Yet for insects, contaminated DDGS pose no such problem, and our research is showing that DDGS is a preferred ingredient for the yellow mealworm. However, due to their high protein and lipid but low carbohydrate content, DDGS are not adequate as insect diets when used in high concentrations or by themselves. In this presentation, we report results from our research on mycotoxin detoxification by the yellow mealworm, and in turn show the progress we have made in formulating diets high in corn DDGS that will maximise mycotoxin bioremediation, while at the same time providing adequate nutrition for mealworm production. 


\title{
Mite infestation and its risk assessment to a black soldier fly colony: identification, management and solutions
}

\author{
T. Bruder \\ Nasekomo EAD, Business Park Sofia, 1166 Sofia, Bulgaria; thiphaine.bruder@nasekomo.life
}

Keeping a colony healthy is a key challenge for insect mass production in industrial rearing facilities. A growing number of scientific papers investigate the ability of insects like the black soldier fly (BSF; Hermetia illucens) to convert organic wastes into high quality nutrients for feed and food. This bioconversion often involves survival in a wide variety of feed ingredients including ones with high contaminations in heavy metals, pesticides, viruses, bacteria, etc. At the same time, ideal environmental conditions (warm temperatures and high air humidity) to rear the BSF may attract unwanted animals: other species of insects such as the housefly (Musca domestica) or the fruit fly (Drosophila melanogaster), but also, nematodes, mites, etc. These pests can compete on the feed source through rapid colonisation thanks to short lifecycles and fast consumption of the substrate. They also introduce sanitary risks as they may carry pathogens which present a real danger to the entire colony but also for the staff rearing insects in industrial settings. An important knowledge gap remains on these potential pests, pathogens and parasites of the egg, larvae, pupae and adult stages of the BSF. This presentation shares our experience with a mite invasion our BSF colony suffered during summer 2019. Mites are tiny arthropods, barely visible without magnification, which belong to the class Arachnida and subclass Acari. They are potent threats to honeybees and to other wild fly colonies with Varroa destructor species as a well-known example. Harmless mites are also frequently found in cereal by-products stocks, common ingredients used in insect feed. We will detail the different steps that allowed us to identify mites in our facility, determine their specie and their provenance. After discovering a mite population in our BSF larvae growing boxes and on our adult flies, we evaluated their harmful nature and contacted researchers specialised in arthropods to identify and understand our aggressor. Furthermore, we investigated to what extent every BSF life stage was impacted by the mites. We performed on quarantined boxes and cages different experiments with potential inhibiting strategies such as oxalic acid exposure, zeolite spreading, feed heat treatments and frass sorting. We describe how we evaluated operational impacts and associated risks for our colony and how to control them up to complete eradication of the pest without stopping production. This is an opportunity to discuss our approach to this crisis, to give some guidance on operations and bio-security best practices and to share preventive measures. The need for more research and collaborations on this strategic matter for the future of insect farming is also hereby highlighted.

\section{Black soldier fly larvae rearing on side streams: impact on bacterial biota in different gut regions and larval growth}

\author{
D. Vandeweyer ${ }^{1 *}$, D. Bruno ${ }^{2}$, J. De Smet ${ }^{1}$, M. Bonelli ${ }^{3}$, M. Casartelli ${ }^{3}$, G. Tettamanti ${ }^{2}$ and L. Van Campenhout $^{1}$ \\ ${ }^{1}$ KU Leuven, Department of Microbial and Molecular Systems, Lab4Food, Campus Geel, Kleinhoefstraat 4, 2440 Geel, \\ Belgium; ${ }^{2}$ University of Insubria, Department of Biotechnology and Life Sciences, via J.H. Dunant 3, 21100 Varese, Italy; \\ ${ }^{3}$ University of Milano, Department of Biosciences, via Celoria 26, 20133 Milano, Italy; dries.vandeweyer@kuleuven.be
}

Black soldier fly (Hermetia illucens) larvae (BSFL) are capable of digesting a broad range of substrates and to convert them into protein-rich biomass. Differences in the nutritional composition of feeding substrates may result in differences in gut microbial profiles in the larvae, during the rearing cycle as well as at the end of the whole cycle. Moreover, depending on the insect developmental stage and the region of the gut, differences in microbial profiles may occur. General insight in these aspects may help to optimise the growth of larvae. When BSFL are reared to serve as ingredient in animal feeds, the highest added value can be generated by using low-quality (industrial) side streams as substrates, such as dried distillers grains with solubles (DDGS) and organic kitchen waste (swill). Therefore, the aim of this study was to rear BSFL on DDGS and swill, and monitor their microbiota in function of larval development and gut regions. Moreover, the growth rate of the larvae was compared with their performance on commercial chicken feed as control. During and after rearing, samples of the substrate, larvae, and residue were taken to monitor their bacterial biota. Four different gut regions were isolated from last instar larvae: the anterior, middle and posterior midgut, and the hindgut. The bacterial composition in the different gut districts for the three substrates are now being determined to study the community in each region and their similarity with the whole larvae. Also the bacteria in the substrates and in the residues will be determined to identify correlations. Illumina MiSeq sequencing targeting the 16S rRNA genes within the total DNA from the sample is currently being performed. This technology only provides relative abundances of bacterial genera present in the samples. Therefore, in addition quantitative real-time PCR (qPCR) is being performed, as this allows to transform relative into absolute abundances, giving a more realistic view on the bacterial community. As to growth performance, the weight gain of the BSFL was very comparable when reared on DDGS or chicken feed. After 14 days of rearing, the larvae reached a maximum weight of approximately $300 \mathrm{mg}$. Larvae reared on swill clearly grew slower and their weight did not exceed $200 \mathrm{mg}$, even after 21 days of rearing. 


\title{
Influence of temperature on selected life-history traits of black soldier fly (Hermetia illucens) reared on two common urban organic waste streams in Kenya
}

\author{
M. Shumo ${ }^{1,2 *}$, F.M. Khamis ${ }^{2}$, C.M. Tanga ${ }^{2}$, K.K.M. Fiaboe ${ }^{2,3}$, S. Subramanian ${ }^{2}$, S. Ekesi ${ }^{2}$, A. van Huis ${ }^{4}$ and \\ C. Borgemeister ${ }^{1}$ \\ ${ }^{1}$ Center for Development Research (ZEF), Dept of Ecology and Natural Resources Management, 53113 Bonn, Germany; \\ ${ }^{2}$ International Centre of Insect Physiology and Ecology (icipe), Plant Health Unit, Nairobi 00100, Kenya; ${ }^{3}$ IPM Department, \\ The International Institute of Tropical Agriculture (IITA), B.P. 2008 (Messa), Nkolbisson, Yaoundé, Cameroon; ${ }^{4}$ Dept of \\ Plant Sciences, Laboratory of Entomology, Wageningen University \& Research, 6700 AA Wageningen, the Netherlands; \\ mshummo@uni-bonn.de
}

In sub-Saharan Africa, urban populations are projected to increase by $115 \%$ in the coming 15 years putting high pressure and demand on agricultural production. The high ecological footprint of meat and dairy production, prevents the livestock sector from meeting the increasing demand in a sustainable manner. Insects such as the black soldier fly (BSF) are rich in nutrients and can be reared on organic side streams. Substrates derived from organic byproducts are suitable for industrial large-scale production of insect meal. Although efficient in waste management and in feed production, BSF larvae are very sensitive to the external environment such as temperature and rearing medium. Therefore, we studied the effect of temperature and substrate type, i.e. brewers' spent grain (SG) and cow dung (CD), on the development and survival of BSF larvae. Both organic substrates were readily available in Nairobi, Kenya, the location of the experiments. In our experiment, 100 3-5-day-old BSF larvae were fed either SG or CD and further treated at temperatures of $15,20,25,30$ and $35^{\circ} \mathrm{C}$. The duration of larval development was recorded, and the prepupae were removed, weighed, and placed individually in separate, labelled, 35-ml plastic cups filled with moist sawdust. After emergence, 102 -day-old adults ( 5 males and 5 females) from every replica per substrate were transferred into a cage $(40 \times 40 \times 40 \mathrm{~cm})$ and allowed to mate for $24 \mathrm{~h}$ at their respective temperatures. The laid egg batches were collected and counted, and the adult flies' longevity was recorded. The data were subjected to a two-way analysis of variance (ANOVA) using the general linear model procedure. BSF larvae reared on SG developed faster than those reared on $\mathrm{CD}$; the former also favoured higher temperatures for their larval development and emergence into adults. The optimum range was $25-30{ }^{\circ} \mathrm{C}$. With increasing temperatures, the longevity of adult BSF decreased, while the fecundity of females increased. Thus, it is possible to take advantage of the readily available SG waste streams in the urban environments of Kenya to produce BSF larvae-derived livestock feed within a short duration of time and at relatively high temperatures.

\section{Effect of including chicken manure with pre-consumer waste on black soldier fly larval performance and bioconversion}

\author{
N.J. Parry ${ }^{1 *}$ and C.W. Weldon \\ Department of Zoology and Entomology, University of Pretoria, Pretoria, South Africa; nina.jparry@gmail.com
}

The black soldier fly (BSF), Hermetia illucens L. (Diptera: Stratiomyidae), is commonly used in bioconversion due to its versatility and efficiency in reducing various types of organic waste, including fruit and vegetable waste and animal manure. The type of waste that larvae feed on can impact their development time, performance and total biomass. Most studies have investigated only one type of waste at a time, but varying mixtures can lead to improved larval performance and bioconversion. This study investigated the effects of mixtures of chicken manure with fruit and vegetable pre-consumer waste on BSF larval development and bioconversion. Chicken manure was mixed with pre-consumer waste in amounts varying from 0 to $100 \%$. Freshly hatched BSF neonates $(90 \mathrm{mg})$ collected within three hours of hatching, were placed on $12 \mathrm{~kg}$ of each mixture. The larvae were held at $28 \pm 0.5^{\circ} \mathrm{C}$ and this was repeated for fifteen different batches of each chicken manure and pre-consumer waste mix. The development rate, final total biomass, final individual mass, total waste reduction, bioconversion and efficiency of conversion of digested feed (ECD) were recorded and compared between the tested groups using general additive models in R. As the proportion of chicken manure increased in the mixtures, individual larvae reduced in size while total development time increased. Bioconversion efficiency and total biomass produced on a dry matter basis decreased dramatically when chicken manure was greater than $40 \%$. However, total chicken manure in the mixture did not impact survival of the larvae or waste reduction. Initial temperature and $\mathrm{pH}$ of the mixture during seeding also had a significant effect on larval development, survival, biomass production and waste reduction. Mixes of fruit and vegetable waste can include up to $40 \%$ chicken manure without compromising survival, waste reduction, larval development or biomass production. 


\title{
The influence of residue microbes on black soldier fly larvae (Hermetia illucens) treatment of two agricultural byproducts
}

\author{
M. Gold ${ }^{1,2 *}$, T. Fowles ${ }^{3}$, J.D. Fernandez-Bayo ${ }^{4}$, L. Palma ${ }^{4}$, C. Zurbrügg ${ }^{2}$, C. Nansen ${ }^{3}$, H. Bischel ${ }^{5}$ and A. Mathys ${ }^{1}$ \\ ${ }^{1}$ ETH Zurich, Sustainable Food Processing, Schmelzbergstrasse 9, 8092 Zurich, Switzerland; ${ }^{2}$ Eawag, Department of \\ Sanitation, Water and Solid Waste for Development (Sandec), Überlandstrasse 133, 8600 Dübendorf, Switzerland; ${ }^{3} U C$ \\ Davis, Department of Entomology and Nematology, Briggs Hall, Davis, CA 95616, USA; ${ }^{4}$ UC Davis, Dept of Biological and \\ Agricultural Engineering, Bainer Hall, Davis, CA 95616, USA; ${ }^{5}$ UC Davis, Dept of Civil and Environmental Engineering, \\ Ghausi Hall, Davis, CA 95616, USA; moritz.gold@hest.ethz.ch
}

Black soldier fly larvae (BSFL) are known to dramatically alter the microbial community of their substrates, yielding microbialrich residues. Recently, studies found that the addition of pure-culture bacteria and/or defined bacteria mixtures to substrates can increase BSFL bioconversion efficiency. However, maintaining microbial communities in pure-culture alongside BSFL production is not always practical. We hypothesised that recycling of the residue into the substrate, or inoculating the native substrate microbial community with concentrated, residue-derived microorganisms, would enhance BSFL bioconversion efficiency. BSFL were reared on two agricultural byproducts (tomato and white wine pomace) with replicate reactor setups (i.e. open and closed reactors: $3-4 \times 200$ larvae, $1 \mathrm{mg}$ DM/larva). Treatment reactor setups consisted of the same agricultural by-product with variable amounts (20-60\% DM) of the substrate replaced by residue. Alternatively, the substrate was spiked with microbial inoculants derived from the residue (non-selective nutrient broth, $30^{\circ} \mathrm{C}, 24 \mathrm{~h}$ ). Substrate nutrient content, substrate and residue aerobic viable counts, $\mathrm{pH}$ and temperature (every $10 \mathrm{~min}$ ) were determined. Pre-consumer food waste served as a high-performance reference. The addition of the residue or residue-derived microbial communities decreased the BSFL bioconversion efficiency for tomato pomace ( $49 \pm 1.5 \mathrm{mg} \mathrm{DM} /$ larva for treatment vs $44 \pm 1.8 \mathrm{mg} \mathrm{DM} / \mathrm{larva}$ for control) and had no effect on the bioconversion of white wine pomace $(20 \pm 2.1 \mathrm{mg}$ DM/larva for treatment vs $21 \pm 1.4 \mathrm{mg}$ DM/larva for control). The maximum temperature of the substrate/residue during the experiment was higher in treatments with the inoculants, and $\mathrm{pH}$ and microbial numbers similar to the control. Larval gut and residue microbial community composition is being determined with DNA-based sequencing. The process performance between substrates increased with nutrient content and was highest for pre-consumer food waste (e.g. $50 \mathrm{mg} \mathrm{DM} / \mathrm{larva}$ ) and lowest for white wine pomace (e.g. $20 \mathrm{mg}$ DM/larva). Higher performance gains in insect production systems can be achieved via balanced and/or high nutrients substrates rather than by incorporating microbial inoculants.

\section{Introduction to the EU H2020 project SUSINCHAIN (SUStainable INsect CHAIN)}

\author{
T. Veldkamp ${ }^{1 *}$, H.J. van der Fels-Klerx ${ }^{2}$, N.P. Meijer ${ }^{2}$ and M. Octavia ${ }^{3}$ \\ ${ }^{1}$ Wageningen Livestock Research, P.O. Box 338, 6700 AH Wageningen, the Netherlands; ${ }^{2}$ Wageningen Food Safety Research, \\ Akkermaalsbos 2, 6708 WB Wageningen, the Netherlands; ${ }^{3}$ Sociedade Portuguesa de Inovacao, Av Marechal Gomes da \\ Costa 1376 POR, 4150356 Porto, Portugal; teun.veldkamp@wur.nl
}

Insects and insect-derived products have been in the European market since 2010, however scaling up the insect value chain in Europe is progressing at a slow pace. The SUSINCHAIN project is to overcome the barriers for increasing economic viability of the insect value chain and opening markets. This will be done by combining forces in a comprehensive multiactor consortium with 18 industry partners and 17 academic partners. SUSINCHAIN considers the entire insect value chain from farm (insect rearing) to fork (insect consumption) based on black soldier fly, housefly, mealworm and crickets. The SUSINCHAIN concept is based on performing all activities (innovations, testing, demonstrating, sharing knowledge) to overcome key important hurdles for scaling up the European insect value chain. Practical experience of industry partners addressing supply-side barriers as well as related risk management strategies will be evaluated and best practices elaborated.. Insect rearing innovations will be focused on organic side streams of vegetable origin not used as animal feed. For improving economic viability, possibilities for marketing of rearing by-products will be evaluated and strategies to avoid insect diseases will be developed. Transport, storage and processing technologies for insects will be optimised at large scale. Microwave and radio frequency drying, high moisture extrusion and protein recovery from fresh larvae by using enzymatic pre-treatment combined with continuous tricanter centrifugation will be validated and demonstrated. Controlled atmosphere packaging and preservation for storage and transport of living insects will be tested. The digestibility of insect meals produced by different processing methods and included in feed formulae for large-scale level commercial diets to test optimal inclusion levels to maintain or increase livestock performance and health. Insect based food products will be designed and developed suitable for the domestic preparation of regular dinner meals. The microbiological, chemical and allergenic safety of insects and derived products will be addressed along the entire chain. Hygiene codes, HACCP protocols, and guidelines for safe insect production will be created and distributed among the supply chain actors. Furthermore, a decision support system will be developed to ensure the sustainable growth of the insect value chain in Europe, in addition to the stakeholder's platform provided within the context of the project in order to connect and receive valuable inputs from the relevant actors. 


\title{
Degradation and proposed pathway of ciprofloxacin by black soldier fly larvae combined with its intestinal microbes
}

\author{
C. Yang ${ }^{1}$, S. Ma ${ }^{1}$, M. Cai ${ }^{1}$, L. Zheng ${ }^{1}$, J.K. Tomberlin ${ }^{2}$, Z. Yu ${ }^{1}$ and J. Zhang ${ }^{1^{*}}$ \\ ${ }^{1}$ Key Laboratory of Agricultural Microbiology, National Engineering Research Centre of Microbial Pesticides, College of \\ Life Science and Technology, Huazhong Agricultural University, Wuhan, 430070, China, P.R.; ${ }^{2}$ Department of Entomology, \\ Texas AEM University, USA; zhangjb@mail.hzau.edu.cn
}

Antibiotics are widely used in livestock and poultry farming because of their unique ability to inhibit and kill pathogens, but it is difficult for animals to degrade them. Most antibiotics will be excreted with livestock and poultry. Excretion of manure containing antibiotics into the environment without treatment can pose a significant threat to ecosystems and public health. Although traditional compost can remove some antibiotics, it takes a long time and is enriched with a large number of antibioticresistant bacteria and resistance genes. In this paper, we studied the degradation effect of ciprofloxacin (CIP) by black soldier fly larvae (BSFL) in artificial feed. The results showed that the degradation rate of CIP was detected to be $85.48 \%$ artificial feed, which was four folds that of the control. Then the BSFL intestinal microbe role in CIP degradation was explored. Five ciprofloxacin-resistant bacteria were isolated from the gut of the BSFL, and identified by morphology and molecular biology as BSFLG-CIP1 (Klebsiella Pneumoniae), BSFLG-CIP2 (Trichosporon asahii), BSFLG-CIP3 (Geotrichum sp.), BSFLG-CIP4 (Pichia kudriavzevii) and BSFLG-CIP5 (Proteus mirabilis), in which BSFG-CIP1 and BSFLG-CIP5 have degradation rate of CIP of 98.22 and $97.83 \%$, respectively. However, their growth has also been affected by the concentration of CIP. In the process of using the sterile BSFL system to research the degradation of ciprofloxacin by BSFL and intestinal microbes, the degradation rate of CIP by sterile BSFL was $21.76 \%$. After the addition of intestinal microbes, the degradation rate of CIP obviously improved indicated that the gut microbes play an important role in the degradation process of ciprofloxacin. Seven kinds of possible metabolites were identified by in vitro degradation of CIP by BSFL intestinal microbes, and their metabolic pathways were preliminarily inferred.

\section{Dynamics of the occurrence of the ARGs during cattle manure processing by Hermetia illucens}

\author{
J. Vašíčková", D. Zimčík, L. Micenková and T. Persaň \\ Masaryk University, Faculty of Science, RECETOX, Kamenice 753/5, 62500 Brno, Czech Republic; \\ jana.vasickova@recetox.muni.cz
}

Multiple veterinary pharmaceuticals, including veterinary antibiotics (VAs), are extensively applied worldwide as growth promoters and therapeutic agents in livestock production. The widespread use and continuous release of VAs into the environment accelerates the development of antibiotic-resistant microbial populations and facilitates the maintenance of the resistance at high levels. Several studies explored that manure is a reservoir of antimicrobial-resistant bacteria and even when livestock is not administered with antibiotics, a background level of resistance exists in faeces, which can fluctuate based on feed ingredients in animal diets. Compared to conventional composting, black soldier fly (BSF) larvae Hermetia illucens offer a promising alternative for the biodegradation of animal manure. As an ecological decomposer, BSF larvae are often exposed to relatively high concentrations of harmful microorganisms, such a bacteria and fungi. Recently, many reports have identified that the presence of specific microflora in the insect gut play an important role in the host immune system, which consequently prevents colonisation of pathogens, and hence is involved in maintaining host health. Thus, the potential inactivation of pathogens by the BSF larvae microbiome complex could be useful in the manure sanitisation. The ability and mechanisms of BSF larvae associated intestinal microbes to degrade ARGs were investigated in this study. The goal of the presented project was to study the attenuation of selected ARGs during BSF larvae-composting and conventional composting. The relative abundances of nine ARGs conferring sulphonamides resistance (sul1, sul2 and sul3) and MLSB resistance (ermA, ermB, ermC, ermF, ermT, and ermX) were determined with established methods of real-time PCR. The result of this study contributes to understanding the effects of BSF larvae on the occurrence of ARGs in cattle manure. The understanding of how BSF larvae influence the degradation and presence of ARGs during manure processing can significantly help to mitigate their spread in the environment. This project was supported by GJ19-11782Y. 


\title{
The role of the black soldier fly, Hermetia illucens in combating municipal waste in Windsor-Essex
}

\author{
P.O. Okpara and S.L. VanLaerhoven" \\ University of Windsor, 401 Sunset Avenue, N9B 3P4, Windsor, ON, Canada; vanlaerh@uwindsor.ca
}

Within Ontario alone, approximately 3.7 million tonnes of organic food waste are generated yearly. This waste includes food scraps, wasted food and greenhouse wastes such as leaves, plant stems, and fruits. More than $55 \%$ of this waste is generated by the residential and municipal sectors and most of it is transported to landfills for composting each year. The use of landfills as a method of waste management is not sustainable. It adds strain to the environment by releasing harmful greenhouse gases and demands landfill space. It is projected that based on the current trends in economic growth, more than 16 landfills will be required by 2050 in Ontario if more progress is not made to reduce the use of landfills for organic waste disposal. Currently, the protocols set by the Ontario government include educational tools to support waste prevention strategies, safety guidelines to support the safe donation of excess food, and province ban of organic waste sent to disposal sites. While all these are strong protocols, there is a need to address methods that support recovery and converting food waste into valuable end products that could contribute to economic benefits (i.e. circular economy). The black soldier fly, Hermetia illucens Linnaeus (Diptera: Stratiomyidae) has been shown to reduce a range of waste, from all-vegetable to all-meat. The goal of this research was to examine the potential for $H$. illucens as a sustainable waste management method for municipal waste in the Windsor-Essex region by evaluating life-history traits, waste reduction efficiency and waste reduction index of larvae reared on municipal waste and chicken feed as a control. Treatments consisted of 150 4-day-old larvae fed either municipal waste or chicken feed every 2 days. When comparing across treatments larvae developed faster on chicken feed $(P<0.05)$ and showed a higher waste reduction index $(P<0.05)$. However, waste reduction efficiency was the same across treatments $(P>0.05)$ and prepupae reared on municipal waste weighed more than those reared chicken feed $(P<0.05)$. Data from this study shows that $H$. illucens can be a suitable and sustainable waste management alternative. There were physiological trade-offs observed as a result of larval feeding on a heterogenous diet such as municipal waste.

\section{Chemical composition of Musca domestica maggots fed different agricultural substrates from ther region Niamey, Niger}

\author{
I. Hamidou Leyo ${ }^{1}$, Z. Moussa Ousman ${ }^{1}$, F. Francis ${ }^{2}$ and R. Caparros Megido ${ }^{2 *}$ \\ ${ }^{1}$ University of Abdou Moumouni de Niamey, Faculty of Agronomy, College life and Earth Science (EDSVT), BP 10 960, \\ Niamey, Niger; ${ }^{2}$ University of Liège, Faculté Gembloux Agro-Bio Tech, Unité Entomologie fonctionnelle et évolutive, Passage \\ des Déportes 2, 5030 Gembloux, Belgium; r.caparros@uliege.be
}

As the need for high-quality and innovative chicken feed in Niger is increasing rapidly, the objective of this work was to investigate the potential of maggot production on locally available byproducts as a novel feed for poultry. Eight substrates (cow manure; cow rumen content; sorghum stem residue; millet glume; rice husk; brewer's grains; millet bran and wheat bran) were selected due to their open and easy access in the agricultural environment of Niamey. To test these monodiets, Musca domestica eggs were obtained from a $M$. domestica breeding unit set up at the university of Abdou Moumouni. Each diet $(\mathrm{n}=3)$ was inoculated with $0.01 \mathrm{~g}$ of $M$. domestica eggs. On day 5 , the larval biomass of each substrate was collected and weighed per replica as well as the weight of 20 maggots per substrate per replica. After extraction of the larvae, $30 \mathrm{~g}$ of final residue per replica was collected for biochemical analysis. Preliminary results showed that the fresh larval biomass is influenced by the type of substrate used. The maximum and minimum fresh biomass were obtained with millet bran $(3.638 \pm 0.334 \mathrm{~g})$ and ruminal content $(2.242 \pm 0.077 \mathrm{~g})$, respectively. As for the mean weight of the 20 larvae, it was found that wheat bran $(0.549 \pm 0.043 \mathrm{mg})$ allowed better larval development while ruminal content $(0.272 \pm 0.021 \mathrm{mg})$ allowed a relatively low larval weight gain. Preliminary nutritional analyses of maggots have shown that maggots raised on rice husks showed the highest protein content (42.46\%) while those raised on millet bran only showed a protein content of $37.85 \%$. Concerning the fat content, the maggots produced on wheat bran were the richer $(20.84 \%)$ while those produced on brewer grains had lower lipid level (13.20\%). These results indicate that plant-based materials seem to be the most suitable for the insect development and that millet bran, locally produced and available, could be a suitable candidate for rearing $M$. domestica in Niger. Other analyses are still in progress (i.e. fatty acid compositions) to evaluate the quality of the insects produced and their potential integration in chicken nutrition. Multi-ingredient diets are also planned in order to optimise the growing and potentially the nutritional value of this species. 


\section{Harvesting \& traditional role of eating insects/ sustainable consumption $\&$ food security}

\section{Exploring the governance of an edible insect trade chain through the lens of mopane worms in southern Africa}

\section{J.G. Sekonya* and R.P. Wynberg}

Department of Environmental and Geographical Science, University of Cape Town, Private Bag X3, Rondebosch, 7701, South Africa; gjsekonya@gmail.com

The harvesting and consumption of mopane worms (Imbrasia belina) is a popular livelihood mainstay of rural households across the distribution range in southern Africa. The popularity of the caterpillar is partly due to its ties within local diets, cultures and customs and its revered nutritious value, especially for impoverished households. The mopane woodlands in Botswana attract thousands of harvesters from across the country and prospective exporters from South Africa and Zimbabwe. The harvests support a thriving market for mopane worms worth an estimated US\$39-59 million per year. The commercialisation of mopane worms in Botswana has resulted from multiple factors, including the need to earn income, dwindling outbreaks in neighbouring countries and increasing demand from the South African market. Increasing commercialisation, unprecedented harvesting pressure, habitat destruction, land degradation, and climate change are threatening the sustainability of mopane worm populations, with significant implications for resource governance. Using multi-sited ethnography and a case study approach, we investigated the institutional and regulatory frameworks within which mopane worm livelihoods are situated and the extent to which governance approaches constrain or promote the informal cross-border trade. Actor perspectives revealed significant governance challenges that impact their ability to access harvesting sites and markets, including regulatory overburden, overlapping institutional mandates and a high cost of compliance cost significantly. The incapacity of regulatory institutions to enforce rules inadvertently encouraged corruption and cross-border smuggling to circumvent costly compliance requirements. The discrimination of non-nationals at harvesting areas in Botswana and markets in South Africa were the source of retaliatory exclusions which hindered the aspirations of cross-border traders. Moreover, increasing commercialisation led to an increase in elite capture as well as the flouting of customary harvesting rules and norms. Logistical, financial, and administrative burdens on harvesters, traders and exporters undermined the appetite for compliance with established customary practices, laws, and statutory rules. The ineffective governance and regulation of mopane use, and trade have the potential to undermine the sustainability of mopane worm populations and the livelihoods of harvesters, exporters, and traders in the region. 


\title{
Communities, insects and sustainable food production systems - experiences of insect gatherers in Uganda
}

\author{
L. Binego \\ Coventry University, Ryton-on-Dunsmore, CV8 3LG, United Kingdom; lilianebinego@coventy.ac.uk
}

The urgency to achieve food security and improve nutrition, and the impact of climate change at global level have led to an increased focus on establishing sustainable food production systems. Throughout history, for some communities, insects have contributed to food security and the management of a shared environment. Traditionally, certain insect species are identified as suitable for human consumption as direct food or used in livestock feed, cropping processes, and in sustaining the agrobiodiversity as pollinators. At least two billion people eat about two thousands one hundred and forty insect species worldwide. For these consumers, insects are part of their traditions, food culture and livelihoods. There is the need to recognise insect gathering as part of these communities' food production systems. This paper will address the role of selforganised groups of insect gatherers as a driving force in developing and sustaining food production systems in Uganda. Using a participatory action research approach, data collection involved a community mapping framework and semi-structured interviews to establish the typology of grasshopper harvesting actors and activities in three Ugandan communities; and to identify the socioeconomic and environmental value of gathering activities and harvested grasshoppers. The study sample involved three hundred and forty research participants, with data collection spanning across three consecutive harvesting seasons. The emerging themes point to traditions and cultural practices as key facets that underpin self-organised community of grasshopper gatherers in terms of responsibilities, accountability, rules and norms that govern the grasshopper gathering activity in the three communities. Collected data illustrates ways in which key variables, e.g. gender and socioeconomic status intersect with gatherer status, gathering activities and gathering environment; shape gatherers' understanding of how gathering activities are assessed, interpreted and negotiated at different stages of the process, and influencing factors of ownership, access, value setting, amenities, socioeconomic networks. This paper argues that as the investigated communities recognise the significant contribution of insect gathering to food security, wellbeing, and local economy, in locations where entomophagy is a common practice, insect gathering forms an integral part of local food production systems. The paper further argues that the blending of traditions, culture, norms and local knowledge shapes localised and specific conditions that influence insect gathering framework, power sharing, and establishment of individual rights and related responsibilities toward developing and sustaining insect gathering within local food production systems.

\section{Promoting the production and transformation of edible insects in West and Central Africa}

\author{
P. Le Gall ${ }^{1 *}$, F.M. Muafor ${ }^{2}$, S. Mezdour ${ }^{3}$, I. Le Mintier ${ }^{4}$, G. Flutsch ${ }^{5}$, M. Vaxelaire ${ }^{6}$ and M. Ramnuth ${ }^{7}$ \\ ${ }^{1}$ Evolution Génomes Comportement Ecologie CNRS, IRD, Univ. Paris-Saclay, 91198 Gif-sur-Yvette, France; ${ }^{2}$ Living Forest \\ Trust, BP 1857, Yaounde, Cameroun; ${ }^{3}$ AgroParisTech UMR GENIAL, 31326 Castanet Tolosan, France; ${ }^{4}$ ARMELL La Motte- \\ Basse, Le Gouray, 22330 Le Mene, France; ${ }^{5}$ Planète Cétoines, 91940 Gometz le Chatel, France; ${ }^{6}$ Action Against Hunger, \\ 14 boulevard Douaumont, 75017 Paris, France; ${ }^{7}$ Experts Solidaires Agropolis, 2 Bd de la Lironde, 34980 Montferrier/Lez, \\ France; philippe.le-gall@ird.fr
}

The accelerated social changes that accompany globalisation, deforestation and population growth requires sustainable ways of producing edible insects. Despite the recent development of production through rearing in many Western and Eastern countries, the consumption in Africa is still based on the use of insects collected in the wild even if the quantities involved are important. Most of the available data concern the traditional use and the nutritional quality of consumed species, but local to international markets are poorly documented. Here, we describe pilot farms and studies of the value chain of edible insects, which have been developed in 3 African countries. In Cameroon, the African palm weevil (APW) is an important food and economic resource. In an attempt to promote the use of the APW as an alternative source of protein, a farming design for small-scale farmers to produce these edible larvae was developed through the collaboration of IRD and LIFT with people already engaged in the APW market. This farming system is far less destructive to the raffia ecosystem and more productive than collection in the wild. In Benin, the project led by Experts-Solidaires aims to build an experimental farm and processing unit dedicated to the production of two African crickets, Gryllus sigillatus and Gryllus bimaculatus, within the Songhaï Center in Porto-Novo. The objective is to offer a variety of protein-enriched foods based on insect meal to fight against malnutrition. The project aims to spread the model to independent farmers. Saturniidae caterpillars figured the most consumed insects in Rep. of Central Africa. The goals of the project leaded by ACF are to described and consolidated the existing market of edible caterpillars and then to document the ways to strengthen the production and the consumption of this valuable source of proteins through insect rearing and/or support of the host plant and caterpillars populations. Insects belong to the food tradition of African populations. To ensure the sustainability of the food chain value of edible insects efforts should develop rearing and rationalisation of the market. New products based on insect meal should be proposed to the growing urban populations to maintain their use of traditional foods and increase product quality. 


\title{
Edible insects for child nutrition
}

\author{
C. Kipkoech ${ }^{1 *}$, J. Kinyuru ${ }^{1}$ and N. Roos ${ }^{2}$ \\ ${ }^{1}$ Jomo Kenyatta University of Agriculture and Technology, Department of Food Science and Technology, P.O. Box 62000- \\ 00200, Nairobi, Kenya; ${ }^{2}$ University of Copenhagen, Department of Nutrition, Exercise and Sports, Rolighedsvej 26, 1958 \\ Frederiksberg C, Denmark kipkoechcarolyne@gmail.com
}

Rearing of crickets has gained interest due to high nutrients in crickets. Moderate wasting and stunting common in children are a major contributor to mortality in childhood, attributed to subclinical infection, altered gut microbiota and intestinal inflammation. In rural household, lack of access to proteins and minerals for children is the biggest contributor to malnutrition. To access the impact of cricket-based porridge on child nutrition, a randomised controlled trial ISRCTN10920322 was set up. Children were fed for six months and anthropometry measure were taken at baseline and repeated monthly for six months. There were $37.7 \%$ males and $62.3 \%$ females enrolled in the study. Throughout all the follow-up period, $70 \%$ of the children were under 5 years of age. The mean age at baseline ( 53.37 months) was significantly different from age at end line (58.23 months) $P=0.00$. WHZ at baseline (-1.52) was significantly different from WHZ at end line (0.91) $P=0.00$. WAZ at baseline was positively correlated with WAZ at end line (0.59). The highest correlation (0.97) was seen between baseline and end line HAZ. There was positive change in child anthropometry indicators showing positive growth in children. This clearly indicates a positive growth in children during the intervention period. Training parents on cricket rearing for household consumption is likely to bring a positive change in poor household by providing a cheap source of proteins and minerals. There is need to support rural farmers to rear crickets to alleviate malnutrition.

\section{Bamboo worm harvesting in Myanmar: more benefits or threats?}

\author{
M. Thu Thu Aung" and J. Dürr \\ Center for Development Research (ZEF), University of Bonn, Genscherallee 3, 53113 Bonn, Germany; \\ myintthuthuaung@gmail.com
}

In Myanmar, different kinds of insects are consumed depending on the region. In Eastern Shan State, bamboo caterpillars (Omphisa fuscidentalis) are abundant and highly esteemed for human consumption. The larvae predominantly infect the Wabo bamboo (Dendrocalamus brandisii), which traditionally is cut down for harvesting them. This study aims to investigate the socio-economic benefits and ecological threats of bamboo worm harvesting in local communities. Interviews with value chain actors were conducted in Kengtung district, using a structured questionnaire. Results show that the bamboo worm is a traditional, seasonal food, eaten both as meals and as snacks. Its main season is from September to December; however, fried bamboo worm is available at markets throughout the year. It is marketed not only regionally, but also on foreign markets such as China and Thailand. In recent years, demand and prices have been increasing, and this was reflected in a rising number of bamboo harvesters in the villages. Harvested quantities vary depending on the skill of the hunter, time spent and method of harvesting. There are two types of harvesting: one is making a hole on the internodes exactly where the bamboo worm lives, and the other is cutting down the entire tree. The latter is the most widely used technique for commercial collection because it is less time-consuming. Skilled hunters get around $10 \mathrm{USD} / \mathrm{kg}$ and can gain up to USD 1,600 per season. The number of actors in the value chain varies from two to six depending on the location of the villages, economic condition of the hunter, market accessibility and distance to markets. Harvesters get the highest share of around $39 \%$ of consumer price, it is followed by retailers with $38 \%$. Whereas local collectors participate with $15 \%$, and wholesalers with $8 \%$. In short, bamboo worms generate not only protein-rich food but also job opportunities as well as income in poor regions of Myanmar. Along with the rising number of hunters, bamboo forest conservation is needed. Strategies for bamboo forest preservation are needed to ensure the sustainability of bamboo worm harvesting. 


\title{
The market of edible caterpillars in the Republic of Central Africa
}

\author{
M. Vaxelaire ${ }^{1^{*}}$, F.J. Muafor ${ }^{2}$, S. Mezdour ${ }^{3}$ and P. Le Gall ${ }^{4}$ \\ ${ }^{1}$ Action Against Hunger, 14 boulevard Douaumont, 75017 Paris, France; ${ }^{2}$ Living Forest Trust, BP 1857, Yaounde, Cameroun; \\ ${ }^{3}$ AgroParisTech, 31326 Castanet Tolosan, France; ${ }^{4}$ Evolution, Génomes, Comportement \& Ecologie, CNRS, IRD, Univ.Paris- \\ Sud, Université Paris-Saclay, 91198 Gif-sur-Yvette, France; mvaxelaire@actioncontrelafaim.org
}

Edible caterpillars are the most consumed insects in Central African Republic (CAR). This informal sector ensures food security, livelihoods, and an important income for the most vulnerable population during the collection season (July to midSeptember). Before the crisis of 2013, the commercialisation was done directly between collectors and vendors, to reach the consumers of the cities. During the crisis, insecurity and political destabilisation led to the disappearance of certain sectors. This was not the case of the caterpillar value chain, which had high demand because of the scarcity of beef. The incorporation of wholesalers, a new contributor into the value chain, has ensured the market. To find out the current situation of the value chain, the author conducted focus groups and semi-structured surveys with the various actors of the chain as well as followup the main markets of the city of Bangui. A survey amongst 405 inhabitants of Bangui and Bossangoa allowed to understand consumption trends and validated the possibility of exploring new processing activities (flour and derivate products such as biscuits): $95 \%$ of respondents declared consuming caterpillars, in majority during collection season (caterpillars are bought alive). In that period, they consume caterpillars up to 4 times a week. Dried and smoked caterpillars consumption is less frequent but $65 \%$ of respondent declared being interested by processed products such as biscuits and donuts. 100 FCFA for biscuits is an acceptable price for $96 \%$ of them. This value chain has demonstrated remarkable social and economic resilience to conflict as opposed to the beef sector, as both have seen a reduction in trading volumes and currently only the caterpillar industry has returned to the pre-crisis stage. The value chain proved to be profitable for all actors, especially for collectors/ smokers because of the very limited initial investment. In a context of extended crisis like that of the CAR, supporting the caterpillar sector is relevant because the investment is minimal to allow the creation of jobs and significant income for those who participate, as well as a source of good quality protein. On top of that, women, organised for commercialisation, dominate the value chain, offering them opportunities for income generation. However chemical analysis showed concerning amounts of polycyclic aromatic hydrocarbons, far above the amounts tolerated by European legislation, opening the field to explore low-cost improved smoking techniques.

\section{Edible caterpillars and insects in North Kivu: a potential asset for the development and strengthening of community forestry}

\author{
S. Person ${ }^{1 *}, M$. Nsase $^{2}$ and T. Twendi ${ }^{3}$ \\ ${ }^{1}$ Forest Goods Growing, Montpellier, France; ${ }^{2}$ FODI (Forêt pour le Développement Intégral), Walikale, Democratic Republic of \\ Congo; ${ }^{3}$ SENAD (Sauvons les Êtres et Nature en Détresse), Pinga, Democratic Republic of Congo; forestgoodsgrowing@gmail.com
}

North Kivu is one of the twenty six provinces of the Democratic Republic of Congo (DRC) located in the east of the country. Forests cover almost $70 \%$ of the area. Anxious to contribute to the sustainable management of forest resources while ensuring local development of rural communities, the Congolese government supports the development of community forestry by creating forest concessions for local communities (CFCL). Among the various forest products exploited, caterpillars and other edible insects have an important place there and their development could be an asset for local development. The inventory of edible caterpillar species was done on the basis of surveys carried out during the development of CFCL management plans and the inherent participatory process in Walikale area. Almost fifteen commonly consumed edible species have been identified. Some are particularly appreciated and sought after like the Milanga caterpillar (in kilega). Taxonomic identification of the majority of them is underway. Harvesting is done in the traditional way by collecting caterpillars on the ground, but also by certain destructive practices such as tree cutting. Some of them can occasionally be sold on local markets. But our surveys have shown that the resource is becoming scarce and certain species such as the tukumombo (Imbrasia epimethea) have almost disappeared. Several factors can explain this rarefaction: overexploitation of the resource, deforestation and in particular the killing of host species and finally the possible impact of climate change. There do not seem to be any practices promoting the reproduction of the resource, such as the maintenance of host tree species in the cleared plots. There are also no rudimentary farming practices. Additional research remains to be carried out to estimate the resource more precisely: the precise taxonomic identification of species, and their ecology, and also on host tree species. To allow edible insects to contribute to the socioeconomic development of communities, certain actions deserve to be carried out within the framework of community forestry actions and the development of forest management plans: (1) mobilisation of communities in the preservation of the resource through the adoption of good practices (harvest quotas, harvesting period, defended areas, etc.) and the preservation and / or cultivation of host trees; (2) better valorisation of the resource by improving processing practices and storage. 


\title{
Entomophagy in Australia: could insects solve the impending food security challenge?
}

\author{
R. Ponce-Reyes ${ }^{1 *}$ and B. Lessard ${ }^{2}$ \\ ${ }^{1}$ CSIRO Land \& Water, EcoSciences Precinct, 41 Boggo Rd, Dutton Park 4102, Queensland, Australia; ${ }^{2}$ CSIRO National \\ Research Collections Australia, Clunies Ross Street, Acton, ACT 2601, Australia; rocio.poncereyes@csiro.au
}

Producing sufficient protein to feed our growing population is a global challenge that will not be met using current food sources alone. Currently the dominant protein sources in the western world have high environmental and production costs. In contrast, eating insects promises significant health, cultural and environmental benefits. Entomophagy is an emerging, rapid growth market in Europe, and the USA, however, Australia lags behind with only a handful of early adopting local industries farming insects, even though eating insects has a long tradition in Australia. In August 2019, we held the 'Developing Australia's edible insect research and industry to improve environmental, health and cultural outcomes' symposium in Brisbane. We brought together leading scientific and traditional knowledge of native Australian edible insects. The purpose of the symposium was to promote their inclusion in our diet, and drive commercialisation opportunities. During the symposium we identified the research priorities of edible insects in four major topics: culture, environmental benefits, health and diet and commercialisation. In this talk we will present our findings, including a roadmap to farming novel Australian insect species and implementing insect-derived protein into our diet.

\section{Insects for peace: ex-combatants as smallholder insect producers in post-conflict Colombia}

\author{
K.B. Barragán-Fonseca ${ }^{1 *}$, K.Y. Barragán-Fonseca ${ }^{2}$, G. Verschoor ${ }^{3}$, J.J.A. van Loon $^{2}$ and M. Dicke ${ }^{2}$ \\ ${ }^{1}$ Departamento de Producción Animal, Facultad de Medicina Veterinaria y de Zootecnia, Universidad Nacional de Colombia, \\ 111321 Bogotá, Colombia; ${ }^{2}$ Laboratory of Entomology, Wageningen University, P.O. Box 16, 6700 AA Wageningen, the \\ Netherlands; ${ }^{3}$ Sociology of Development and Change Group, Wageningen University, P.O. Box 8130, 6700 EW Wageningen, \\ the Netherlands; kbbarraganf@unal.edu.co
}

Black soldier fly (BSF) is a nutritious feed component for livestock with high protein levels. BSF can be reared on a wide range of organic residual streams. This allows for local production within a circular agriculture, decoupling livestock production from import of expensive feed components such as fishmeal or soymeal. Rearing of BSF can be done by smallholder farmers, thus contributing to their livelihood, economic sustainability and social status. Smallholder farmers contribute importantly to food security, which is a prerequisite for a stable society. In countries recovering from conflict, agricultural development should focus on restoring food production by smallholder farmers, improving their socio-economic position, thereby contributing to sustainable development goal 16 (SDG16, peace and justice). In the last sixty years, Colombia has been involved in an armed conflict that officially ended in 2016 with the signing of the peace agreement after four years of negotiations between the Colombian government and The Revolutionary Armed Forces of Colombia (FARC-EP). Therefore, supporting agricultural projects and strengthening institution building and food security has become an imperative issue, where academia has a major role. Here, we present how BSF can be a sustainable fish feed to reduce the costs of commercial feed used in the production of tilapia and an example of reintegration of ex-combatants as smallholder insect producers in post-conflict Colombia. 


\title{
Improving traditional insect trapping techniques to enhance crop protection and supply of edible insects in East Africa
}

\author{
J.P. Egonyu ${ }^{1 *}$, F. Sengendo ${ }^{1,2}$, M. Chemurot ${ }^{2}$, C.M. Tanga ${ }^{1}$ and S. Subramanian ${ }^{1}$ \\ ${ }^{1}$ International Centre of Insect Physiology and Ecology (icipe), P.O. Box 30772-00100, Nairobi Kenya; ${ }^{2}$ Makerere University, \\ P.O. Box 7062, Kampala, Uganda; pegonyu@icipe.org
}

Harvesting edible insects from the wild has been traditionally practiced across the world since time immemorial. However, little has been done to improve rudimentary traditional techniques used for harvesting edible insects. On a flipside, some of the common edible insects such as palm weevils, termites and locusts are renowned devastating crop pests. Tailoring technological innovations toward the dual purpose of trapping pestiferous insects as a crop protection strategy as well as amassing edible insects for food, feed and other uses is an appealing sustainable approach. To this end, the International Centre of Insect Physiology and Ecology (icipe) with its partners has improved existing trapping techniques of the African palm weevil (Rhynchophorus phoenicis) and the longhorned grasshopper (Ruspolia differens). Using traps baited with the aggregation pheromone of $R$. phoenicis (3-methyl-octan-4-ol) and a plant based kairomone (ethyl acetate), hundreds of adult beetles were trapped from wild and cultivated palms in Uganda and Kenya, thus significantly reducing their damaging potential on the plants. Samples of live adult $R$. phoenicis were used to initiate captive rearing of the insect at icipe for human consumption. We also optimised the traditional technique for harvesting $R$. differens in East Africa by: (1) modifying collection barrels to improve retention of catches and filter out harmful and/or non-target insects; and (2) demonstrating cost-effectiveness and sustainability of light emitting diode bulbs over traditionally used mercury bulbs that consume twice more energy. The implications of the mercury bulbs in environmental pollution is discussed. Our future efforts will focus on scaling out these innovations and adapting them for sustainable management of other crop devastating edible insects.

\section{Anthropoenthomophagy in Mexico: a review of its history, current situation and perspectives}

\author{
J.M. Pino Moreno \\ Biology Institute UNAM (National Autonomous University of Mexico), Department of Zoology, Entomology Laboratory, \\ Ap. Postal 70-153, Mexico City, Mexico; jpino@ib.unam.mx
}

Since 1974, in the Biology Institute, UNAM, Dr Julieta Ramos-Elorduy initiated the research project Insects as a source of protein for the future. I review the most salient aspects studied so far; these include historical research about edible insects in ancient Mexico, field research that includes interviewing people in rural areas so as to gain knowledge about, detect and collect edible insects, research about the common names of 132 species, contribution to the National Collection (in the Entomology Laboratory we have mounted, labelled, identified, catalogued and incorporated to the collection, situated in the same institute, 560 species and we have generated data bases and catalogues), culture (for example, rustic culture of ahuahuatle, axayacatl and escamol, semi industrial of silkworm and industrial of flour yellow worm; this process has also enabled the generation of organic fertilisers), record of commercialisation sites (in certain roadsides, tianguis, markets, restaurants, etc.), analysis of nutritional value (in collaboration with the Animal Nutrition and Biochemistry Department of the Faculty of Veterinary Medicine and Animal Husbandry, UNAM, since 1978 we have applied the official techniques of the AOAC to quantify amino acids, minerals, vitamins and fatty acids, as well as the nutritional value of the hosts), development of new gastronomical strategies to enhance palatability and acceptance, included the application of food technology to manufacture insect brittles, cookies, sauces, sweets, bread, desserts and ice creams, that have been subjected to microbiological analyses and whose shelf life and sanitary status have been evaluated, development of different types of animal feed (for example, we have tried with flour yellow worm, flies, grasshoppers, cockroaches, escamol, bees and axayacatl, in the diet of Wistar rats, fish, pigs and different types of poultry), and development of a legislation that includes recommendations about extraction, exploitation, management, production, distribution and commercialisation. With all this information we have so far published many research articles in national and international scientific journals, as well as books and book chapters, and we can say that insects in Mexico are an element of cultural identity, as in the case of the jumiles of Taxco, Guerrero. Insects are managed as a natural renewable resource and have become a significant economic activity in different parts of the country; this is why even some entrepreneurs already consider them in the creation of alimentary micro business and, as such, as a source of employment. 


\title{
Diversity and nutritional composition of edible insects in Sub-Saharan Africa
}

\author{
Z.T. Hlongwane", R. Slotow and T.C. Munyai \\ School of Life Sciences, University of KwaZulu-Natal, Private Bag X01, Scottsville 3209, South Africa; \\ nolwazihlongwane20@gmail.com
}

Insect are consumed as food in different parts of the world, and their consumption is not a new phenomenon, as this played an important role in food security when people were primarily hunter-gathers. Edible insects are an important natural resource that has a potential in contributing to food security, and edible insects do not only play an important role in traditional diets, but are also an excellent source of protein in traditional dishes in Africa. To explore the diversity and nutritional status of edible insects in Africa, we conducted a systematic review using the Thomson Reuters' Web of Science and google scholar database, resulting in eligible 98 papers. A list of 212 edible insect species from eight orders (41\% Lepidoptera, 23\% Orthoptera, 15\% Coleoptera, $12 \%$ Blattodea, $4 \%$ Hemiptera, and Hymenoptera, Diptera, and Mantodea all $<1 \%$ ). These insects are rich in protein, fats, and fibre. The highest protein content was reported for Lepidoptera (range: 20-80\%) and Orthoptera (12-73\%). Coleoptera had the highest carbohydrate content (7-54\%), while order Lepidoptera had the highest fat content (10-50\%). Iron content was highest in Orthoptera $(0.3-910 \mathrm{mg} / 100 \mathrm{~g})$. Considering the excellent source of nutrition, and potential socioeconomic benefits, from edible insects, they can contribute strongly to improved food security and rural development in developing countries. In addition, edible insects can be used as a sustainable food source to combat food shortages in future, for example, providing resilience during times of drought or other climate stressors.

\section{Nutritional value of edible termites Macrotermes falciger collected from Lusaka District, Zambia, a potential agent against Malnutrition}

\author{
M.C. Chulu ${ }^{1 *}$, L.T. Muungo ${ }^{2}$ and A.B. Gono ${ }^{2}$ \\ ${ }^{1}$ Levy Mwanawasa Medical University Pharmacy Department, P.O. Box 33991, Lusaka, Zambia; University of Zambia, \\ School of Medicine, Department of Pharmacy, P.O. Box 50110, Lusaka, Zambia; chulumartha@yahoo.com
}

Winged termites (Macrotermes falciger) are a highly relished insect, which is common in Zambia during the rainy season. The winged termites are de-winged, roasted and salted for use as a snack or in combination with a carbohydrate. The main objective of this study was to evaluate the nutritional components of the termites with a view to revealing any possibility of its use as a base for the formulation of new complementary foods as possible means of combating malnutrition. The winged termites were collected from Lusaka District and roasted for ten minutes, de-winged. The dried sample was milled with a blender into a fine powder and transferred to a $500 \mathrm{ml}$ wide reagent bottle labelled as 'Roasted sample' and kept at room temperature in a dark place until when needed for analysis. The proximate, mineral, vitamin $C$ and fatty acid composition of the termites were analysed according to the Association of Official and Analytical Chemist (AOAC) methods. Roasted $M$. falciger contained moisture (6.0 $\pm 0.02 \%)$, ash $(5.7 \pm 0.05 \%)$, crude protein, $(23.1 \pm 0.00 \%)$, crude fat $(46.5 \pm 0.01 \%)$, crude fibre $(2.2 \pm 0.48 \%)$, energy $(591 \mathrm{kcal} / 100 \mathrm{~g})$, ascorbic acid $(1.1 \mathrm{mg} / 100 \mathrm{~g})$, sodium $(118.7 \pm 0.02 \mathrm{mg} / 100 \mathrm{~g})$, potassium $(220.4 \pm 0.06$ $\mathrm{mg} / 100 \mathrm{~g})$, calcium $(81 \pm 0.03 \mathrm{mg} / 100 \mathrm{~g})$ zinc $(5.3 \mathrm{mg} / 100 \mathrm{~g})$, magnesium $(132 \mathrm{mg} / 100 \mathrm{~g})$, manganese $(147.5 \pm 0.01 \mathrm{mg} / 100 \mathrm{~g})$, selenium $(22.2 \mathrm{mg} / 100 \mathrm{~g})$, phosphorous $(81 \mathrm{mg} / 100 \mathrm{~g})$. The total fatty acid content was $45.3 \mathrm{~g} / 100 \mathrm{~g}$ and the individual fatty acids were oleic acid $(26.37 \mathrm{~g})$, pentadenoic acid $(0.09 \mathrm{~g})$, palmitic acid $(12.3 \mathrm{~g})$, palmitoleic acid $(0.98 \mathrm{~g})$, myristic acid $(0.31$ g), margaric acid $(0.14 \mathrm{~g})$ and stearic acid $(5.17 \mathrm{~g})$. The high fat content of the termite was made up mainly of unsaturated fatty acids (60.3\%). The above results suggest that the winged termite, $M$. falciger has nutritional and pharmaceutical potential and can be used to combat protein energy related malnutrition prevalent in Zambia if used as part of complementary foods. 


\title{
Perception, marketing \& economics
}

\section{Overall acceptability of fillets of rainbow trout fed diets with increasing levels of Tenebrio molitor larva meal}

\author{
A. Brugiapaglia ${ }^{1}$, S. Bellezza Oddon ${ }^{1}$, I. Biasato ${ }^{1}$, M. Renna ${ }^{2}$, G. Chemello $^{1}$, C. Caimi ${ }^{1}$, A. Schiavone ${ }^{2}$, F. Gai ${ }^{3}$ and \\ L. Gasco ${ }^{1 *}$ \\ ${ }^{1}$ Department of Agricultural, Forest and Food Sciences, University of Turin, Italy; ${ }^{2}$ Department of Veterinary Science, \\ University of Turin, Italy; ${ }^{3}$ Institute of Science of Food Production, National Research Council, Largo P. Braccini 2, 10095 \\ Grugliasco (TO), Italy; laura.gasco@unito.it
}

Insect meals are promising candidates as protein source in aqua-feeds. This study evaluated the overall acceptability of growout rainbow trout fed increasing dietary levels of defatted Tenebrio molitor larvae meal (T) and precisely: 0\% (T0), 5\% (T5), $10 \%$ (T10), and 20\% (T20). At the end of the trial (154 days), fish fillets were vacuum-cooked (water bath, $75^{\circ} \mathrm{C}, 20 \mathrm{minutes}$ ). A total of 85 untrained consumers were asked to evaluate the sample overall acceptability by the use of the 9-point hedonic scale. Afterwards, the consumers were asked to motivate their judgment choosing one of the following organoleptic characteristics: odour, taste or texture. A mixed ANOVA model with overall acceptability as dependent variable, dietary treatment as fixed effect and consumer as random effect, was performed. Tukey's multiple comparison test was used to determine significant differences $(P<0.05)$ among experimental groups. The acceptability scores given by each consumer were then converted into ranked data by assigning rank order numbers to the evaluations. Ranking data were analysed with the non-parametric Friedman's test. Finally, the numeric hedonic scores were classified into two categories as follow: $\leq 5$, representing negative ratings ('dislike extremely' to 'neither like nor dislike') and $>5$ representing positive ratings (like slightly to like extremely). Once the data were categorised a correspondence analysis was performed involving the organoleptic characteristics chosen by consumers to motivate their judgement. The results of the affective test showed that mode of T5 group showed the highest value ( 8 = like very much). The average acceptability scores of the trout fed on T ranged from 5.88 (T20) to 6.93 (T5), which corresponds to 'like slightly' and to 'like moderately' according to the 9-point hedonic scale. On average, about the 75\% of the consumers rated acceptable the meat of the four groups. ANOVA performed on mean hedonic ratings showed that fillets from T5, and T10 groups were preferred in comparison with T20 group. No differences were reported for all fish groups fed insect meals against T0. The Friedman's test confirmed the ANOVA results. In fact, T5 and T10 groups were preferred in comparison with T20 group. Therefore, even if all groups were well accepted by consumer, results highlighted that a partial replacement of fish meal with $\mathrm{T}$ improves the overall acceptability of the product as indicated by correspondence analysis. 


\title{
Role of culture in the acceptability of insects as food
}

\author{
A. Marie ${ }^{1 *}$, G. Pantin-Sohier ${ }^{1}$ and C. Gallen ${ }^{2}$ \\ ${ }^{1}$ Laboratoire GRANEM, IAE Angers, 13 allée François Mitterrand, 49036 Angers Cedex 01, France; ${ }^{2}$ Laboratoire LEMNA, \\ IAE Nantes, chemin de la Censive du Tertre, BP 52231, 44322 Nantes Cedex 3, France; agathe.marie@etud.univ-angers.fr
}

Entomophagy, the practice of eating insects, is recognised as a sustainable food solution to face the rising population, both in terms of protein intake and environmental footprint. Even if it tends to rise in Western countries, entomophagy is hampered by Westerners' mental representations of insects considered inedible. The perceived edibility of a product varies from one individual to the next according to different contextual factors, primarily the individual's cultural system. Indeed, cultural norms indicate how to mentally categorise a food and determine its sensory properties. Culture therefore explains why it is so difficult to introduce new food products into many of the world's regions. In addition, disgust is one of the major reasons identified by anthropologists for the rejection of a food. It corresponds to the repulsion felt at the idea of incorporating a substance and can be explained by the fear of contamination. A way to reduce this food disgust is familiarisation, a repeated exposure to a product. Familiarisation decreases the aversion towards a product, also resulting in greater appreciation and acceptance with a raise of the willingness to try it. Then, it is important to consider the level of familiarity with entomophagy within a country when studying its acceptability. This acceptability is also influenced by consumers' attitude, a major predictor of behaviour in Marketing studies. The aim of this study is to determine the role of culture in the acceptability of entomophagy with three measures: the proportion of participants who have already eaten insects (familiarity), their food disgust level and their attitude towards entomophagy. To test our hypothesis, we decided to apply measuring scales developed in consumer behaviour (Food Disgust Scale, Entomophagy Attitude Questionnaire) on a sample of French consumers and compared our results with those obtained in other countries: inside (Denmark, England, Germany, Italy, Spain, Sweden, Switzerland) and outside (Australia, USA) Europe. The study conducted on 110 participants reveals cultural differences on several points. First, the proportion of participants who have already eaten insects showed various levels of familiarisation among Westerners. Then, results indicated different food disgust levels considering the country of the participants. Finally, the attitude towards entomophagy was different for each of the three dimensions of the scale: disgust, interest, feeding animals. Hence, by influencing familiarisation, food disgust and attitude, culture also impacts the acceptability of insects as food.

\section{Bugs for the future: a step-by-step education guide for learning about sustainability with insects at home \& in schools}

\author{
K. Unger \\ Livin Farms HK Ltd, 38 Lok Ku Road, 2 F Casey Building, Room 06, Sheung Wan, Hong Kong; katharina@livinfarms.com
}

There are still several hurdles for insects to become a staple protein source for humans and animals. Although consumers seem to have a good understanding about the environmental benefits of insects as a protein source, there is still a lack of education when it comes to eating insects or raising them as pet food or animal feed. Also, the so called 'Yck Factor' is still a known barrier for market entry. Many of the prejudices could be overcome if there was more education on the 'Why' of the insect sector. Along the lines of a well-known proverb 'A tree must be bent while it is young', young people can be a critical benchmark for insects to reach wide acceptance as a protein source in the near future. Furthermore, as sustainability is becoming an obligatory lesson in some national curricula, there is opportunity for insects to act as important messengers of sustainability learning. Livin Farms has developed a mini insect farm ('Hive Explorer') and accompanying education materials and employed them in several workshops and classroom sessions for students from Kindergarden to University, starting at age 5 . In the work process with more than 20 educational institutes and educators, mostly in Asia and Europe, several learnings were collected. A step-by-step approach was developed and utilised for hands-on learning processes on a variety of topics relevant to international curricula. The topics range from learning about the biology of insects and their position in the ecosystem, to food waste and food systems, emissions and climate change down to nitrogen cycles and plant growth. Focus was on the closed loop principle that insects can support. For example, when feeding them kitchen wastes from home kitchens or cafeterias, then using the droppings as fertiliser for growing plants that are later harvested as edible greens. This process has revealed insights that are relevant for stakeholders across the insect sector, including 'pick up lines', 'battle lines', 'learning lines' and a process structure to use to communicate effectively about insects, create interesting talking points and in consequence, to reach a larger audience. 


\title{
Market opportunities for insects in dog food
}

\author{
S. Floto-Stammen", N. Naranjo-Guevara and J. Janke \\ Fontys International Business School, Tegelseweg 255, 5912 BG Venlo, the Netherlands; s.flotostammen@fontys.nl
}

Insects have been proposed as an alternative high quality, efficient and sustainable food protein source to the conventional livestock feed protein. In the present study, possibilities for the use of insects in the dog food market were identified in order to answer the question whether animal feed manufacturers should take into account the increasing trend of insect-based animal feed and bring a corresponding product onto the market. We are carrying out a qualitative analysis through expert interviews to answer the next questions: (1) What is the current situation on the insect-based pet food market in Germany and the Netherlands? (2) How is the existing pet food item for insects positioned on the market? (3) Why have companies added insect-based animal feed to their range? (5) What are the reasons why dog owners buy food with insects? Four different companies were selected for the interviews: two companies positioned the insect products as a sustainable option, the other two as a hypoallergenic option. The Value Proposition Canvas was used to find out whether the used claims also solve the customer's needs and problems. From our results, we can say that consumers ask for specific allergy diets. A prerequisite for an allergy diet is a single source of a protein that is not common in the diet. The dog food market should be monitored, and further research carried out to identify possible allergies to the protein in insects. With regard to insects as a sustainable option, the actual environmental impact of each company should be assessed separately and examined how the insect supply chain can be built as sustainably as possible. Our research is an orientation tool that companies can use to decide whether this material fits their marketing strategy or whether they may have another product in their portfolio that meets the same requirements as insect-based animal feed.

\section{Potential of increasing the insect production in farms: the case of Northern Ostrobothnia, Finland}

\author{
K. Korhonen ${ }^{1}$, S. Heiska ${ }^{2 *}$, P. Marnila ${ }^{3}$, T. Muilu ${ }^{1}$, M. Mäki ${ }^{3}$ and A.L. Välimaa ${ }^{1}$ \\ ${ }^{1}$ Natural Resources Institute Finland (Luke), Paavo Havaksentie 3, 90570 Oulu, Finland; ${ }^{2}$ Natural Resources Institute \\ Finland (Luke), Yliopistokatu 6 B, 80100 Joensuu, Finland; ${ }^{3}$ Natural Resources Institute Finland (Luke), Myllytie 1, 31600 \\ Jokioinen, Finland; susanne.heiska@luke.fi
}

We studied the potential of increasing insect production among farmers in the province of Northern Ostrobothnia, Finland. The study was conducted as a part of an electric survey carried out in five provinces, in January 2020. Total of 27 responses were received from farmers in Northern Ostrobothnia (response rate 6\%) and 163 responses nationwide (response rate 7\%). The survey data was observed using statistical descriptives and non-parametric Kruskal Wallis Test as well as crosstabulations. Formation of an innovation ecosystem of insect economy has been building up in Finland since 2017, when the Ministry of Agriculture and Forestry accepted insects as food and feed. A few months after legalisation, there were several new insect food products in wide distribution via supermarkets and retail chains. The demand for local cricket powder increased rapidly, as significant players of food industry concurrently launched new insect food products in the market. The number of cricket farmers exceeded 50 in a few months. However, the change in consumer preferences has not been as fast as expected. After a short hype phase, most of the insect food products disappeared from markets. Concurrently, the demand for edible insects decreased. Today, the number of producers, who are actively developing their processes, is estimated to be only fifteen. In the near future, changes in the edible insect market are expected, due to the approval of novel food applications on edible insects, and market expansion to the whole EU. Finland is known as a forerunner in technology solutions for house cricket rearing. Finnish insect food products have also received international attention and some of the products are manufactured for export. With the existing know-how, a rapid increase in production capacity could be possible. The preliminary results indicate that factors hindering farmers converting to insect production relate, in particular, to profitability and distribution networks as well as the investment requirements. Only a few respondents seemed to have clear interest towards insect production, however about a third couldn't tell whether they are interested or not. Even though the importance and prospects of insect production was seen rather on a global scale than on a national or regional scale the insect production sector might arouse more interest among farmers in the future if more information is available for them. 


\title{
Chicken fed insects must still taste like chicken
}

\author{
L.C. Hoffman ${ }^{1,2^{*}}$ and E. Pieterse ${ }^{1}$ \\ ${ }^{1}$ Department of Animal Sciences, Stellenbosch University, Private Bag X1, Matieland, Stellenbosch 7602, South Africa; ${ }^{2}$ Centre \\ for Nutrition and Food Sciences, Queensland Alliance for Agriculture and Food Innovation (QAAFI), The University of \\ Queensland, Health and Food Sciences Precinct, 39 Kessels Road, Coopers Plains 4108, Australia; louwrens.hoffman@uq.edu.au
}

Irrespective of what insect species or form of said species that chickens are fed, the industry expects the chicken to still taste like 'chicken. The insect species evaluated included: Musca domestica (MD), Chrysomya chloropyga (CC), and Hermetia illucens (BSF). The insect larvae had been fed on various substrates and the insects were harvested at different physiological stages, post-harvest treatments in included at different dietary levels.. After slaughtering the following carcass characteristics were compared: carcass weight, dressing percentage, portion weight/ratio, as well as the meat quality parameters: colour, $\mathrm{pH}$, water binding capacity and sensory evaluation by means of a descriptive sensory analyses by a trained taste panel. For MD, the animal (insect) protein-based diets yielded higher live weight and carcass weight with no difference in dressing percentage. The breast muscle and drumstick yield was similar or higher for the animal protein diets whilst the thigh yield was similar or lower for animal protein diets. Typically, the animal protein diets yielded a higher breast muscle yield than the soybean-based diet. For the BSF and CC based diets, there were no significant differences in the carcass characteristics when compared to the commercial soybean diets. For the meat quality, irrespective of the insect species or physiological stage or processing method, there were no differences in either the initial or final $\mathrm{pH}$ of the meat. However, there were slight differences in in the colour measurements: the larvae fed chickens' breast meat was generally lighter and redder. As pertaining to the water binding capacity, the results were inconclusive. For the sensory characteristics, no differences were observed except for the juiciness and sustained juiciness although it was debatable whether these slight differences would be perceived by consumers. In some of the breasts particularly that derived from the $\mathrm{CC}$ fed abattoir waste a slight metallic flavour was noted. This flavour was linked to the possibility that these breasts might contain higher levels of iron; this aspect warrants further research as iron is known to be limited in typical Western diets and if in sufficient quantities, these chicken breasts could be classified as a functional food. Our data indicates that broiler chickens fed insect larvae meal still taste like 'chicken'. More research is required around the post-harvest processing of the insects to ensure that the meat from the broilers receiving these is 'safe' for human consumption.

\section{Acceptance of Dutch and German students towards insects in feed and food}

\author{
N. Naranjo-Guevara", S. Floto-Stammen, M. Fanter, A.M. Conconi and K. Ruseva \\ Fontys International Business School, Tegelseweg 255, 5912 BG Venlo, the Netherlands; nnaranjoguevara@gmail.com
}

The human acceptance of insects in food and feed is highly dependent on social imprint. Despite the increasing interest in the use of insects as food, the acceptance of insect-based foods in Europe is low. Disgust rejection appears to be an obstacle that could be overcome by exposure to food and information about the ecological and nutritional benefits of using insects as food and feed. As the frequency of contacts and information about insect products is quite low in the European region, we are looking for strategies to speed up the process of being ready to accept insects as part of the human diet. Our study initially aims, to examine the motivation behind the willingness to change eating behaviour in more detail. This was done by interviewing 200 Dutch and German Business students at Fontys International Business School in Venlo near the German border. We investigate how factors such as food neophobia (fear and aversion to new foods), health expectations and involvement in sustainability issues, can influence the willingness to use insects as an alternative protein. Second, we investigate whether and how information can influence acceptance. This was achieved by comparing the willingness to eat insects (meal) before and after adding benefits such as the environmental or health effects to the question. Our results help to provide more concrete insight into consumer motivation to accept novel foods that can support a more effective communication strategy for insectcontaining products. 


\title{
It's smart and delicious - how to convince Americans to adopt insect protein
}

\author{
J. Yoon \\ Brooklyn Bugs, 862 Dekalb Ave, \#14, Brooklyn, NY 11221, USA; joseph@brooklynbugs.com
}

America has a need to address how we can change existing negative consumer attitudes toward insect proteins as the global awareness for why we should consume them increases. Brooklyn Bugs tours across the nation and has worked closely with major universities and museums over the past two years in our mission to combat cultural prejudices and fears. We raise awareness and appreciation for edible insects through delicious, creative, and educational programming, and develop meals of up to twelve tasting courses for tens of thousands of people. Our interdisciplinary approach and progressive outreach have led to significant institutional, cultural, and gastronomical advancements, and have been a consistent source of interest by the media that closely follow and report on our work. Both large scale (for thousands of people) and intimate dining events have been produced at Montana State University (2019, 2020), Purdue University (2019), San Diego State University (2019, 2020), Tufts University (2019), Culinary Institute of America (2018, 2019), University of Wisconsin at Madison (2019), the American Museum of Natural History (2017-2020), NY Hall of Science (2018-2020), Natural History Museum of LA (2018, 2019), and the Museum of Fine Art, St. Petersburg (2019). We endeavour to bring Americans into the global conversation about alternative sources of protein in a rapidly changing world. What is the best method for introducing edible insects to a population already predisposed to view them with a sense of disgust? Are different approaches necessary for different demographics? Are appeals to health and a more sustainable environment sufficient? Has there been any success in converting people who were adamant that they would never eat them? We will share our methods and successful outcomes of what ultimately can lead people to eat insect protein for the first time. We will also explore what challenges need to be overcome for the continued growth of the edible insect industry in America.

\section{Bugs in schools: universities using insects to valorise food waste}

\author{
S. Ku \\ Georgia State University, 35 Broad Street, Suite 620, Atlanta, GA 30303, USA; sku2@gsu.edu
}

Business research focuses heavily on increasing consumption with little attention to the devastating effects of the waste it creates. Food waste comprises the largest category of global municipal solid waste. The waste from food produces methane greenhouse gas, which traps heat in the atmosphere and substantially contributes to global warming. Due to decreasing availability of land for landfill usage as well as the environmental costs of burying trash, alternative strategies require urgent and universal attention. Previous literature uncovers that waste management strategies utilising insects require less resources compared with conventional waste management operations. Universities deal with high quantities of food waste on a daily basis. These organisations may or may not be limited in their waste management practices due to size, location, infrastructure, etc. Therefore, the purpose of this research is to utilise university food waste data to determine optimal waste management solutions. We hypothesise that insect waste management is a viable and profound strategy for economic, environmental, and social gains. Specifically, we examine the business opportunity of insect waste management in the context of universities. 


\title{
Understanding farmers' perceived benefits and value-chain governance of insect-based chicken feed in Kenya
}

\author{
A. Okello ${ }^{1,2 "}$, J. Nzuma ${ }^{1}$, D. Otieno ${ }^{1}$ and M. Kidoido ${ }^{2}$ \\ ${ }^{1}$ University of Nairobi, Department of Agricultural Economics, P.O. Box 29053-00625, Kangemi, Kenya; ${ }^{2}$ International \\ Centre of Insect Physiology and Ecology, P.O. Box 30772-00100, Nairobi, Kenya; afreaqokello@gmail.com
}

The high cost of chicken feed due to scarce protein ingredients continues to have adverse effects on both the household and national status of the poultry value chain in Kenya. Feed accounts for more than $60 \%$ of the poultry production costs and this has created a myriad of challenges including diminished feed quality. This situation has forced smallholder poultry farmers, due to the reduced profit margins, to divert their resources to more lucrative enterprises despite the growing regional and global demand for poultry and poultry products. Literature and research show potent backing towards the integration of the black soldier fly larvae to ensure chicken feed is sustainably produced into the future. While such an intervention is important, it is also crucial to elucidate farmers' subjective preferences for improved products to encourage their successful uptake. Hence, this study assessed the perceived benefits and their preferred institutional transformations of the insect-based chicken feed industry among poultry farmers in Kiambu County, Kenya. Results from an analysis of qualitative data from focused group discussions and key informant interviews revealed that farmers expect: affordable and nutritious feed, reduced chick mortality, efficient feed intake, rapid weight gain, improved egg-laying and profit incentives which will be fundamental in filling the food and nutritional gap. This was attributed to the fact that insects are easily available in the ecosystem and have been used in traditional poultry husbandry due to their high protein content. For the insect-based feed industry to repeal the setbacks of the current value chain: decentralisation of quality regulation institutions to county level, penalisation, public and private partnerships to increase sensitisation and use of ICT to verify quality are some of the transformations that would ensure the success of this novel industry.

\section{Online shopping of edible insects in Myanmar}

\author{
M. Thu Thu Aung" and J. Dürr \\ Center for Development Research (ZEF), University of Bonn, Genscherallee 3, 53113 Bonn, Germany; \\ myintthuthuaung@gmail.com
}

Along with the formation of the first civilian government, the telecommunication sector has developed rapidly after 2010 in Myanmar. Since then, the use of cell phones, social media and online shopping are widespread. At the same time, edible insects have become a fashion food. They are sold on traditional open markets, but for the last four years also increasingly online, expanding the access to edible insects. This paper investigates how the online market is developing, what are the main constraints and how consumers perceive this new market outlet. Data were collected from 'Facebook' and through online interviews with sellers and consumers. The study explored that there are at least 42 online sellers who use their personal account or a business page. All vendors provide a country-wide delivery service. Both wild harvested and reared insects are available: wild cricket, giant water bug, beetles and cicadas are accessible seasonally while reared cricket, bamboo worm, silkworm, and palm weevil larvae are available all year long. Although the number of online sellers become more and more, each seller has regular customers. Almost all sellers combine their insect business with other food. The most popular edible insect is the giant cricket. The majority of customers are female and they spend between 10 to 65 USD per year. First time buying is attracted by photos of edible insect preparation and by feedback from other customers, while the price seems to be of less importance. The main factors for becoming a regular client are good taste, right time delivery, quality, freshness, and removal of inconsumable parts. Customers prefer online shopping to traditional marketing. But its drawback is fraud problem which sometimes happens when sellers and buyers are from different towns; buyers then have to make an advance payment, and will not receive the product. Therefore, consumers are afraid of advance payment which is the main hindrance to the development of insect online shopping. Regulations of online shopping, registration of online sellers and the use of reliable delivery companies are needed to overcome this obstacle. 


\section{Factors affecting nutritional value of edible insects and their acceptability}

L. Kouřimská ${ }^{* *}$ and M. Kulma ${ }^{2}$

${ }^{1}$ Department of Microbiology, Nutrition and Dietetics, ${ }^{2}$ Department of Zoology and Fisheries, Czech University of Life Sciences Prague, Kamýcká 129, 165 00, Praha 6, Suchdol, Czech Republic; kourimska@af.czu.cz

Edible insects belong to the possible alternative food source replacing traditional ingredients. With the growing population and increasing demands on world food production, research on this topic has intensified over the last 20 years. However, there is still insufficient information about the factors affecting composition and nutritional value of some edible insect species and about acceptability of insects among the European consumers. Our research is therefore focused on monitoring the factors influencing the nutritional value of edible insects. The effect of developmental stage on the quantity and quality of nutrients in Zophobas morio and Blaberus craniifer was investigated. Superworms at 60, 90, and 120 days of age showed no significant variation in basic nutrient content, and no significant differences were found in protein quality. In contrast, adult cockroaches contained significantly more digestible protein than either small or large nymphs, but of lower quality. Conversely, the lipid quality of superworm larvae decreased with their age. Nutrient contents of subadult and adult Blaptica dubia, Blaberus discoidalis and Blatta lateralis roaches were also compared. Adults contained more crude protein and ash, but less fat than subadults. The effect of sex on the nutritional value and chemical composition were proved on Acheta domesticus adults. The proximate composition was influenced by sex; females contained a significantly higher amount of lipids and fewer proteins and chitin than males. Our other results showed that the proximate composition of Tenebrio molitor was influenced by increased rearing temperature. Larvae reared at $28^{\circ} \mathrm{C}$ had lower protein content, but higher fat and dry matter content, body weight and length than larvae kept at 22 and $25^{\circ} \mathrm{C}$. Our research in the field of acceptability of edible insects investigated that women and younger assessors in the Czech Republic were slightly more open to the consumption of whole baked house crickets than men and older panellists. Of the 98 panellists, 68 were willing to evaluate the sample visually and then by tasting. There were significant differences in overall pleasantness before tasting (40\%) and after tasting (61\%). The preferred methods of culinary preparation of edible insects that the respondents would choose were baking, roasting and frying. All our studies provide a better understanding of the nutritional value of insects over their lifespan and may help to optimise rearing technology as well as a better understanding of consumers' attitudes towards entomophagy. 


\section{Insect production systems/optimisation, engineering, automatation \& security}

\section{Reducing spatial footprint and handling in a black soldier fly bio-waste conversion unit using incubator containers}

\section{B.M.A. Dortmans", T. Kusumawardhani, T. Rahayu and C. Zurbrügg}

Eawag - Swiss Federal Institute of Aquatic Science and Technology, Department of Sanitation, Water and Solid Waste for Development (Sandec), 8600 Dübendorf, Switzerland; bram.dortmans@eawag.ch

At the FORWARD project pilot site in Sidoarjo, Indonesia, a manually operated black soldier fly (BSF) biowaste conversion system has proven that this approach can offer a solution for urban biowaste treatment close to the source of the generated waste - from households and markets. The city of Surabaya has developed two facilities at waste transfer stations, where biowaste from households and markets is currently processed using BSF larvae. The conversion units are setup as six layers of six plastic crates (each $61 \times 43 \times 15 \mathrm{~cm}$ ) on a pallet. Each layer of crates is separated from the other by a frame providing a ventilation gap of $8 \mathrm{~cm}$. During setup, each crate is stocked with 10,000 5-day-old-larvae (5-DOL) and $3 \mathrm{~kg}$ of shredded biowaste. Additional feeding at day four $(5 \mathrm{~kg})$ and day seven $(4 \mathrm{~kg})$ amounts to the total of $12 \mathrm{~kg}$ treated over a period of 12 days. It was observed that the larvae between the age of five and nine days do not require the full surface area in the crate. Based on this observation a trial was designed to test if the spatial footprint of the conversion units and its handling time can be reduced by reducing the crate size in the early age of the larvae without negatively affecting the performance and low-tech nature of the BSF system developed. For the first part of the 12 -day feeding period, smaller incubator containers $(30 \times 20 \times 10$ $\mathrm{cm}$ ) were used to each accommodate 10,000 5-DOL and fed either 1 or $1.5 \mathrm{~kg}$ as a first feeding. Different setups then tested an emptying of the incubator container into the standard conversion crate after 2, 3 or 4 days. Thereafter a varied amount of waste was fed, in one or two additional feedings. A trial using the standard feeding regime and crate sizes was used as control. All thirteen trials fed a total of $12 \mathrm{~kg}$ of waste to 10,000 larvae over a duration of 12 days. Each feeding regime was setup in triplet and larval mass was measured on day 0,7 and 12. Parameters measured on day 12 were survival rate, waste reduction and biomass conversion rate (BCR). Comparing the results between the thirteen trials, a high larval mass, survival rate and BCR in the setup where larvae were fed 1 and $1.5 \mathrm{~kg}$ of biowaste in incubator containers in combination with one feeding of 11 and $10.5 \mathrm{~kg}$ in the conversion crates after three days was found. Such a setup for biowaste treatment would reduce the spatial footprint of the conversion system by around $20 \%$. Handling time is also reduced as the conversion units do not need to be dismantled for a third feeding which is the case with the current setup. 


\title{
Synchronous front-face fluorescence measurement as a method to rapid discrimination of edible insect powders
}

\author{
G. Rossi", S. Bußler, J. Durek, S. Ojha and O. Schlüter \\ Quality and Safety of Food and Feed, Leibniz Institute for Agricultural Engineering and Bioeconomy (ATB), Potsdam, \\ Germany; grossi@atb-potsdam.de
}

Edible insects are being suggested as a future food and feed source. To promote their widespread on a large scale, comprehensive research studies are required. Identification and classification of insects in the context of knowing their origin and characterising the technological treatments during processing could support the consumer acceptance. Rapid and non-destructive methods are therefore needed for quality control purpose. Fluorescence spectroscopy is a powerful tool to perform various analyses on environmental and food matrices and could also play an important role in in the context mentioned above. Total synchronous fluorescence spectroscopy, a technique that measures the emission intensity by moving along excitation and emission wavelengths in sync, was applied at several wavelengths shifts $(\Delta \lambda)$ in order to classify edible insect powders. For this purpose, seven different insect species (Acheta domesticus, Gryllus bimaculatus, Gryllus assimilis, Locusta migratoria, Schistocerca gregaria, Tenebrio molitor, Zophobas morio) were freeze-dried and ground to record synchronous fluorescence spectra between 250 and $600 \mathrm{~nm}$ with $\Delta \lambda$ ranged between 10 and $120 \mathrm{~nm}$ in $10 \mathrm{~nm}$ steps. Recorded data revealed clear differences in the emission spectra of powder from different insect species. Principal components analysis (PCA), linear discriminant analysis (LDA) and partial least-square discriminant analysis (PLS-DA), were applied on the whole set of data in order to investigate the ability of specific detected fluorescence signals to classify insect powders. Therefore, single $\Delta \lambda s$ were separately studied. PCA, LDA and PLS-DA applied on each set of data allowed to obtain good classification and prediction. Results of the current analysis suggest that synchronous fluorescence coupled with powerful chemometric methods could offer a promising tool to identify the composition of different mixed fractions of intermediate insect powders.

\section{Developing of low-impact diets for the mass rearing of Acheta domesticus}

\author{
C. Jucker ${ }^{*}$, S. Belluco ${ }^{2}$, D. Lupi ${ }^{1}$, S. Savoldelli ${ }^{1}$, L. Bonizzi ${ }^{3}$, A. Ricci ${ }^{2}$, A. Mascaretti ${ }^{3}$ and L. Gasco ${ }^{4}$ \\ ${ }^{1}$ Department of Food, Environmental and Nutritional Sciences, University of Milan, via Celoria 2, 20133 Milan, Italy; \\ ${ }^{2}$ Istituto Zooprofilattico Sperimentale delle Venezie, Viale dell'Università 10, 35020 Legnaro (PD), Italy ${ }^{3}$ Department of \\ Biomedical, Surgical and Dental Sciences, University of Milan, via della Commenda 10, 20122 Milano, Italy; ${ }^{4}$ Centro per lo \\ Sviluppo sostenibile, via Daverio 7, 20122 Milan, Italy; ${ }^{5}$ Dipartimento di Scienze Agrarie, Forestali e Alimentari, Unversità \\ degli Studi di Torino, L. go Braccini 2, 10095 Grugliasco (TO), Italy; costanza.jucker@unimi.it
}

In the recent framework of insect as sustainable protein source for humans, Acheta domesticus, the house cricket, is one of the species of major interest due to its high protein content. This species is reared worldwide and consumed whole or as a meal. Its mass rearing relies on grain feeds, using ingredients in competition with humans and animals, and with a high cost. By-products represent a useful source of feed for insects as they are available in huge quantity, low cost, often have a good nutritional profile and represent a waste for humans. Thus, they contribute to lower the environmental impact of insect production and its cost, that is negatively influenced by the cost of the rearing substrate. Furthermore, the use of waste as growing media contribute to its management and valorisation. In order to identify an optimal feed formulation for crickets, respecting the low environmental costs, in this study (included in the Project MAIC - model for rearing edible insects), we tested different diets formulated with low-cost raw-materials and by-products. Different ingredients were chosen considering the nutritional composition, the cost and the availability on local agro-food industries. Four diets were formulated using different percentage of the selected raw-material and by-products and with different levels of crude proteins, lipids and nitrogen-free extracts. Following by-products were used: brewery waste (trub or brewery' spent grain), maize distiller, rice hulls and soybean hulls. Experimental diets and the control (chicken feed) were provided in triplicates to a group of neonate A. domesticus reared at the University of Milan. Different growth parameters, the survival and the efficiency of the conversion of the ingested food were assessed until adult stage was reached. Though a high mortality was observed in all the tested diets influencing the final results, two diets with a protein content between 12 and $16 \%$ were identified as more promising and have to be deepen with supplemental trials. This study adds some useful information to the few data available on the identification of an optimal diet, which includes by-products or food waste, for the mass rearing of the house cricket. 


\title{
Lost in the desert: influence on wet feed distribution on the density and growth rate of Tenebrio molitor
}

\author{
D. Deruytter", C.L. Coudron, J. Claeys and S. Teerlinck \\ Inagro, Ieperseweg 87, 8800 Rumbeke-Beitem, Belgium; david.deruytter@inagro.be
}

To efficiently rear insects, it is important to use a feed that provides an optimal growth and is distributed homogeneously. One of the challenges in rearing mealworms is the fact that they need both a dry feed (e.g. wheat bran) and a wet feed (e.g. carrots). The dry feed is not a problem as it is a homogeneous mixture that is easily applied evenly in the crate by conventional techniques. In contrast, wet feed may result in some problems. In many small-scale rearing facilities, its given daily by hand, but this does not necessarily result in an even distribution of the wet feed. But even automated systems may struggle to evenly distribute wet feed. Common sense and experience taught us that if the (wet)feed is not distributed evenly, mealworm spatial distribution is not even, and the growth is not optimal. With this experiment we want to quantify the distance to the wet feed before adverse effects start to occur (on density, growth rate and growth rate variability). This was done by placing mealworms in a $60 \times 40 \mathrm{~cm}$ crate with ad libitum supply of wet feed (agar) on one side. After 7 days, the distribution, average and individual weight of the mealworms was assessed in 7 zones depending on the distance to the wet feed $(0,2.5,5,10,15$, $25,40,60 \mathrm{~cm}$ ). This was done for 8 weight classes (between 0.7 and $125 \mathrm{mg}$ ) and 4 replicates. As expected, the results indicate that size matters. The smallest assessed size $(0.7 \mathrm{mg})$ seemed too small to move through the dry feed resulting in a limited influence on the spatial distribution. However, the growth was adversely affected when the wet feed was further than $5 \mathrm{~cm}$ away. The strongest influence was observed for mealworms with an initial weight between 3 and $35 \mathrm{mg}$. It appeared that this category was mobile enough to crawl to the wet feed but not mobile enough to redistribute afterwards. This results in a densely populated 'active zone' with fast and evenly growing mealworms and a virtual 'dead zone' further than 10 to $20 \mathrm{~cm}$ from the wet feed (depending on the size). In this dead zone growth was up to $50 \%$ lower and more variable with a density of only $10 \%$ compared to the active zone. Above an average initial weight of $35 \mathrm{mg}$, the distance to the wet feed does not seem to matter for the growth and influence on the density is low. In conclusion, homogenous distribution of the wet feed is important, certainly for mealworms below $35 \mathrm{mg}$. If not, this results in a skewed mealworm distribution, in a skewed dry feed availability, a lower growth rate and increase the overall size variability. A rule of thumb that can be used is to multiply the average weight $(\mathrm{mg})$ by 4 and this is the maximum distance between two sources of wet feed if you do not want adverse effects on the growth.

\section{My way or the highway: transport conditions for Tenebrio molitor}

\author{
J. Claeys", C.L. Coudron, D. Deruytter and S. Teerlinck \\ Inagro, Ieperseweg 87, 8800 Rumbeke-Beitem, Belgium; jonas.claeys@inagro.be
}

As insect rearing is becoming more and more specialised, it is to be expected that, analogous to the breeder-broiler distinction in poultry, a homologous distinction will come to be in insect breeding. Companies dedicated to reproducing insects will supply dedicated rearing companies with young livestock. In this regard, transportation of different life stages of the insect is becoming more and more a common practice. In optimal conditions, with minimal interference for the insect, transportation is short and happens under controlled atmosphere, however these specific requirements will have an impact on the price of transportation. Financially, it is of most interest to have no specific transportation requirements, but prolonged exposure of the insects to suboptimal conditions during transportation will have an influence on survivability and might interfere with the reproductivity after maturation of the insects. What is the cost-efficiency-optimum for transporting insects (e.g. the same parcel in Belgium without requirements will cost $€ 3.90$, while an express transport will cost up to $€$ 161.7). One must consider that this optimum will differ between insect species, but also between insect life stages and even more within the same life stage of an insect specie depending on the purpose of this insect, will it be used for production and harvest only or will it serve for reproduction later in its life cycle. This research focuses on mealworm (Tenebrio molitor), initially on the egg and young larval stage. A literature study was performed and research already suggested that the duration of the exposure also affects the outer margins of the viable climate conditions, while a combination of extreme conditions (low/high temperature at low/high relative humidity) are detrimental for prolonged quality. To simulate transportation conditions, eggs and young mealworm are exposed to different combinations of temperature $\left(5-30{ }^{\circ} \mathrm{C}\right)$ and relative humidity $(\mathrm{RH})(30-90 \%)$ in a phytotron for a varying amount of time (1-6 days). After exposure to the transportation conditions, the mealworm are further reared at $27^{\circ} \mathrm{C}$ at $60 \%$ and survival is monitored. These experiments give an indication of the acceptable ranges for the transportation conditions and the need for customised transport conditions. 


\title{
Exploring feed preferences of black soldier fly larvae
}

\author{
A. Parodi ${ }^{1}{ }^{*}$, K. van Dijk ${ }^{1}$, J.J.A. van Loon ${ }^{2}$, I.J.M. de Boer ${ }^{1}$, J. van Schelt ${ }^{3}$ and H.H.E. van Zanten ${ }^{1}$ \\ ${ }^{1}$ Animal Production Systems group, Wageningen University E Research, P.O. Box 338, 6700 AH Wageningen, the Netherlands; \\ ${ }^{2}$ Laboratory of Entomology, Wageningen University E Research, P.O. Box 16, 6700 AA Wageningen, the Netherlands; ${ }^{3}$ Bestico, \\ Veilingweg 6, 2651 BE Berkel en Rodenrijs, the Netherlands; alejandro.parodiparodi@wur.nl
}

The attention for black soldier fly larvae (BSFL) as an alternative ingredient for food and feed products is on the rise. While many studies have reported the efficiency of BSFL to bio-convert a wide range of organic waste streams into larval biomass, so far, it is unknown if BSFL prefer certain waste streams over others when they have the possibility to choose. Here we performed a choice-test experiment to explore the preference of BSFL when exposed to pig manure and a mass-rearing diet consisting of plant by-products currently used for industrial BSFL production. We found that after 1-hour of exposure to both feeds, BSFL strongly preferred pig manure over the mass-rearing diet at different ages. Our results provide the first evidence that BSFL express a distinct diet preference. Understanding the reasons for the strong preference for manure is relevant for a diverse array of practical applications and to inform the discussion on insect welfare.

\section{Effect of prepupae density in pupation crates on emergence rate of black soldier flies}

T. Rahayu, T. Kusumawardhani, B.M.A. Dortmans" and C. Zurbrügg

Eawag - Swiss Federal Institute of Aquatic Science and Technology, Department of Sanitation, Water and Solid Waste for Development (Sandec), 8600 Dübendorf, Switzerland; bram.dortmans@eawag.ch

Increasing efficiency in a black soldier fly (BSF) rearing unit, entails reducing the required space footprint and labour without compromising the performance. At the BSF rearing unit, as implemented by the FORWARD project in Sidoarjo, Indonesia, the longest retention time in the rearing process is in the pupation unit where prepupae enter the pupation stage, pupate and then emerge as flies. A previous trial tested the possibility to increase densities of prepupae in $61 \times 43 \times 10 \mathrm{~cm}$ pupation crates (using densities of 2,500-7,500 prepupae per crate) and investigated the effect on the emergence rate of the flies. The results revealed that with the densities tested no effect on the emergence rate could be found and it was concluded that densities could be further increased. For the study presented here, 6 different densities between 7,500 and 20,000 prepupae per crate were tested and evaluated. The prepupae used for this trial were fed as larvae on a homogenised and portioned diet of fruit waste and chicken feed (87.5 and 12.5\% respectively). Each dark cage (pupation cage) was filled with 16 crates containing prepupae of one density group. Prepupae weight varied between $113 \pm 4.7$ and $164 \pm 20.7 \mathrm{mg}$. Prepupae were added to all crates of each density group over a period of 5 days and crates were added to dark cages after eight days. The emerged flies were harvested daily over a period of two weeks by connecting the pupation cage to one or more oviposition cages for 1 hour per day per cage using a light source to enhance migration of the emerged flies. Total number of flies harvested from each density group was calculated based on the average weight of a triplet sample of 20 flies emerged from each dark cage. The emergence rate is calculated based on the number of prepupae per pupation cage and the number of harvested flies. Further parameters measured include a fly mortality analysis in each dark cage and ambient temperature combined with relative humidity measured in the BSF nursery room every 10 minutes over the complete duration of the trial. Preliminary results show that with the tested range a maximum prepupae density can be determined. Beyond that density, a decrease in emergence rate can be observed. The tested range could increase performance of the rearing unit by nearly threefold per square meter compared to the current prepupae density, impacting significantly on the space footprint and labour requirement. 


\title{
Optimisation of treatment duration in black soldier fly larvae composting
}

\author{
E. Ermolaev", C. Lalander and B. Vinnerås \\ Swedish University of Agricultural Sciences (SLU), Department of Energy and Technology, P.O. Box 7032, 75007 Uppsala, \\ Sweden; evgheni.ermolaev@slu.se
}

In large-scale implementations of fly larvae composting, the shorter treatment duration allows using the treatment surface more efficiently and has potential of reducing ventilation and heating costs per ton of treated waste. This study investigated the possibility of development of feed demand model in order to estimate the optimal feed amounts and times to achieve a shorter treatment time without reduction in conversion efficiency. The modelling should take into consideration two major aspects of black soldier fly larvae composting of food waste: larval feed demands associated with their growth and water removal. These two aspects were evaluated at different scales using 5-day-old BSF larvae sourced from the continuously running BSF colony at SLU. Experiments in small scale were performed in boxes with pig feed as substrate and with a range of larvae densities $\left(0.6-2 \mathrm{lv} / \mathrm{cm}^{2}\right)$ and treatment durations (4-14 d) based on projection of achieving a similar bioconversion ratio (25\%) at the end of the treatment. Experiments at the large scale were performed in standard treatment crates with a surface of $2,400 \mathrm{~cm}^{2}$ with minced food waste as substrate and a range of larvae densities $\left(5-17 \mathrm{lv} / \mathrm{cm}^{2}\right)$ and treatment durations (4-14 d) based on the feed demand model evaluated in the small-scale experiments. The results showed that in small-scale experiments, the actual feed demand followed closely the predicted values, making it possible to design the treatment to obtain an optimal bioconversion ratio with a treatment time as short as 4 days, or alternatively to obtain a desired size of larvae within a shortest treatment time possible. The large-scale experiments showed that while the process followed the feed demand model, too high moisture in the food waste prevented the treatment achieving the designed bioconversion within the projected treatment time. Instead the process had to continue for roughly the same time in order to accommodate the water removal from the substrate. Indeed, the water removal model recently developed in our research group suggests that water removal rate from the fly larvae composting process largely depends on the initial substrate properties and the ventilation capacity of the treatment room. Applying these recent findings to the current study suggests, that the large-scale implementation of the feed demand model should always be adjusted to the duration required for water removal and the system should be optimised for achieving the optimal bioconversion ratio within this period.

\section{Comparison of black soldier fly eclosion success in two environments: the impact of variance in temperature and humidity}

\author{
V. Wiklicky" and C. Lalander \\ Department of Energy and Technology, Swedish University of Agricultural Sciences, P.O. Box 7032, 75007 Uppsala, Sweden; \\ viktoria.wiklicky@slu.se
}

Mass rearing the black soldier fly (Hermetia illucens) (BSF) has increased in recent years as BSF is emerging as a novel farmed animal for industrial purposes. In literature, BSF farming has been primarily reported in either a laboratory or a greenhouse $(\mathrm{GH})$ setting, which contrast in the level of environmental control. Both settings can maintain a viable colony, but no study has yet compared the productivity between the two types of environments. It could be assumed that, depending on the setting, variability in temperature $(\mathrm{T})$ and relative humidity $(\mathrm{RH})$ would lead to different rearing success of BSF. Particularly in a GH environment, where the parameters under which BSF are farmed can vary depending on the geographical location, e.g. hours of daylight, and seasonal conditions. Knowing the impact of the variability of environmental parameters on the production efficiency helps to gain better control over the BSF rearing process and improvements could increase the efficiency. In this ongoing study we evaluate the impact of variation in $\mathrm{T}$ and $\mathrm{RH}$ on egg eclosion rates, by comparing a setting with high variation to one with low variation. The first setting is a heated GH in a cold climate, in which the average year-round indoor temperature is $28.5 \pm 3.5^{\circ} \mathrm{C}$ and relative humidity is $45 \pm 15 \%$. The GH is connected to the outside environment through insect-proof flaps and a sprinkler emits fine mist every 4 minutes. The variance in $\mathrm{RH}$ in the GH setting is caused by $\mathrm{RH}$ fluctuation with the outside conditions and it is expected to be higher with lower temperatures and vice versa. The second setting is a highly regulated climate chamber. The eclosion rate was evaluated for five environmental conditions resembling the conditions in the GH setting: (1) $25^{\circ} \mathrm{C} / 30 \% \mathrm{RH}$; (2) $25^{\circ} \mathrm{C} / 60 \% \mathrm{RH}$; (3) $32{ }^{\circ} \mathrm{C} / 30 \% \mathrm{RH}$; (4) $32{ }^{\circ} \mathrm{C} / 60 \% \mathrm{RH}$; (5) $28{ }^{\circ} \mathrm{C} / 45 \%$ $\mathrm{RH}$. In the GH setting, experiments were run over a period of several weeks throughout the year, whereas in the controlled setting, triplicates were run for 5 days for each condition. One gram of BSF eggs were placed on standardised feed material and after 5 days, the number of estimated hatchlings were compared to the actual number of hatchings and expressed as hatching success (\%). Preliminary results suggest that settings to enhance conditions, such as T and RH, can optimise the rearing efficiency of the BSF rearing process. Adjustments of the colony with the here suggested adjustments can lead to an increased eclosion which is interesting for low-tech farms how to potentially improve their rearing efficiencies with small adjustments in existing settings or help to guide the type of setup for those planning to establish new farms. 


\title{
New vertical solution in automatic black soldier fly production
}

\section{B. Holtermans}

Insect Engineers B.V., Nijverheidsstraat 2a, 5961 PJ Horst, the Netherlands; info@insectengineers.com

In the development of black soldier fly (BSF) farming a lot of focus has been on breeding and processing. For logic and practical reasons. Because breeding is the start of your process and processing is the last step in your process. In addition, this could all be started and tested on small scale. The middle step, production, was often not looked at. Currently the market is that far developed that there are proper solutions for breeding and processing. But large-scale production on an economical and efficient way seems to be difficult. Our focus has been on that step. To realise this, we stepped away from the traditional trays and we focused on vertical farming. For the simple reason that we have experience in vertical farming because of our background in the mushroom industry. These vertical racks can vary in height and length. A good standard would be 8 levels high and $30 \mathrm{~m}$ long vertical racks. The racks are standing in a climate-controlled production room. Important change in comparison to tray systems is that we don't bring the BSF to the machinery, but we bring the machinery to the BSF. On this way we can keep the conditions for the BSF in the most optimal condition because they stay in the controlled growing room. You lose all the transport movements which also means the technology and effort to realise that. Focus is on not having moving parts in the production room. The machines are mobile which makes it simple and cost effective to scale up the whole production. Filling, multiple feed and emptying of the vertical racks is an automated system. Three persons are enough to do this operation. Big difference with some Western solutions are that you need workers to operate this equipment and not professors. So, this system can be supplied worldwide, from Kenya to Canada. It's low in investment per $\mathrm{m}^{2}$ and seems to be the most idiot proof production solution on the market. Latest development is an update on the climate system. At point of writing this submission it's too early to tell. What we can say is that we see more than double the results when it comes to effectively densify the larvae production because of this new climate system.

\section{Optimising black soldier fly breeding in tropical conditions}

\author{
A.S. Malawey", A.M. Roslan and M.J. Zorrilla \\ Nutrition Technologies Sdn Bhd, Lot PLO 2 \& 3 Kawasan Perindustrian, 81400, SenaiLot PLO 2 E 3 Kawasan Perindustrian, \\ 81400, Johor, Malaysia aline@nutrition-technologies.com
}

The research conducted in the last 20 years has shown that black soldier fly larvae are a reliable source of protein and lipid in feeds for farmed animals, especially for aquaculture. Breeding these flies under optimal and stable abiotic parameters often requires indoor facilities. Those are highly costly, require a large amount of empty space, and most of the time their egg production does not match that of outdoor cages. However, in places with plenty of sunlight and stable abiotic parameters, optimal outdoor cage designs can bring the costs of mass production to significantly lower levels than indoor facilities. Here, we present some details of the past and current outdoor insectarium designs developed by Nutrition Technologies that can optimise the mass production of this insect to meet demands, at a low cost and reduced environmental footprint. An Overview of the cage designs and key lessons learned will be shared. 


\title{
Gaseous emissions during black soldier fly rearing in a mass-rearing diet and pig manure
}

\author{
A. Parodi ${ }^{1 *}$, I.J.M. de Boer1, W.J.J. Gerrits ${ }^{2}$, J.J.A. van Loon ${ }^{3}$, M.J.W. Heetkamp ${ }^{4}$, J. van Schelt ${ }^{5}$, J.E. Bolhuis ${ }^{4}$ and $^{1}$ \\ H.H.E. van Zanten ${ }^{1}$ \\ ${ }^{1}$ Animal Production Systems group, Wageningen University E Research, P.O. Box 338, 6700 AH Wageningen, the Netherlands; \\ ${ }^{2}$ Animal Nutrition group, Wageningen University E Research, P.O. Box 338, 6700 AH Wageningen, the Netherlands; \\ ${ }^{3}$ Laboratory of Entomology, Wageningen University E Research, P.O. Box 16, 6700 AA Wageningen, the Netherlands; \\ ${ }^{4}$ Adaptation Physiology group, Wageningen University E Research, P.O. Box 338, 6700 AH Wageningen, the Netherlands; \\ ${ }^{5}$ Bestico BV, Veilingweg 6, 2651 BE Berkel en Rodenrijs, the Netherlands; alejandro.parodiparodi@wur.nl
}

Black soldier fly larvae (BSFL) are increasingly seen as ideal candidates to turn organic streams into biomass that could be upcycled in the food system as either animal feed or human food. While many studies have focused on finding nutrient conversion efficiencies of BSFL fed on a wide variety of organic streams, limited attention has been given to the gaseous emissions produced during the rearing. The quantification of these gaseous emissions is crucial to understand emission patterns, develop mitigation strategies, and clarify the potential environmental benefits of utilising them as feed or food. Here, we reared 7-days old BSFL larvae in respiration chambers to continuously measure the production of gases of environmental concern (i.e. $\mathrm{CO}_{2}, \mathrm{CH}_{4}, \mathrm{~N}_{2} \mathrm{O}$ and $\mathrm{NH}_{3}$ ). Two different substrates were tested: a mass rearing diet made from by-products of plant origin, and pig manure. With the mass rearing diet, nearly no $\mathrm{CH}_{4}$ was detected. $\mathrm{CO}_{2}$ and $\mathrm{N}_{2}$ emissions quickly increased up to the $5^{\text {th }}$ day of measurements and then dropped. By contrast, $\mathrm{NH}_{3}$ was only produced after the peak in $\mathrm{CO}_{2}$ levels occurred. We found evidence that more than $30 \%$ of the $\mathrm{CO}_{2}$ produced was produced by the microbial biomass of the substrate. When BSFL was reared in pig manure, methane was produced during the first 3 days of measurements and then steadily dropped. $\mathrm{CO}_{2}$ and $\mathrm{NH}_{3}$ were produced all along but reached their maximum during the $6^{\text {th }}$ and $7^{\text {th }}$ day. $\mathrm{N}_{2} \mathrm{O}$ steadily increased over time. In addition, we found that when BSFL were reared in pig manure, $\mathrm{NH}_{3}$ emissions were twice higher than those found for pig manure without BSFL. Our results are relevant for the improvement of BSFL conversion efficiencies and for the reduction of gaseous emissions during BSFL rearing. 


\section{Biotechnology \& non-food applications of insect}

\section{Effects of black soldier fly frass-fertiliser on the growth, yield and nitrogen use efficiency of maize in Central Kenya}

D. Beesigamukama ${ }^{1,2,3^{*}, \text { B. Mochoge }}{ }^{2}$, N.K. Korir ${ }^{2}$, D. Nakimbugwe ${ }^{4}$, K.K.M. Fiaboe ${ }^{1,5}$, F.M. Khamis ${ }^{1}$, S. Subramanian $^{1}$, M.W. Musyoka ${ }^{1}$, S. Ekesi ${ }^{1}$ and C.M. Tanga ${ }^{1}$

${ }^{1}$ International Centre of Insect Physiology and Ecology P. O. Box 30772-00100, Nairobi, Kenya; ${ }^{2}$ Department of Agricultural Science and Technology, Kenyatta University P.O. Box 43844-00100, Nairobi, Kenya; ${ }^{3}$ Department of Crop Production and Management, Busitema University P.O. Box 236 Tororo, Uganda; ${ }^{4}$ Department of Food Technology and Nutrition, School of Food Technology, Nutrition E Bioengineering, Makerere University, P.O. Box 7062, Kampala, Uganda; ${ }^{5}$ The International Institute of Tropical Agriculture (IITA), B.P. 2008 (Messa), Nkolbisson, Yaoundé, Cameroon; dbeesigamukama@icipe.org

The black soldier fly larvae (BSF) (Hermetia illucens L.) is one of the most efficient recyclers of organic waste into quality organic fertiliser. However, no information exists on the performance of BSF frass-fertiliser on crop production. Such information is crucial for the development of commercial BSF frass-fertiliser and its integration into existing farming systems. We carried out comparative studies to evaluate the impact of BSF frass-fertiliser and commercial organic fertiliser (SAFI) applied in terms of tonnage $[0,2.5,5$ and 7.5 tonnes per hectare $(\mathrm{t} / \mathrm{ha})]$ and nitrogen content $[0,30,60$ and $100 \mathrm{~kg}$ nitrogen $(\mathrm{N})$ per hectare ( $\mathrm{kg} \mathrm{N} / \mathrm{ha})$ ] on maize (H513 variety) production under open field conditions. A standard mineral fertiliser treatment (urea) [0, 30, 60 and $100 \mathrm{~kg}$ nitrogen $(\mathrm{N})$ per hectare $(\mathrm{kg} \mathrm{N} / \mathrm{ha})]$ was also included. The performance of H513 maize was assessed based on the chlorophyll content, $\mathrm{N}$ uptake, grain yield and $\mathrm{N}$ use efficiency. Our results showed that application of BSF frass-fertilisers significantly increased maize grain yields compared to the other fertilisers. Plots treated with $100 \mathrm{~kg} N / \mathrm{ha}$ BSF frass-fertiliser produced the highest maize grain yield of $5.7 \mathrm{t} / \mathrm{ha}$, which was $6 \%$ higher compared to that produced by equivalent rates of SAFI and Urea fertilisers. Furthermore, the application of BSF frass-fertiliser at $7.5 \mathrm{t} /$ ha significantly increased the chlorophyll contents of H513 maize by 4 and 9\% compared to SAFI and Urea fertilisers, respectively. The application of BSF frass-fertiliser at $100 \mathrm{~kg} \mathrm{~N} /$ ha also increased the N uptake by 39\% compared to SAFI and 21\% in relation to Urea treated plots. Maize agronomic N use efficiency in BSF frass-fertiliser treated plots was 27 and 116\% higher compared to SAFI and Urea treated plots, respectively. Our findings from the study highlight that the use of appropriate BSF frass-fertiliser in maize production could optimise the response and utilisation efficiency of applied N, which is the most limiting nutrient to crop production in the tropics. 


\title{
Effect of composted black soldier fly larvae frass on plant growth performance
}

\author{
M.J. Zorrilla" and Z.B.M. Hussain \\ Nutrition Technologies Sdn Bhd, Lot PLO 2 \& 3 Kawasan Perindustrian, 81400 Senai, Johor Malaysia; \\ martin@nutrition-technologies.com
}

When black soldier fly larvae (Hermetia illucens) are reared for animal feed, the raw material produced in greatest volume is not the protein or oil but the frass. Frass is considered a soil amendment, however its commercial applications and the benefits of composting the product have not been thoroughly studied. Through several field trials, Nutrition Technologies has examined the effect of an 'unprocessed frass' and an 'aerobically composted frass' on plant growth performance. The frass products were compared to soil amendments and fertilisers commercially available in Southeast Asia. The composted frass products resulted in improved growth performance when compared to un-processed frass. Composted frass outperformed commercially available soil amendments, while results for un-processed frass were more varied. Detailed results from several field trials involving several commercially important crops will be shared.

\section{Progression towards circular agriculture - gaining knowledge of insect waste streams as a crop and soil health promoter}

\author{
K.L. Torgerson ${ }^{1 *}$, J.V. Meijering ${ }^{2}$, J. Sok ${ }^{1}$, M. Dicke ${ }^{2}$ and A.G.J.M. Oude Lansink ${ }^{1}$ \\ ${ }^{1}$ Wageningen University \& Research, Hollandseweg 1, 6706 KN Wageningen, the Netherlands; ${ }^{2}$ Wageningen University \& \\ Research, Droevendaalsesteeg 1, 6708 PB Wageningen, the Netherlands; kirstin.torgerson@wur.nl
}

Insect production is gaining traction as a form of mini-livestock production. As the sector grows, more insect waste streams (IWS) (i.e. insects' manure, moulted exoskeleton, and undigested feed) accumulate. Preliminary research suggests that IWS have the potential to promote the health of crops and soil that induce a plant's systemic resistance to pathogens and pests. In doing so, IWS could reduce, for instance, arable farmers' usage of chemical plant protection products (e.g. pesticides such as neonicotinoids) thereby reducing their environmental consequences. Also, the use of IWS as an arable farming input aligns with the vision of circular agriculture by progressing towards closing the loop of insect production. To successfully penetrate the market as an arable farming input, farmers must first be informed of how IWS are expected to work in practice. However, such information is unknown; the objective of this research is to supply such needed information. This paper explores the Dutch farming context as the Netherlands is a driver of insect production R\&D and hosts several large production facilities. Experts with knowledge of IWS and its potential application on Dutch arable farms participated in this research to determine (1) what IWS are, (2) how IWS promote the health of plants and soil, and (3) how IWS should be used in practice. Such elicited information is in accordance with Rogers' theory regarding the knowledge required by one to progress through the innovation decision process. This research used a combination of semi-structured interviews and a 3-round Delphi study. Interviews were conducted to generate individual understandings of IWS. The results from the interviews were compared, and areas of divergence in expert opinion were identified. These areas of diverging opinion were then presented to experts in a Delphi study to determine to what extent they were able to reach an agreement. Results from the interviews were analysed qualitatively; the Delphi study was analysed quantitatively to calculate the Strict Agreement Index. This expert elicitation yields new insights regarding the technical mechanisms and potential applications of IWS which were previously unknown due to IWS being in the R\&D phase. Such results provide a launching pad for further research in the adoption potential of IWS. The results also identify critically unanswered questions that simply cannot be addressed until more technical research on IWS' effectiveness is performed. 


\title{
Fate of organic waste and pesticides in black soldier fly compositing
}

\section{Kipkoech ${ }^{{ }^{*}}$ and C.M. Tanga ${ }^{2}$}

${ }^{1}$ Jomo Kenyatta University of Agriculture and Technology, Department of Food Science and Technology, P.O Box 6200000200, Nairobi, Kenya; ${ }^{2}$ International Centre of Insect Physiology and Ecology (icipe), P.O. Box 30772-00100, Nairobi, Kenya; kipkoechcarolyne@gmail.com

Organic waste management strategy is gaining great attention as a means of compacting environmental degradation. Uncontrolled use of pesticides, especially in sub-Saharan Africa, has caused increased pesticide residue in the environments and surrounding water bodies. Black soldier fly larvae is gaining increased consciousness in Kenya, and other countries in the world due to its enormous composting ability. This study evaluates the fate of 15 commonly used agricultural chemicals in Kenya in larvae composting system and in control without larvae. The results would inform stakeholders to impede the spread of agricultural chemicals waste into the environment hence improve environmental remediation.

\section{In vitro antimicrobial activity of frass produced by the black soldier fly larvae against plant pathogens}

\author{
G. Arabzadeh $^{1 \#}$, T.T.A. Nguyen ${ }^{2 \#}$, M. Delisle-Houde ${ }^{2}$, M. Dorais ${ }^{2}$, M.H. Deschamps ${ }^{1}$, N. Derôme ${ }^{3}$, R.J. Tweddell ${ }^{2}$ \\ and G.W. Vandenberg ${ }^{1^{*}}$ \\ ${ }^{1}$ Département des sciences animales, Université Laval, Québec (Québec), G1V 0A6, Canada; ${ }^{2}$ Département de phytologie, \\ Université Laval, Québec (Québec), G1V0A6, Canada; ${ }^{3}$ Département de biologie, Université Laval, Québec (Québec), G1V \\ 0A6, Canada; grant.vandenberg@fsaa.ulaval.ca; "these authors contributed equally
}

In 2020, the control of plant diseases still relies on the use of pesticides. Negative impacts of these chemical compounds on environment and human health are now demonstrated and research for new low risk alternatives is essential. Over the past few years, it was possible to note a marked interest for the cultivation of insects as well as for the various by-products arising therefrom. In this context, an organic residue called 'frass' produced by the black soldier fly larvae [Hermetia illucens (Linnaeus, 1758)] was evaluated for its antimicrobial activity. The aim of this study was: (1) to determine in vitro antifungal activity according to different diets (Gainesville 100\%, Gainesville:VOWaste 70:30, Gainesville:VOWaste 50:50); and (2) to look for the presence of antibacterial compounds in frass solutions. Antimicrobial activity was evaluated using tryptic soy agar (TSA) medium by the double layer test for fungi and agar disc diffusion test for bacteria. For fungi, inhibition of mycelial growth (\%) was determine according to reduction of mycelial diameter on TSA containing frass (0.1\%) in comparison with the control (distilled sterile water). For bacteria, inhibition of bacterial growth was determined on semi-solid TSA medium by measuring the diameter of inhibition zone surrounding discs soaked with different concentrations of frass solutions previously sterilised with cold microfiltration at 0.2 micron. In both tests, horticultural plant pathogens were used. The results showed the high antifungal activity of frass solutions. Frass from larvae colonies using Gainesville $100 \%$ as a diet showed inhibition of mycelial growth $>90 \%$ against all fungi tested. Both other diets tested showed inhibition growth between 30 and $85 \%$. It would now be interesting to evaluate the possibility of spray frass solutions on horticultural crops to control plant diseases. 


\title{
Search for antimicrobial molecules from lactic acid fermentation of black soldier fly prepupae and related byproducts
}

\author{
A.V. Luparelli", J. Hadj Saadoun, A. Ricci, E. Neviani, G. Galaverna, C. Lazzi, A. Caligiani and S. Sforza \\ Department of Food Science and Drug, University of Parma, Parma, Italy; annavalentina.luparelli@unipr.it
}

The steadily growth of world's population leads to the continuous search for new strategies to reduce and add value to organic wastes generates by the increased global agriculture production. For this purpose, a non-infesting insect, Hermetia illucens, is often used in the bio-conversion processes. The growth of this specie of insect on organic waste is a sustainable way to upgrade the nutritional value of the bio-residues. During this process, large amount of insect-derived wastes (chitinous shells and dead adult flies) and excess of larvae are also accumulated. Unlike the valorised biomass, which has a lot of applications, there is still not a sustainable way of using the insects derived wastes and the residual larvae. Recent studies are marking how the use of insects as feed are giving positive effects on the pathogen's growth. At the same time, already known in literature is that fermentation help the production of antimicrobial compounds. In this work these two evidences were combined for exploring antimicrobial potential from insects' wastes, using solid state fermentation with Lactic Acid Bacteria. The difference in the molecular composition between fermented and unfermented insect-derived waste materials was studied, focusing on fatty acid profiles, protein fraction and chitin. Solid state fermentation was conducted with 2 lactic acid bacteria strains Lactobacillus rhamnosus and Lactobacillus plantarum. Both strains had shown the highest antimicrobial activity, tested against foodborne pathogens, when they were grown on puparium. The bulk evaluation of fat, protein, moisture and ashes composition of insect-derived biomasses was carried out using standard procedures. Then, fatty acid profiles were determined by GC-MS, whereas total amino acid quantification and total amount of chitin were simultaneously carried out by UPLC/ESI-MS methodology. The high antimicrobial activity against foodborne pathogens of the fermented substrates compared to unfermented, with a reduction of $5 \log$ UFC/g, suggest the formation of molecular components responsible for the antimicrobial activity. Results showed that the lipid fraction is particularly influenced by fermentation: the fermented biomass had a lower percentage of Lauric acid and Meristic acid and a higher quantity of palmitic acid and C:18 series. Also, the protein fraction seemed to change upon fermentation: it was possible to observe a redistribution of amino acids with differences related especially to different growth substrate used. Further studies are ongoing to deepen the knowledge about the molecular components responsible for the antimicrobial activity. 


\title{
Circular economy \& environmental sustainability
}

\section{Industrial modular technologies and sustainable consequences of insect production}

\author{
S. Smetana $1^{*}$, E. Schmitt ${ }^{2}$, S. Ites ${ }^{1}$, S. Toepfl ${ }^{1}$, A. Mathys ${ }^{3}$ and V. Heinz ${ }^{1}$ \\ ${ }^{1}$ German Institute of Food Technologies (DIL e.V.), Quakenbrück, Germany; ${ }^{2}$ Protix, Industriestraat 3, 5107 NC Dongen, \\ the Netherlands; ${ }^{3}$ ETH Zurich, Institute of Food, Nutrition and Health, Laboratory of Sustainable Food Processing, Zurich, \\ Switzerland; s.smetana@dil-ev.de
}

The lack of protein sources in several parts of the world is triggering the search for flexible and sustainable protein production technologies. Modular insect production technologies are recognised as a potential solution. This study is aimed to define the potential of food and feed production and processing technologies transfer for the design of sustainable modular insect production units. It includes the identification of production requirements of modular, transportable and wide range of feeds applicable technology for two insect species (Hermetia illucens and Tenebrio molitor). The design allowed for the economic analysis and life cycle assessment (LCA) of modular waste treatment $H$. illucens technology, further compared to attributional and consequential LCA of industrial-scale production of the same species fed on food industry side streams. The reference environmental impact results of industrial-scale insect production were based on dataset covering nineteen-month period (2015-2017) of $H$. illucens production and processing with measured variables of water use, feed inputs, electricity and heat consumption, production yields from an industrial producer (Protix, Dongen, the Netherlands). Data gathering of insect processing included direct measurements of similar industrial processes in food and feed industry and material flow modelling. The results indicated that if attributional LCA rules are applied (feed is a free waste material with zero negative burden) both industrial and modular technologies are impacting the environment in the range of $38.83-77.67 \mathrm{mPt}$ ( $\mathrm{mPt}-$ millipoint, $1 \mathrm{kPt}$ is annual impact of one European person, IMPACT2002+ Methodology) with each tonne of waste material treated. If substitution of conventional waste treatment technologies (composting, anaerobic digestion) is considered (consequential LCA approach) then industrial insect production results in positive environmental impact in the range of - 155.34 to $-233 \mathrm{mPt}$ for each tonne of treated material. Modular waste treatment insect technologies were depended on the type of waste material treated and reached impacts from $0.49 \mathrm{mPt}$ to $-199.22 \mathrm{mPt}$ for tonne of waste treated. Both approaches were financially viable, especially if resulted products are sold for minimal prices. Therefore, modular insect production technologies are economically and environmentally viable option for the treatment of some waste types with some insect species (H. illucens). Further research for variations in insect species, production technologies and feed materials are required. 


\title{
Sustainable insect value chains through integration of artificial intelligence algorithms
}

\author{
S. Smetana ${ }^{1 "}$, A. Tonda ${ }^{2}$ and A. Mathys ${ }^{3}$ \\ ${ }^{1}$ German Institute of Food Technologies (DIL e.V.), Quakenbrück, Germany; ${ }^{2}$ UMR 518 MIA, INRAE, Université Paris- \\ Saclay, France; ${ }^{3}$ ETH Zurich, Institute of Food, Nutrition and Health, Laboratory of Sustainable Food Processing, Zurich, \\ Switzerland; s.smetana@dil-ev.de
}

Insect value chains evolve in Europe are moving from pilot trials to large-scale industrial production systems. Such a shift, however, is facing challenges in optimising efficiency and manage potential risks. SUSINCHAIN, a Horizon 2020 Europeanfunded project started in 2019, aims to define the leverages and solutions for the development of sustainable insect value chains, for both food and feed products. In order to assure the sustainability of insect production and consumption it is necessary to define the optimal conditions from multiple perspectives: economic, environmental, safety, nutritional, etc. Such perspectives have different weights at various stages of the value chain. Aforementioned properties are often in contradiction to each other (e.g. increasing economic value versus maximising safety or reducing environmental impact). Currently life cycle assessment (LCA) is the standard for the assessment of environmental impact, allowing experts to take into account multiple criteria at the same time. Nevertheless, the application of LCA to emerging insect production systems is not straightforward, mainly because of the lack of data and emerging technological scales. A possible solution to this issue stems from combining multi-objective optimisation algorithms (MOA) with modular LCA approaches. In order to reflect the variety of parameters and conditions of production and processing, value chains are represented as a combination of modules from feed and food industries. Each module is characterised by inputs and outputs which define its functional value. Therefore, the importance of different inputs and outputs (properties of subsystems) is defined for each module or agglomeration of modules. Once the important features are defined the MOA can be applied to define the optimal value chains, which in turn make it possible to satisfy the needs of different actors in the chain. To compensate for the current lack of data, it could be possible to use machine learning technique to adapt modules from similar systems (feed and food production and processing) to the specific case study of insect production. In a first step, the different criteria that will be considered by the proposed approach for MOA are input parameters: feed (protein, fat content, carbs, etc.), utilities (energy, water, air, chemicals, etc.); and output parameters: yield and frass (relevant nutritional or technical properties) and environmental impacts (direct emissions into air, water and land). Moreover, all the inputs and outputs include associated functions of cost/price and indirect environmental impacts.

\section{Industry perspective on insect sustainability assessment and guidance for the industry}

\author{
E. Schmitt ${ }^{1 *}$, S. Smetana ${ }^{2}$ and A. Mathys ${ }^{3}$ \\ ${ }^{1}$ Protix B.V., Industriestraat 3, 5107 NC Dongen, the Netherlands; ${ }^{2}$ German Institute of Food technologies DIL, Prof.- \\ von-Klitzing-Str. 7, 49610 Quakenbrück, Germany; ETH, Schmelzbergstrasse 9, LFO E 12.2, 8092 Zurich, Switzerland; \\ eric.schmitt@protix.eu
}

General improvements to insect sustainability performance can be expected from the increase of the technology readiness level and economy of scale, including more advanced players in the industry. The talk will highlight how life cycle assessment based sensitivity analysis is a useful guidance for industrial producers interested in a holistic approach to managing their environmental impact profile. Focus will be on the quantitative environmental impact of shifting to non-utilised side-streams for feed. Insights will be also be shared about how insects for food and feed can fit into national and international programs that attempt a holistic environmental impact reduction. In particular, results will be discussed for how the switch to renewable energy is particularly important for bringing the global warming impact of insect production below the levels realised by nature-based production systems not so strongly dependent on energy use, such as fishmeal and crops. 


\title{
Insect production to close the loop: connecting sustainable livestock and crop production
}

\author{
S.Y. Chia ${ }^{1,2}$, K.Y. Barragan-Fonseca ${ }^{1}$, E. van de Zande $^{1}$, M. Wantulla ${ }^{1}$, C.M. Tanga ${ }^{2}$, D.M. Mwangi ${ }^{3}$, J.J.A. van Loon ${ }^{1}$ \\ and M. Dicke ${ }^{1^{*}}$ \\ ${ }^{1}$ Laboratory of Entomology, Wageningen University, P.O. Box 16, 6700 AA Wageningen, the Netherlands; ${ }^{2}$ International \\ Centre of Insect Physiology and Ecology (icipe), P.O. Box 30772-00100, Nairobi, Kenya; ${ }^{3}$ Non-Ruminant Research Institute, \\ Kenya Agricultural and Livestock Research Organization (KALRO), Kakamega 50100, Kenya; marcel.dicke@wur.nl
}

The production of insects for food and feed has much wider implications than only as a sustainable protein source. The production of insects for food and feed has been initiated to provide a high-quality protein source for humans and livestock based on low-quality residual streams as input. This upgrading of low-quality inputs to provide an alternative for soymeal and fishmeal may provide important environmental benefits in terms of mitigation of greenhouse gas emission, reducing land and water use for protein production and biodiversity benefits. The use of insects as feed has important potential to be used by smallholder farmers, who are responsible for the basis of global food production. Because small-scale insect production may be carried out as a low-tech activity it may be readily adopted by smallholder farmers and taken up in rural communities. Moreover, these locally produced insects may be used on-farm to feed livestock such as pigs and poultry while replacing other protein sources such as fishmeal. Thus, the use of insects as feed may contribute to an on-farm circular agriculture that benefits smallholder farmers. Furthermore, the residual streams of insect production, such as frass and insect exuviae, may be valuable input for crop production. These rest streams of insect production may stimulate crop growth and resilience. Here, we will present data on the use of black soldier fly larval meal as replacement of fishmeal in feed for the production of poultry and pigs by smallholder farmers in Kenya. Moreover, we will show the effects of the use of residual streams of the production of three species of insects (house crickets, yellow mealworms and black soldier flies) on crop growth, resilience and interactions with insect herbivores and pollinators in the Netherlands. Our data highlight how the production of insects for food and feed can contribute to a sustainable circular agriculture that connects sustainable livestock and crop production. Moreover, by providing opportunities for smallholder farmers in low and middle income countries, edible insects may provide opportunities to involve smallholder farmers in the low initial capital investments, smallholder insect farmers have good opportunities to increase productivity, improve their livelihood and contribute to food security and a circular economy.

\section{A disruptive technology - insect farming for food and feed in Africa}

\author{
A. Halloran ${ }^{1}$, N. Roos $^{2}$ and D. Verner ${ }^{3 *}$ \\ ${ }^{1}$ Independent Consultant, Copenhagen, Denmark; ${ }^{2}$ University of Copenhagen, Denmark; ${ }^{3}$ The World Bank, 1818 H St, \\ NW, Washington DC 20433, USA; dverner@worldbank.org
}

Insect farming and processing is a nascent industry in Africa, while human consumption of insects in Africa is well documented. This study shows that insect farming is already happening throughout the continent from North to South and East to West Africa and all age groups are participating. This is less known to the outside world. Therefore, the World Bank and the University of Copenhagen have joined forces and launched a country level and a farm level survey to obtain insight assess the insect farming activities occurring throughout Africa. The survey was based on a convenient sampling strategy by reaching out to researchers and insect farmers in 20 different African countries in 2019. Responses were received from a total of 14 countries. At least 14 different insect species are farmed, whereof five species account for $71 \%$ of all farmed insects. The survey identified 849 unique insect farms and estimated the existence of up to 1,790 active farms. Specific farm-level data was gathered from 161 of these farms. In addition, to the insects, some farmers used the biofertiliser produced in the insect production process as fertiliser in their fields. The data reveals that insect feed used on the insect farms mainly originate from organic waste and side streams, such as household or catering waste. Farmers perceive the environmental benefits of insect farming and that fragility and climate change have a negative impact on traditional semi-farmed insect productions. Insect farmers - mainly those producing BSFL for animal feed - recognise the potential positive impacts on the environment through reduced resource use, including water, the effect the flies have in breaking down waste to keep communities clean and safe, and insects use fewer natural resources than livestock. Insect farming has the potential to become a sustainable and effective agricultural production system in Africa. It can contribute to increased food and nutrition security, create climate resilience livelihoods and jobs in the agri-food sector, and increased soil health and general sustainability of the agricultural sector on the continent. As an additional result, an expanding insect sector would increase agri-entrepreneurship and innovation. Women, in particular, would benefit as they oversee feeding the family and assuring meals meet the nutritional needs of children. 


\title{
Learning from a sustainability hot spot analysis applied to the wild harvest cricket sector in Myanmar
}

\author{
D.J. Allan", S.S. Htwe, H. Legge and P.P. Thein \\ ${ }^{1}$ Spectrum, Sustainable Development Knowledge Network, 9C Myaynigone Plaza, Sanchaung Township, Yangon, Myanmar; \\ djallan7@gmail.com
}

Insect eating has been common and popular for a very long time in Myanmar. Little research has been conducted and there is sparse documentation or broader understanding of practices available. Crickets are the most popular and widely consumed of around 50 edible insect species in Myanmar. Current research indicates that there have been significant changes recently: (1) improvements in the technology available for harvesting wild crickets; (2) a move from subsistence to commercial value chains and markets; (3) vastly increased numbers of crickets sold and transported over much greater distances. Very few crickets are farmed in Myanmar and the vast majority is harvested from the wild, raising questions about sustainability as well as food and worker safety. The sustainability hot spot analysis (SHSA) conducted by Spectrum - Sustainable Development Knowledge Network (SDKN) contributes to development in the edible insect sector in Myanmar by increasing understanding of the current wild harvest cricket value chain and identifying which aspects of the value chain phase present potential social and environmental sustainability issues in insect systems. The study uses SHSA to review each phase of the wild cricket value chain from harvesting to consumption and assesses many social and environmental sustainability factors. These include waste emissions, resource consumption, human rights, working conditions and health and safety. In Myanmar and many other countries, wild harvest remains an important sub-sector of the insect industry. Wild harvest sustainability is however threatened due to overharvesting in a context characterised by the absence of information, guidelines, practice guides, regulations and suitable motivators and enablers for farming substitutes. The SHSA guides recommendations for addressing research, legislation and policy gaps in Myanmar, some of which are likely to be applicable to other international locations. It will also help inform gaps in consumer awareness, food safety aspects and worker education. The SHSA has been a lens to help identify several 'hot spots' in the value chain, particularly in harvesting and consumer health and safety. Much more work is needed to address these hot spots and others identified, to help further optimise production systems and improve economic and environmental sustainability. Enhanced policy and practice is needed to ensure conservation and sustainability in line with the precautionary principle.

\section{Sustainable model for agroenergy and feed production through the black soldier fly and microalgae - SMART FEED}

\author{
S. Savoldelli ${ }^{1 *}$, C. Jucker ${ }^{1}$, D. Lupi ${ }^{1}$, S. Colombini ${ }^{2}$, I. Toschi ${ }^{2}$, G. D'Imporzano ${ }^{2,3}$ and F. Adani ${ }^{2}$ \\ ${ }^{1}$ Department of Food, Environmental and Nutritional Sciences, University of Milan, via Celoria 2, 20133 Milan, Italy; \\ ${ }^{2}$ Department of Agricultural and Environmental Sciences, Production, Landscape, Agroenergyg, University of Milan, via \\ Celoria 2, 20133 Milan, Italy; ${ }^{3}$ Consorzio Italbiotec, Via Fantoli 16, 20100 Milan, Italy; sara.savoldelli@unimi.it
}

In the framework of the circular economy, the fly species Hermetia illucens (Diptera Stratiomyidae) occupies an important role as it can grow on a broad spectrum of organic substrates, including wastes, converting them into a high value biomass recapturing nutrients. The waste reduction and the bioconversion ability of the larvae are of particular efficiency and the final biomass (larvae), particularly rich in proteins and lipids, can be exploited for different aims, including meals for livestock, biofuel and biofertiliser. The SMART FEED Project, funded by Fondazione Cariplo, intends to develop a local circular economy model promoting the production of larvae of $H$. illucens on the organic fraction of municipal solid waste (OFMSW) to be used as healthy feed for poultry rearing. In addition, another important source of protein, the microalgae, will be produced on the liquid fraction of the digestate to increase the quality, the sustainability and the nutritional value of the insect biomass. Microalgae has been identified as valuable nutritional dietary supplement for animal nutrition, due to the high protein content, high quality of amino acids, vitamins, antioxidants and other substances beneficial for animal health. Thus, a functional and promising feed, which includes larvae and microalgae, will be formulated and tested as a meal for broiler. Anaerobic digestion (AD) of residual wastes can provide the requested energy for the process. The waste loop will be closed by producing fertilisers to be used in a modern agriculture promoting the use of renewable fertilisers by precision farming. This holistic and multidisciplinary approach will allow to deepen the numerous aspects of the whole process. Different work packages will include: (1) characterization of the OFMSW with particular reference to the biological value of the organic components; (2) insect and microalgae production from the OFMSW and their characterization; (3) production of the SMART-feed, assessment of its quality and safety, in vitro and in vivo digestibility for broiler; (4) Production and evaluation of biogas and fertiliser from the residual OFMSW; (5) assessment of the environmental impact of the circular model; and (6) economic evaluation and estimation of the impact of the SMART-Feed on the regional economy. Particular attention will be turn on the feed quality, safety and the compliance of the final feed. 


\section{Quality control, legislation \& policy}

\section{Identifying Australians barriers to accepting edible insects as an emerging protein source}

\section{Hopkins, J. Danaher, A. Farahnaky, H. Gill and L.P. Newman}

School of Science, College of Science, Engineering and Health, RMIT University, Bundoora West Campus, Victoria, 3083, Australia; S3744095@student.rmit.edu.au

Increasing global populations and the limitations on finite resources will place greater pressure on an already strained food system and of particular concern is protein. Insects offer a nutritious and sustainable protein source, yet despite a rich history, Australians have been hesitant in adopting the practice. This study aimed to identify barriers and explore possible factors that may motivate Australians to accept edible insects as a new source of protein. Participants ( $\mathrm{n}=601 ; 76.2 \%$ female, $23.8 \%$ male; aged 18 -35 years $40.8 \%$, 36-54 years 43.6\%, > 55 years 15.6\%) completed an online survey investigating Australian consumers' barriers to eating insects and what potential motivating factors may overcome these obstacles. Results indicated participants cited 'lack of opportunity' (56.3\%) and 'disgust' (17.7\%) as the main barriers to previously eating insects. The outcomes of this study indicate that by providing Australians with an increased opportunity and improved accessibility to insect-based products, it may be possible that a higher proportion of Australians would be accepting of eating insect-based products. Consumer focused food product and process innovation for developing new appealing food products containing insect ingredients is required as this may improve the palatability and acceptance of edible insects as a protein source. 


\title{
Clarifying regulatory and safety landscape of insects as food and feed in North America
}

\author{
C. Preyer ${ }^{1 *}$, K. Driver ${ }^{2}$ and G. Mott ${ }^{3}$ \\ ${ }^{1}$ Preyer Consulting, LLC, Brookview Drive, Saint Louis, MO 63126, USA; ${ }^{2}$ Enterra Feed, Maple Ridge, BC Canada; ${ }^{3}$ Aspire \\ Food Group, 6231 E Stassney Ln, Austin, TX 78744, USA; All authors represent the North American Coalition for Insect \\ Agriculture (NACIA); cheryl.preyer@gmail.com
}

As the association that brings together Industry Players with interests in North America, The North American Coalition for Insect Agriculture (NACIA) provides a high-level overview of its Strategic Plan. Highlighted is an update on efforts to provide a consolidated safety standard for North America. This document, developed in partnership with agencies in Canada and the United States, will provide clarity to the Insects as Food and Feed Industry throughout North America. While Insects produced as Food or Feed in North America must adhere to their home countries' manufacturing safety requirements, the industry and regulators have been lacking comprehensive standards specific to the Insect Agriculture Industry. This resource closes the gap providing clear direction for industry members as well as regulatory bodies throughout North America.

\section{Entomophagy and the law: challenges and opportunities of the regulatory framework in the EU and around the world}

\author{
N. Carbonnelle \\ Counsel at Bird E Bird LLP, Brussels, Belgium; nicolas.carbonnelle@twobirds.com
}

Human consumption of insects is commonplace in many parts of the world, but is relatively innovative and disruptive in others, including Europe and North America. The introduction of insects in the diet of the average consumer poses legal questions regarding the way insects and the ingredients derived from insects should be regulated. From production rules, including the definition of the substrates that can be used to grow insects intended for human consumption, to food information and safety, through hygiene rules, the insects production and supply chain is a completely new territory for most Western regulators. In the European Union, a detailed set of regulations has classified edible insects in the category of novel foods, which entails that insects intended for human consumption may not be placed on the market without being granted a marketing authorisation first. A thorough safety assessment, centralised at EU level, forms integral part of the approval process. Once approved by the European Commission, a product may be placed on all EU markets. This authorisation scheme is accompanied with a regulatory data protection regime that grants market exclusivity to the holders of authorisation under certain conditions, thereby structuring the emerging market of edible insects in the EU and impacting its organic growth. This presentation compares and puts into perspective the regulations applicable to edible insects around the world, to the purpose of devising what a global level playing field could look like for insect-based foods. 


\section{Authors index}

A

Aartsma, Y.

Adani, F.

Agbodzavu, K.M.

Al Bitar, L.

Allan, D.J.

Ameixa, O.M.C.C.

Andere, A.A.

Antarest, E.

Anyega, A.

Arabzadeh, G.

Ashour, M.

Asimaki, A.

Athanassiou, C.G.

Auger, L.

Azzollini, D.

B

Baldacchino, F.

Barragán-Fonseca, K.B.

Barragán-Fonseca, K.Y.

Bassi, A.

Bastiaens, L.

Beaulieu, A.D.

Beesigamukama, D.

Bellezza Oddon, S.

Belluco, S.

Benning, R.

Bertram, S.M.

Beukeboom, L.W.

Biasato, I.

Bindelle, J.

Binego, L.

Bischel, $\mathrm{H}$.

Boatta, F.

Boeck, G.

Bolhuis, J.E.

Bonelli, M.

Bonilauri, P.

Bonizzi, L.

Borgemeister, C.

Böschen, V.

Bouslama, S.

Brilli, M.

Bruder, T.

Brugiapaglia, A.

Bruno, D.

Buchanan, F.

Buckley, C.A.

Bukamba Tshanga, C.

Bußler, S.

C

Caccia, S.

Cai, M.
Caimi, C.

18,53

Calado, R.

Caligiani, A.

70

19

Caparros Megido, R.

$31,36,45$

Carbonnelle, N.

76

Cardoso, S.F.S.

36,37

Casartelli, M.

Cavini Moreno, A.C.

$27,28,35,41$

Charbonneau, A.R.L.

Chemello, G.

18,53

Chemurot, M.

51

Cheseto, X.

Chia, S.Y.

6

73

52

24,30

Chulu, M.C.

39,62

Claeys, J.

Colombini, S.

74

Conconi, A.M.

Cos, P.

Coudron, C.L.

Couture, M.

Crespo, P.V.

Curatolo, M.

Czekała, W.

\section{D}

18, 53

Dabbou, S.

18

Danaher, J. $\quad 75$

Debaste, F. $\quad 17$

De Boer, I.J.M. $\quad 63,66$

Defilippo, F. 16

18,21

69

28,69

39,62

$27,28,33,40,69$

9,41

21,23

$27,28,35,41$

16

61

42

22

28

28

41

53

Delisle-Houde, M.

Derôme, N.

Deruytter, D.

Deschamps, M.H.

De Smet, J.

Dias, J.

Dicke, M.

Dijkslag, A.

Dimitrova, S.

Dimopoulos, G.

D'Imporzano, G.

Domingues, M.R.

Dorais, M.

Dortmans, B.M.A.

Dottori, M.

Doyen, A.

Dridi, C.

Driver, K.

Durek, J.

Dürr, J.

E

Egger, J.

20,35

Egonyu, J.P.

20, 51 
Egonyu, P.J.

Ekesi, S.

Ekonomou, G.

Ellers, J.

Emery, V.J.

Erikkson, T.

Ermolaev, E.

\section{F}

Fanter, M.

Farahnaky, A.

Fauconnier, M.L.

Fernandez-Bayo, J.D.

Ferretti, E.

Fiaboe, K.K.M.

Fiorilla, E.

Floto-Stammen, S.

Flutsch, G.

Fogliano, V.

Fombong, F.T.

Fortin, A.

Fountoulaki, E.

Fowles, T.

Francis, F.

Francuski, L.

Frooninckx, L.

\section{G}

Gai, F.

Galaverna, G.

Gallen, C.

Gariglio, M.

Gasco, L.

Gedrovica, I.

Gerrits, W.J.J.

Gianfranceschi, N.

Gianotten, N.

Gicheha, M.

Gigante, P.

Gill, H.

Gold, M.

Gono, A.B.

Goossens, S.

Gorrens, E.

Gravel, A.

Grebenteuch, S.

Guidou, C.

\section{H}

Haase, $\mathrm{H}$.

Hadj Saadoun, J.

Halloran, A.

Hamba-Tola, Y.

Hamidou Leyo, I.

Heetkamp, M.J.W.

Heinz, V.

Heiska, S.
Hénault-Ethier, L.

33,40

$6,12,19,20,42,67$

Henry, M.

Heuel, $M$.

24

25

52

32

Hlongwane, Z.T.

31

29,40

29

64

Hoc, B.

Hoek-van den Hil, E.F.

14

56

16

65

25

75

27

74

68

$12,42,67$

18

55,56

47

10, 19

10

33

24

43

$31,36,45$

32

39

Hofman, J.

Holtermans, B.

Homska, N.

Hopkins, I.

Hosseini, A.

Htwe, S.S.

Hussain, Z.B.M.

18

71

Ites, $\mathrm{S}$.

J

Janke, J. 55

Jansen, W.

Jowett, B.

25, 26

Józefiak, A.

$23,24,25,26$

Józefiak, D.

$16,61,74$

\section{K}

$18,53,61$

ucker, C.

17

36

13

24, 30

Karapanagiotidis, I.T.

Katz, H.

Keil, C.

Kelemu, S.

Kelstrup, H.C.

Khamis, F.M.

Kidoido, M.

Kierończyk, B.

Kinyuru, J.N.

Kipkoech, C.

Kone, $\mathrm{M}$.

Korhonen, K.

Korir, N.K.

Kouřimská, L.

8

13

6

29,40

42,67

58

$23,24,25,26$

$10,19,48$

48,69

27,40

55

67

59

25

25

13

59

13

Kulow, F.

$\mathrm{Ku}, \mathrm{S} .57$

Kusumawardhani, T.

$31,60,63$

Kuta, J.

$\mathbf{L}$

Lakemond, C.M.M. $\quad 10$

Lalander, C. 64 
Lamaj, F.

Lazzi, C.

Le Gall, P.

Legge, $\mathrm{H}$.

Le Mintier, I.

Lessard, B.

Lesur, L.

Lewis, M.

Lognay, G.

Lopes, J.C.

Loupiac, C.

Luparelli, A.V.

Lupi, D.

\section{M}

$\mathrm{Ma}, \mathrm{S}$.

Mackie, N.

MacMillan, H.A.

Mäki, M.

Malawey, A.S.

Malumba Kamba, P.

Marchand, Y.

Marie, A.

Marnila, P.

Marquis, D.

Mascaretti, A.

Masset, C.

Mateus, T.L.

Mathys, A.

Mayele, D.

Mazurkiewicz, J.

Meijering, J.V.

Meijer, N.P.

Mente, E.

Meyer, A.M.

Mezdour, S.

Mhemdi, $\mathrm{H}$.

Micenková, L.

Mikołajczak, Z.

Mishyna, M.

Mochoge, B.

Monticelli, M.

Morales-Ramos, J.

Mott, G.

Moussa Ousman, Z.

Muafor, F.J.

Muafor, F.M.

Muilu, T.

Munga, L.K.

Munyai, T.C.

Murugu, D.K.

Musyoka, M.W.

Mutisya, M.M.

Mutsakatira, E.T.

Muungo, L.T.

Muzzatti, M.J.

Mwangi, D.M.

\begin{tabular}{|c|c|c|}
\hline 33 & $\mathbf{N}$ & \\
\hline 70 & Nakimbugwe, D. & 20,67 \\
\hline 47,49 & Nansen, C. & 43 \\
\hline 74 & Naranjo-Guevara, N. & 55,56 \\
\hline 47 & Naser el Deen, S. & 33 \\
\hline 50 & Ndiritu, A.K. & 12 \\
\hline 21 & Neviani, E. & 70 \\
\hline 11 & Newman, L.P. & 75 \\
\hline 31 & Nguyen, T.T.A. & 69 \\
\hline 36,37 & Niassy, S. & 19 \\
\hline 11 & Normandin, É. & 33 \\
\hline 70 & Nsase, $M$. & 49 \\
\hline $16,61,74$ & Numbi Muya, G. & 36 \\
\hline & Nyingi, D.W. & 20 \\
\hline & Nzuma, J. & 58 \\
\hline 44 & & \\
\hline 21 & $\mathbf{O}$ & \\
\hline 30 & Ochoa-Sanabria, C. & 17 \\
\hline 55 & Octavia, M. & 43 \\
\hline 65 & Ojha, S. & 61 \\
\hline 17 & Okello, A. & 58 \\
\hline 21 & Okpara, P.O. & 45 \\
\hline 54 & Onyango, A.N. & 12 \\
\hline 55 & Oonincx, D.G.A.B. & 19,30 \\
\hline 33 & Osuga, I.M. & 12,20 \\
\hline 61 & Otieno, D. & 58 \\
\hline 21 & Oude Lansink, A.G.J.M. & 68 \\
\hline 36,37 & & \\
\hline $7,11,25,27,35,38,43,71,72$ & $\mathbf{P}$ & \\
\hline 17 & Palma, L. & 43 \\
\hline 25,26 & Pantin-Sohier, G. & 54 \\
\hline 68 & Paredes, J.C. & 12 \\
\hline 43 & Parodi, A. & 63,66 \\
\hline 24,30 & Parry, N.J. & 42 \\
\hline 14 & Paul, A. & 27 \\
\hline $11,14,47,49$ & Peguero, D.A. & 11 \\
\hline 11,14 & Persaň, T. & 16,44 \\
\hline 44 & Person, S. & 49 \\
\hline $23,24,25,26$ & Picard, C.J. & 29 \\
\hline 10 & Pieterse, E. & 56 \\
\hline 67 & Pinho, M. & 13 \\
\hline 21 & Pino Moreno, J.M. & 51 \\
\hline 40 & Pipan, M. & 18,21 \\
\hline 76 & Ponce-Reyes, R. & 50 \\
\hline 45 & Preyer, C. & 76 \\
\hline 49 & Psarianos, M. & 15 \\
\hline 47 & Psofakis, P. & 24 \\
\hline 55 & Purcaro, G. & 31 \\
\hline 20 & & \\
\hline 52 & $\mathbf{R}$ & \\
\hline 12 & Rahayu, T. & $31,60,63$ \\
\hline 67 & Ramnuth, M. & 47 \\
\hline 19 & Rawski, M. & $23,24,25,26$ \\
\hline 11 & Renna, M. & 18,53 \\
\hline 52 & Ricci, A. & 61,70 \\
\hline 30 & Richards, C.S. & 11 \\
\hline 20,73 & Rodenburg, T.B. & 19 \\
\hline
\end{tabular}


Rodrigues, D.P.

Rojas, G.

Roos, N.

Roslan, A.M.

Rossi, G.

Rumbos, C.I.

Ruseva, K.

\section{S}

Sandrock, C.

Savoldelli, S.

Scheidegger, A.

Schiavone, A.

Schlüter, O.

Schmitt, E.

Sekonya, J.G.

Sengendo, F.

Sevgan, S.

Sforza, S.

Shikha, O.

Shumo, M.

Slotow, R.

Smetana, S.

Soetemans, L.

Sok, J.

Soomro, A.A.

Sotolu, A.O.

Subramanian, S.

Sule, S.O.

Sypniewski, J.

\section{T}

Tanga, C.M.

Tanga, M.C.

Taoukis, P.

Tarlton, J.

Teerlinck, S.

Terranova, M.

Tettamanti, G.

Thein, P.P.

Thompson, $\mathrm{T}$.

Thu Thu Aung, M.

Tikilili, P.V.

Toepfl, S.

Tomberlin, J.K.

Tonda, A.

Torgerson, K.L.

Torto, B.

Toschi, I.

Tourancheau, M.

Trespeuch, C.

Tweddell, R.J.

Tweedy, D.

Twendi, T.

U

Unger, K.
48,73

65

61

24, 30

56

25

$16,61,74$

35

18,53

15,61

$27,35,71,72$

46

51

6

70

15

42

52

71,72

29

68

38

22

20, 42, 51, 67

22

23

$10,12,19,20,42,51,67,69,73$

6

15

21

39,62

25

$27,28,35,41$

74

6

48,58

4, 38, 44

72

68

6

74

33

21,23

69

40

49

54
Ur Rehman, K.

V

Välimaa, A.L.

55

Van Campenhout, L.

$5,9,41$

Van de Minkelis, D.

10

Vandenberg, G.W.

Vanden Broeck

Van der Fels-Klerx, H.J.

$27,28,33,40,69$

10

14,43

Vandeweyer, D.

Van de Zande, E.

41

73

Van Dijk, K.

Van Engeland, C.

63

17

3, 42

VanLaerhoven, S.L.

Van Loon, J.J.A. 14, 19, 20, 50, 63, 66, 73

Van Miert, S.

Van Moll, L.

Van Schelt, J.

39

Van Zanten, H.H.E.

63, 66

Vašíčková, J.

63, 66

Vaxelaire, $M$.

16,44

Vázquez, J.A.

47, 49

Veldkamp, $\mathrm{T}$.

13

Verner, D.

19,43

Verrastro, V.

Verschoor, G.

Vilcinskas, A.

Vinnerås, B.

Vreugdenhil, C.

Vrontaki, M.

50

5

64

19

30

W

Wachowiak, R. $\quad 25$

Wan, N. $\quad 21$

Wantulla, M. $\quad 73$

Weldon, C.W. $\quad 42$

Wiklicky, V. 64

Wuyts, A. 39

Wynberg, R.P. $\quad 46$

X

Xiao, X.

38

Y

Yakubu, O.S.

Yang, C. $\quad 44$

Yoon, J.

Yu, Z. 38, 44

57

Z

Zhang, J.

38,44

Zheng, L.

38,44

Zimčík, D.

44

Zorrilla, M.J.

65,68

Zurbrügg, C. 


\section{Scientific programme}

\section{KEYNOTES}

Progress in using insects for food and feed

A. van Huis

From research to economics: potential paths forward in the insects as food and feed industry

J.K. Tomberlin

Growing a better future for our industry by harvesting the painful lessons of our past

M. Ashour

Fermentation technology in the insect industry

L. Van Campenhout

Immunity of farmed insects: basic and applied aspects

A. Vilcinskas

Insects as food and feed source: research trends in Africa

M.C. Tanga, X. Cheseto, P.J. Egonyu, S. Kelemu, B. Torto, S. Sevgan and S. Ekesi

Market size for insects as feed

$\mathrm{T}$. Thompson

Is there an opportunity for insects in mainstream commerce? Market size and consumer attitudes towards insects in pet foods

B. Jowett

Holistic sustainability assessment of global food systems - challenges, needs and tools with perspective on insects

A. Mathys

Insights into IPIFF activities and EU policy environment for the insect sector H. Katz

\section{INSECT AS FOOD \& FEED/PROCESSING, CONSERVATION \& FOOD SAFETY OF INSECT PRODUCTS}

The potential of dominant micro-organisms isolated from the gut of the larvae of Hermetia illucens for microbiological control 9 J. De Smet, E. Gorrens, L. Van Moll, P. Cos and L. Van Campenhout

Influence of processing methods on the techno-functional properties of flours from three edible insects

F.T. Fombong, C.M. Tanga, J. Vanden Broeck and J. Kinyuru

Insect processing and insect anatomy

C.M.M. Lakemond, D. Azzollini, D. van de Minkelis, M. Mishyna and V. Fogliano

Thermo-mechanical and pulsed electric field processing effects on protein quality of insect flours

S. Mezdour, C. Loupiac and H. Mhemdi

Faecal sludge management using black soldier fly larvae and product safety

D.A. Peguero, E.T. Mutsakatira, P.V. Tikilili, M. Lewis, C.S. Richards, C.A. Buckley and A. Mathys

Fat quality and phytochemical composition of reared cricket species in Kenya

D.K. Murugu, A.N. Onyango, A.K. Ndiritu, C.M. Tanga, I.M. Osuga, K.K.M. Fiaboe and S. Ekesi 
Development of microbiome tools to improve health and safety of edible insects as components of feeds for livestock C.M. Tanga, A. Anyega, Y. Hamba-Tola and J.C. Paredes

Expired fish feed as a source of $n-3$ fatty acids to modulate the lipid profile of Hermetia illucens

D.P. Rodrigues, M. Pinho, M.R. Domingues, J.A. Vázquez, R. Calado and O.M.C.C. Ameixa

Systematic studies on the antioxidant capacity of yellow mealworm larvae (Tenebrio molitor)

N. Kröncke, C. Keil, F. Kulow, S. Grebenteuch, C. Kanzler, G. Boeck, R. Benning and H. Haase

Extraction of intracellular compounds from Tenebrio molitor by mechanical pressing and pulsed electric field treatments

H. Mhemdi, C. Dridi and S. Mezdour

Review of edible insects metabolising mycotoxins as a safe and sustainable feed/food source

A.M. Meyer, H.J. van der Fels-Klerx, E.F. Hoek-van den Hil and J.J.A. van Loon

Identification and characterisation of yellow mealworm's main proteins in relation to their functional properties

A. Gravel, M. Couture and A. Doyen

Pulsed electric field assisted extraction of valuable compounds from house crickets (Acheta domesticus)

M. Psarianos, G. Dimopoulos, S. Bußler, A.C. Cavini Moreno, P. Taoukis, O. Shikha and O. Schlüter

Bioaccumulation of cadmium in Tenebrio molitor, Zophobas morio and Hermetia illucens

C. Jucker, F. Defilippo, D. Lupi, P. Gigante, S. Savoldelli, E. Ferretti, M. Curatolo, A. Bassi, P. Bonilauri and M. Dottori

Safety aspects of using black soldier fly larvae as feed and food: the study of the bioaccumulation of metals

T. Persaň, J. Kuta, J. Hofman and J. Vašíčková

Experimental study of drying characteristics of Cirina forda caterpillar

C. Bukamba Tshanga, P. Malumba Kamba, C. Van Engeland, D. Mayele, B. Kambashi, J. Bindelle and

F. Debaste

Utilisation of ergot-infected feed by yellow mealworm (Tenebrio molitor) larvae

A.D. Beaulieu, C. Ochoa-Sanabria and F. Buchanan

\section{INSECT AS FEED/ALTERNATIVE PROTEIN SOURCES \& DIET FORMULATIONS}

Live insect larvae for laying hens: effects on egg quality, feather conditions and animal behaviour

S. Bellezza Oddon, I. Biasato, A. Imarisio, M. Pipan, D. Dekleva, S. Dabbou, M. Renna, G. Chemello, M. Gariglio, C. Caimi, E. Fiorilla, L. Gasco and A. Schiavone

Broilers, black soldier fly larvae and behaviour

D.G.A.B. Oonincx, C. Vreugdenhil, T. Veldkamp, V. Fogliano, A. Dijkslag, J.J.A. van Loon and

T.B. Rodenburg

Growth performance, carcass traits and profitability of black soldier fly larvae and Desmodium diets in broiler chicken M.M. Mutisya, J.N. Kinyuru, K.M. Agbodzavu, C. Tanga, M. Gicheha, S. Ekesi and S. Niassy

Full fat black soldier fly larvae meal diets enhances egg production and profitability of smallholder farmers in Kenya

C.M. Tanga, D.W. Nyingi, I.M. Osuga, L.K. Munga, S. Subramanian, J.P. Egonyu, D.M. Mwangi,

D. Nakimbugwe, J.J.A. van Loon, M. Dicke and S. Ekesi

Post-processing and marketing of black soldier fly conversion products - the case of East Java 
Assessing the impact of live black soldier fly larvae supplementation in laying hen diets

D. Dekleva, A.R.L. Charbonneau, P.V. Crespo, N. Wan, M. Monticelli, N. Mackie, J. Tarlton and M. Pipan

Black soldier fly meal inclusion in rainbow trout diets: effects on growth performances from juvenile to portion-size C. Guidou, C. Trespeuch, J. Dias, L. Lesur, C. Masset and Y. Marchand

Apparent digestibility coefficient of cockroach (Periplanata americana) meal by Clarias gariepinus S.O. Sule, A.O. Sotolu and O.S. Yakubu

Different production pathways of insect meal and the effects in feed formulations V. Böschen

The effect of soybean oil replacement by Hermetia illucens fat on young turkey performance and nutrient digestibility J. Sypniewski, Z. Mikołajczak, B. Kierończyk, M. Rawski, W. Czekała and D. Józefiak

Partially defatted black soldier fly meal inclusion in juvenile rainbow trout diets: effects on growth performances C. Guidou, C. Trespeuch and J. Dias

Evaluation of Zophobas morio larvae meal as fishmeal replacer for gilthead seabream diet A. Asimaki, P. Psofakis, G. Ekonomou, E. Mente, C.I. Rumbos, C.G. Athanassiou, E. Fountoulaki, M. Henry and I.T. Karapanagiotidis

Effect of soybean oil replacement by Hermetia illucens fat on broiler chicken performance and nutrients digestibility Z. Mikołajczak, B. Kierończyk, M. Rawski, W. Czekała and D. Józefiak

Black soldier fly larvae as a substitute for soybean in the diets of laying hens

M. Heuel, C. Sandrock, A. Mathys, M. Gold, C. Zurbrügg, M. Kreuzer and M. Terranova

A preliminary study on insect full-fat meals preferences in guppy (Poecilia reticulata)

M. Rawski, J. Kowalska, N. Homska, Z. Mikołajczak, J. Mazurkiewicz, A. Józefiak, B. Kierończyk,

R. Wachowiak and D. Józefiak

How do hydrolyzed insect meals affect sea trout (Salmo trutta trutta) microbiota?

Z. Mikołajczak, M. Rawski, J. Mazurkiewicz, A. Józefiak, B. Kierończyk and D. Józefiak

\section{INSECT PRODUCTION SYSTEMS/GENETICS, NUTRITION, PHYSIOLOGY, HEALTH \& ETHICS OF EDIBLE INSECTS}

Gastrointestinal evacuation in black soldier fly larvae

A. Hosseini, M. Kone, M. Gold, A. Paul, D. Bruno, M. Bonelli, G. Tettamanti, M. Casartelli, E. Schmitt,

A. Mathys, G. Vandenberg and M.H. Deschamps

How the absence of microbiota in black soldier fly (Hermetia illucens) larvae affects gene expression

L. Auger, M.H. Deschamps, S. Bouslama, G. Vandenberg and N. Derome

Morphofunctional adaptation of Hermetia illucens larval midgut to rearing substrates with different nutritional quality

D. Bruno, M. Bonelli, M. Brilli, N. Gianfranceschi, S. Caccia, M. Casartelli and G. Tettamanti

Growth, performance and larval composition of Alphitobius diaperinus on agri-food side-streams

N. Gianotten, L. Soetemans and L. Bastiaens

The yellow mealworm genome: a resource for all

C.J. Picard, T. Erikkson, A.A. Andere, H. Kelstrup and V.J. Emery

Evaluation of the suitability of Greek agricultural by-products for the rearing of Tenebrio molitor

C.I. Rumbos, D.G.A.B. Oonincx, M. Vrontaki, A. Asimaki, E. Mente, I.T. Karapanagiotidis and

C.G. Athanassiou 
Farming fecund crickets: fruitful female fertility after feeding crickets royal jelly

M.J. Muzzatti, H.A. MacMillan and S.M. Bertram

Deuterium labelling of fatty acids in Hermetia illucens larvae

B. Hoc, M.L. Fauconnier, G. Purcaro, G. Lognay, F. Francis and R. Caparros Megido

Effect of black soldier fly density in oviposition cages on egg production

T. Kusumawardhani, T. Rahayu, B.M.A. Dortmans and C. Zurbrügg

Effect of temperature on life-history parameters and production performances of the common housefly, Musca domestica

L. Francuski, W. Jansen and L.W. Beukeboom

Genetic variation in qualitative/quantitative lipid content of three strains of housefly larvae at different temperatures

F. Boatta, W. Jansen, L.W. Beukeboom and J. Ellers

Fecundity and larval growth of Tenebrio molitor reared on differently composed diets of similar nutritional composition S. Naser el Deen, V. Verrastro, F. Lamaj, L. al Bitar and F. Baldacchino

Yeasts as a supplement in diets diets fed to mealworms (Tenebrio molitor)

L. Hénault-Ethier, A. Fortin, D. Marquis, É. Normandin, M. Tourancheau, M.H. Deschamps and

G.W. Vandenberg

Nutritional composition and quality of dried earthworm (Lumbricidae) powder

I. Gedrovica

Black soldier fly (Hermetia illucens): potential and challenges of genetic improvement of insects as food and feed

S. Dimitrova

Preliminary in vitro model of the black soldier fly (Hermetia illucens) larvae midgut

M. Gold, J. Egger, A. Scheidegger, C. Zurbrügg, D. Bruno, M. Bonelli, G. Tettamanti, M. Casartelli,

E. Schmitt and A. Mathys

Insects as sustainable feed for a circular economy: interdisciplinary approach to value chain development

Y. Aartsma and M. Dicke on behalf of the INSECTFEED consortium

Alternative diets and their effect on the growth of Zophobas morio larvae

S.F.S. Cardoso, T.L. Mateus and J.C. Lopes

The edible caterpillar Mikombidila from the west Democratic Republic of Congo: description, life cycle and breeding

G. Numbi Muya, R. Caparros Megido, F. Francis and B. Kambashi Mutiaka

Alternative diets and their effect on the growth of Tenebrio molitor larvae

S.F.S. Cardoso, T.L. Mateus and J.C. Lopes

\section{INSECT PRODUCTION SYSTEMS/PROCESSING ORGANIC SIDE STREAMS \& WASTE MANAGEMENT}

Impact of different gut bacteria on soybean curd residue co-conversion by black soldier fly larvae

A.A. Soomro, M. Cai, K. ur Rehman, L. Zheng, X. Xiao, A. Mathys, M. Gold, J.K. Tomberlin, Z. Yu and

J. Zhang

Physical characterisation of black soldier fly rearing diets

L. Frooninckx, S. Goossens, A. Wuyts and S. Van Miert

Does juice make them fat - processing grass juice with black soldier fly larvae (Hermetia illucens)

C.L. Coudron, D. Deruytter, J. Claeys and S. Teerlinck 
Maturity and fertilising potential of black soldier fly manure (frass) fed residual organic matter from grocery stores

L. Hénault-Ethier, M. Kone, M.H. Deschamps and G.W. Vandenberg

Maximising mycotoxin bioremediation of agriculture wastes: mealworms and dried distillers grains with solubles H.C. Kelstrup, D. Tweedy, J. Morales-Ramos, G. Rojas and V.J. Emery

Mite infestation and its risk assessment to a black soldier fly colony: identification, management and solutions T. Bruder

Black soldier fly larvae rearing on side streams: impact on bacterial biota in different gut regions and larval growth

D. Vandeweyer, D. Bruno, J. De Smet, M. Bonelli, M. Casartelli, G. Tettamanti and L. Van Campenhout

Influence of temperature on selected life-history traits of black soldier fly (Hermetia illucens) reared on two common urban organic waste streams in Kenya

M. Shumo, F.M. Khamis, C.M. Tanga, K.K.M. Fiaboe, S. Subramanian, S. Ekesi, A. van Huis and

C. Borgemeister

Effect of including chicken manure with pre-consumer waste on black soldier fly larval performance and bioconversion N.J. Parry and C.W. Weldon

The influence of residue microbes on black soldier fly larvae (Hermetia illucens) treatment of two agricultural byproducts M. Gold, T. Fowles, J.D. Fernandez-Bayo, L. Palma, C. Zurbrügg, C. Nansen, H. Bischel and A. Mathys

Introduction to the EU H2020 project SUSINCHAIN (SUStainable INsect CHAIN)

T. Veldkamp, H.J. van der Fels-Klerx, N.P. Meijer and M. Octavia

Degradation and proposed pathway of ciprofloxacin by black soldier fly larvae combined with its intestinal microbes C. Yang, S. Ma, M. Cai, L. Zheng, J.K. Tomberlin, Z. Yu and J. Zhang

Dynamics of the occurrence of the ARGs during cattle manure processing by Hermetia illucens J. Vašíčková, D. Zimčík, L. Micenková and T. Persaň

The role of the black soldier fly, Hermetia illucens in combating municipal waste in Windsor-Essex P.O. Okpara and S.L. VanLaerhoven

Chemical composition of Musca domestica maggots fed different agricultural substrates from ther region Niamey, Niger I. Hamidou Leyo, Z. Moussa Ousman, F. Francis and R. Caparros Megido

\section{HARVESTING \& TRADITIONAL ROLE OF EATING INSECTS/SUSTAINABLE CONSUMPTION \& FOOD SECURITY}

Exploring the governance of an edible insect trade chain through the lens of mopane worms in southern Africa

J.G. Sekonya and R.P. Wynberg

Communities, insects and sustainable food production systems - experiences of insect gatherers in Uganda

L. Binego

Promoting the production and transformation of edible insects in West and Central Africa

P. Le Gall, F.M. Muafor, S. Mezdour, I. Le Mintier, G. Flutsch, M. Vaxelaire and M. Ramnuth

Edible insects for child nutrition

C. Kipkoech, J. Kinyuru and N. Roos

Bamboo worm harvesting in Myanmar: more benefits or threats?

M. Thu Thu Aung and J. Dürr 
The market of edible caterpillars in the Republic of Central Africa

M. Vaxelaire, F.J. Muafor, S. Mezdour and P. Le Gall

Edible caterpillars and insects in North Kivu: a potential asset for the development and strengthening of community forestry 49 S. Person, M. Nsase and T. Twendi

Entomophagy in Australia: could insects solve the impending food security challenge?

R. Ponce-Reyes and B. Lessard

Insects for peace: ex-combatants as smallholder insect producers in post-conflict Colombia

K.B. Barragán-Fonseca, K.Y. Barragán-Fonseca, G. Verschoor, J.J.A. van Loon and M. Dicke

Improving traditional insect trapping techniques to enhance crop protection and supply of edible insects in East Africa J.P. Egonyu, F. Sengendo, M. Chemurot, C.M. Tanga and S. Subramanian

Anthropoenthomophagy in Mexico: a review of its history, current situation and perspectives

J.M. Pino Moreno

Diversity and nutritional composition of edible insects in Sub-Saharan Africa

Z.T. Hlongwane, R. Slotow and T.C. Munyai

Nutritional value of edible termites Macrotermes falciger collected from Lusaka District, Zambia, a potential agent against Malnutrition

M.C. Chulu, L.T. Muungo and A.B. Gono

\section{PERCEPTION, MARKETING \& ECONOMICS}

Overall acceptability of fillets of rainbow trout fed diets with increasing levels of Tenebrio molitor larva meal

A. Brugiapaglia, S. Bellezza Oddon, I. Biasato, M. Renna, G. Chemello, C. Caimi, A. Schiavone, F. Gai and L. Gasco

Role of culture in the acceptability of insects as food

A. Marie, G. Pantin-Sohier and C. Gallen

Bugs for the future: a step-by-step education guide for learning about sustainability with insects at home \& in schools K. Unger

Market opportunities for insects in dog food

S. Floto-Stammen, N. Naranjo-Guevara and J. Janke

Potential of increasing the insect production in farms: the case of Northern Ostrobothnia, Finland

K. Korhonen, S. Heiska, P. Marnila, T. Muilu, M. Mäki and A.L. Välimaa

Chicken fed insects must still taste like chicken

L.C. Hoffman and E. Pieterse

Acceptance of Dutch and German students towards insects in feed and food

N. Naranjo-Guevara, S. Floto-Stammen, M. Fanter, A.M. Conconi and K. Ruseva

It's smart and delicious - how to convince Americans to adopt insect protein

J. Yoon

Bugs in schools: universities using insects to valorise food waste

$\mathrm{S}$. Ku 
Understanding farmers' perceived benefits and value-chain governance of insect-based chicken feed in Kenya

A. Okello, J. Nzuma, D. Otieno and M. Kidoido

Online shopping of edible insects in Myanmar

M. Thu Thu Aung and J. Dürr

Factors affecting nutritional value of edible insects and their acceptability

\section{INSECT PRODUCTION SYSTEMS/OPTIMISATION, ENGINEERING, AUTOMATATION \& SECURITY}

Reducing spatial footprint and handling in a black soldier fly bio-waste conversion unit using incubator containers

B.M.A. Dortmans, T. Kusumawardhani, T. Rahayu and C. Zurbrügg

Synchronous front-face fluorescence measurement as a method to rapid discrimination of edible insect powders

G. Rossi, S. Bußler, J. Durek, S. Ojha and O. Schlüter

Developing of low-impact diets for the mass rearing of Acheta domesticus

C. Jucker, S. Belluco, D. Lupi, S. Savoldelli, L. Bonizzi, A. Ricci, A. Mascaretti and L. Gasco

Lost in the desert: influence on wet feed distribution on the density and growth rate of Tenebrio molitor

D. Deruytter, C.L. Coudron, J. Claeys and S. Teerlinck

My way or the highway: transport conditions for Tenebrio molitor

J. Claeys, C.L. Coudron, D. Deruytter and S. Teerlinck

A. Parodi, K. van Dijk, J.J.A. van Loon, I.J.M. de Boer, J. van Schelt and H.H.E. van Zanten

Effect of prepupae density in pupation crates on emergence rate of black soldier flies

T. Rahayu, T. Kusumawardhani, B.M.A. Dortmans and C. Zurbrügg

Optimisation of treatment duration in black soldier fly larvae composting

E. Ermolaev, C. Lalander and B. Vinnerås

Comparison of black soldier fly eclosion success in two environments: the impact of variance in temperature and humidity 64 V. Wiklicky and C. Lalander

New vertical solution in automatic black soldier fly production

B. Holtermans

Optimising black soldier fly breeding in tropical conditions

A.S. Malawey, A.M. Roslan and M.J. Zorrilla

Gaseous emissions during black soldier fly rearing in a mass-rearing diet and pig manure

A. Parodi, I.J.M. de Boer, W.J.J. Gerrits, J.J.A. van Loon, M.J.W. Heetkamp, J. van Schelt, J.E. Bolhuis and

H.H.E. van Zanten

\section{BIOTECHNOLOGY \& NON-FOOD APPLICATIONS OF INSECT}

Effects of black soldier fly frass-fertiliser on the growth, yield and nitrogen use efficiency of maize in Central Kenya

D. Beesigamukama, B. Mochoge, N.K. Korir, D. Nakimbugwe, K.K.M. Fiaboe, F.M. Khamis,

S. Subramanian, M.W. Musyoka, S. Ekesi and C.M. Tanga 
Effect of composted black soldier fly larvae frass on plant growth performance

M.J. Zorrilla and Z.B.M. Hussain

Progression towards circular agriculture - gaining knowledge of insect waste streams as a crop and soil health promoter K.L. Torgerson, J.V. Meijering, J. Sok, M. Dicke and A.G.J.M. Oude Lansink

Fate of organic waste and pesticides in black soldier fly compositing

C. Kipkoech and C.M. Tanga

In vitro antimicrobial activity of frass produced by the black soldier fly larvae against plant pathogens

G. Arabzadeh, T.T.A. Nguyen, M. Delisle-Houde, M. Dorais, M.H. Deschamps, N. Derôme, R.J. Tweddell and G.W. Vandenberg

Search for antimicrobial molecules from lactic acid fermentation of black soldier fly prepupae and related byproducts

A.V. Luparelli, J. Hadj Saadoun, A. Ricci, E. Neviani, G. Galaverna, C. Lazzi, A. Caligiani and S. Sforza

\section{CIRCULAR ECONOMY \& ENVIRONMENTAL SUSTAINABILITY}

Industrial modular technologies and sustainable consequences of insect production

S. Smetana, E. Schmitt, S. Ites, S. Toepfl, A. Mathys and V. Heinz

Sustainable insect value chains through integration of artificial intelligence algorithms

S. Smetana, A. Tonda and A. Mathys

Industry perspective on insect sustainability assessment and guidance for the industry

E. Schmitt, S. Smetana and A. Mathys

Insect production to close the loop: connecting sustainable livestock and crop production

S.Y. Chia, K.Y. Barragan-Fonseca, E. van de Zande, M. Wantulla, C.M. Tanga, D.M. Mwangi,

J.J.A. van Loon and M. Dicke

A disruptive technology - insect farming for food and feed in Africa

A. Halloran, N. Roos and D. Verner

Learning from a sustainability hot spot analysis applied to the wild harvest cricket sector in Myanmar

Sustainable model for agroenergy and feed production through the black soldier fly and microalgae - SMART FEED

S. Savoldelli, C. Jucker, D. Lupi, S. Colombini, I. Toschi, G. D’Imporzano and F. Adani

\section{QUALITY CONTROL, LEGISLATION \& POLICY}

Identifying Australians barriers to accepting edible insects as an emerging protein source

I. Hopkins, J. Danaher, A. Farahnaky, H. Gill and L.P. Newman

Clarifying regulatory and safety landscape of insects as food and feed in North America

C. Preyer, K. Driver and G. Mott

Entomophagy and the law: challenges and opportunities of the regulatory framework in the EU and around the world 